\title{
Rapid Construction of the ABCE Tetracyclic Tertiary Amine Skeleton in Daphenylline Enabled by an Amine-Borane Complexation Strategy
}

\author{
Meng Deng, Yanmin Yao, Xiaohu Li, Nan Li, Xiao Zhang, Guangxin Liang* \\ State Key Laboratory of Elemento-Organic Chemistry, College of Chemistry, Nankai University; \\ Tianjin 300071, China \\ E-mail: Lianggx@nankai.edu.cn.
}

\section{Table of Contents}

I Experimental Procedures and Spectroscopic Data of Compounds (S2-S10)

II $\quad{ }^{1} \mathrm{H}$ and ${ }^{13} \mathrm{C}$ NMR Spectra of Compounds (S11-S24)

III $\quad{ }^{11} B$ NMR Spectra of Compounds (S25-S28)

IV ORTEP Drawings and Crystallographic Data of Compounds (S29-S35) 


\section{Experimental Procedures and Spectroscopic Data of Compounds}

\section{General Information.}

All reactions were carried out under an argon atmosphere with dry solvents under anhydrous conditions, unless otherwise noted. Reagents were purchased at the highest commercial quality and used without further purification. Tetrahydrofuran (THF), hexane, benzene and toluene were distilled from sodium/benzophenone ketyl immediately before use. Methylene chloride $\left(\mathrm{CH}_{2} \mathrm{Cl}_{2}\right)$, acetonitrile $\left(\mathrm{CH}_{3} \mathrm{CN}\right)$ and $\mathrm{N}, \mathrm{N}$-dimethylformamide (DMF) were distilled from calcium hydride and stored under an argon atmosphere. Methanol $(\mathrm{MeOH})$ was distilled form magnesium and stored under an argon atmosphere. Reactions were monitored by thin layer chromatography (TLC) carried out on silica gel GF254 plates using UV light as visualizing agent or basic aqueous potassium permanganate as developing agent. Column chromatography was performed on silica gel (200-300 mesh). ${ }^{1} \mathrm{H}$ and ${ }^{13} \mathrm{C}$ NMR spectra were recorded on Bruker AMX-400 spectrometers (at $400 \mathrm{MHz}$ and $101 \mathrm{MHz}$ respectively) and calibrated by using residual undeuterated chloroform $\left(\delta_{\mathrm{H}}=7.26 \mathrm{ppm}\right)$ and $\mathrm{CDCl}_{3}$ $\left(\delta_{\mathrm{C}}=77.16 \mathrm{ppm}\right)$ as internal references. ${ }^{11} \mathrm{~B}$ NMR spectra was recorded on Bruker AMX-400 spectrometers (at $128 \mathrm{MHz}$ ) and calibrated by an external standard $\left(0.00 \mathrm{ppm}\right.$ for $\left.\mathrm{BF}_{3} \bullet \mathrm{Et}_{2} \mathrm{O}\right)$. The following abbreviations are used to designate multiplicities: $\mathrm{s}=$ singlet, $\mathrm{d}=$ doublet, $\mathrm{t}=$ triplet, $\mathrm{q}=$ quartet, $\mathrm{m}=$ multiplet, $\mathrm{br}=$ broad. IR spectra were recorded on a Bruker 550 spectrometer. Melting points (m.p.) were recorded on a RY-1A apparatus. High-resolution mass spectra (HRMS) were acquired using Varian 7.0T FTMS or Agilent 6520 Q-TOF LC/MS with electrospray ionization (ESI) source.

\section{Experimental Procedures and Spectroscopic Data}

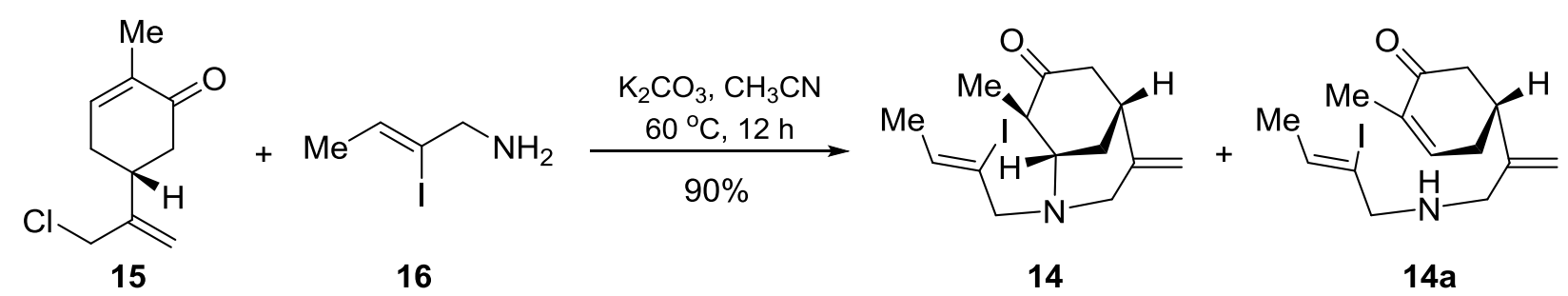

Aza[3.3.1]bicycle 14: To a stirred solution of $15(6.10 \mathrm{~g}, 33.0 \mathrm{mmol})$ in $\mathrm{CH}_{3} \mathrm{CN}(60 \mathrm{~mL})$ was added $16(9.75 \mathrm{~g}, 49.5 \mathrm{mmol})$ and $\mathrm{K}_{2} \mathrm{CO}_{3}(4.56 \mathrm{~g}, 33.0 \mathrm{mmol})$. After stirring at $60{ }^{\circ} \mathrm{C}$ for $12 \mathrm{~h}$, the reaction mixture was concentrated under vacuum and the residue so obtained was soaked thoroughly with hexane $(120 \mathrm{~mL})$. The resulting suspension was filtered and concentrated to give a mixture of $\mathbf{1 4}$ and 14a in a ratio of 7:1 (11.3 g, NMR purity: 90\%) as a light yellow oil, which was directly used in the next step without further purification. When storing the above-mentioned filtrate at $-20{ }^{\circ} \mathrm{C}$ for $12 \mathrm{~h}$, 
the ratio of $\mathbf{1 4}$ and 14a could be improved to over 40:1. Data for 14: $R_{\mathrm{f}}=0.40$ (EtOAc:petroleum ether 1:8, decomposable); $[\mathrm{a}]_{\mathrm{D}}^{16}=+101.7\left(c=1.0, \mathrm{CHCl}_{3}\right) ;{ }^{1} \mathrm{H} \mathrm{NMR}\left(400 \mathrm{MHz}, \mathrm{CDCl}_{3}\right): \delta=5.83(\mathrm{q}$, $J=5.6 \mathrm{~Hz}, 1 \mathrm{H}), 4.82(\mathrm{~s}, 1 \mathrm{H}), 4.63(\mathrm{~s}, 1 \mathrm{H}), 3.42(\mathrm{~d}, J=14.0 \mathrm{~Hz}, 1 \mathrm{H}), 3.27(\mathrm{~d}, J=14.0 \mathrm{~Hz}, 1 \mathrm{H})$, 3.18-3.07 (m, 2 H), 2.97 (s, $1 \mathrm{H}), 2.82(\mathrm{~d}, J=16.0 \mathrm{~Hz}, 1 \mathrm{H}), 2.61-2.55(\mathrm{~m}, 1 \mathrm{H}), 2.50-2.43(\mathrm{~m}, 2 \mathrm{H})$, 2.25-2.19 (m, $1 \mathrm{H}), 1.84-1.80(\mathrm{~m}, 1 \mathrm{H}), 1.79(\mathrm{~d}, J=5.6 \mathrm{~Hz}, 3 \mathrm{H}), 1.29(\mathrm{~d}, J=6.8 \mathrm{~Hz}, 3 \mathrm{H}) \mathrm{ppm} ;{ }^{13} \mathrm{C}$ NMR (101 MHz, $\left.\mathrm{CDCl}_{3}\right): \delta=212.3,145.6,131.4,110.6,110.0,65.6,60.2,50.2,48.9,47.6,40.3$, 31.4, 21.8, 12.4 ppm; IR (thin film): $v_{\max }=2929,1702,1674,1451,1343,1103,1038,969,902,812$, $673 \mathrm{~cm}^{-1}$; HRMS (ESI): Calcd for $\mathrm{C}_{14} \mathrm{H}_{21} \mathrm{NOI}[\mathrm{M}+\mathrm{H}]^{+} 346.0662$, found: 346.0663 . When purified the freshly prepared crude $\mathbf{1 4}$ by flash column chromatography (silic gel), the retro-aza-Michael addition process was enhanced and the ratio of $\mathbf{1 4}$ and $\mathbf{1 4 a}$ could be reduced to ca. $1: 15$. Data for $14 \mathrm{a}: R_{\mathrm{f}}=0.50$ (EtOAc:petroleum ether 1:2, decomposable); $[\mathrm{a}]_{\mathrm{D}}^{12}=-24.5\left(c=1.0, \mathrm{CHCl}_{3}\right) ;{ }^{1} \mathrm{H} \mathrm{NMR}(400 \mathrm{MHz}$, $\left.\mathrm{CDCl}_{3}\right): \delta=6.75(\mathrm{~d}, J=5.8 \mathrm{~Hz}, 1 \mathrm{H}), 5.79(\mathrm{q}, J=6.2 \mathrm{~Hz}, 1 \mathrm{H}), 5.06(\mathrm{~s}, 1 \mathrm{H}), 4.92(\mathrm{~s}, 1 \mathrm{H}), 3.41(\mathrm{~s}, 2$ H), $3.11(\mathrm{~s}, 2 \mathrm{H}), 2.92-2.77(\mathrm{~m}, 1 \mathrm{H}), 2.62(\mathrm{dd}, J=16.0,3.7 \mathrm{~Hz}, 1 \mathrm{H}), 2.54-2.45$ (m, $1 \mathrm{H}), 2.44-2.26$ $(\mathrm{m}, 2 \mathrm{H}), 1.80(\mathrm{~d}, J=6.0 \mathrm{~Hz}, 3 \mathrm{H}), 1.78(\mathrm{~s}, 3 \mathrm{H}), 1.56$ (brs, $1 \mathrm{H}) \mathrm{ppm} ;{ }^{13} \mathrm{C} \mathrm{NMR}\left(101 \mathrm{MHz}, \mathrm{CDCl}_{3}\right)$ : $\delta=199.9,149.2,144.8,135.6,132.0,112.0,111.1,60.4,50.8,43.6,39.3,31.9,21.8,15.8$ ppm; IR (thin film): $v_{\max }=2921,1672,1450,1366,1299,1108,901,802,750 \mathrm{~cm}^{-1}$; HRMS (ESI): Calcd for $\mathrm{C}_{14} \mathrm{H}_{21} \mathrm{NOI}[\mathrm{M}+\mathrm{H}]^{+}$346.0662, found: 346.0663 .

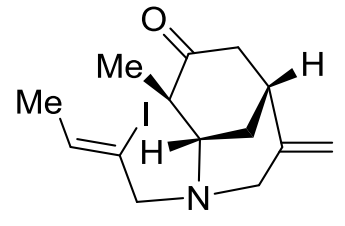

14

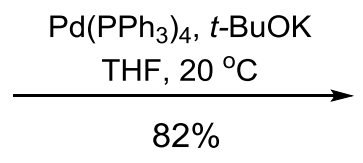

$82 \%$

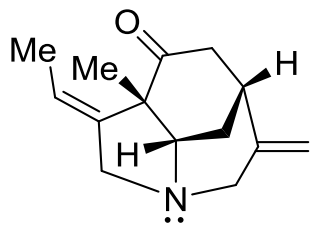

13

Aza-tricyclic ketone 13: To a stirred solution of 14 (5.62 g, $16.3 \mathrm{mmol})$ in THF (160 mL) was added $\mathrm{Pd}\left(\mathrm{PPh}_{3}\right)_{4}(2.83 \mathrm{~g}, 2.44 \mathrm{mmol})$ and $t$-BuOK $(1.92 \mathrm{~g}, 17.1 \mathrm{mmol})$. After stirring at $20{ }^{\circ} \mathrm{C}$ for $24 \mathrm{~h}$, the reaction mixture was quenched with brine $(60 \mathrm{~mL})$ and extracted with $t$-BuOMe $(3 \times 60 \mathrm{~mL})$. The combined organic phases were dried over anhydrous $\mathrm{Na}_{2} \mathrm{SO}_{4}$, filtered, and concentrated under vacuum. The residue so obtained was purified by flash column chromatography with EtOAc/MeOH (40:1 $\rightarrow$ 10:1) to give aza-tricyclic ketone $13(2.90 \mathrm{~g}, 13.3 \mathrm{mmol}, 82 \%)$ as a light yellow oil. Data for 13: $R_{\mathrm{f}}=$ 0.45 (ethyl acetate:methanol 4:1); $[\mathrm{a}]_{\mathrm{D}}^{32}=-287.4\left(c=0.8, \mathrm{CHCl}_{3}\right) ;{ }^{1} \mathrm{H} \mathrm{NMR}\left(400 \mathrm{MHz}, \mathrm{CDCl}_{3}\right): \delta=$ 5.72-5.56 (m, $1 \mathrm{H}), 4.82(\mathrm{~s}, 1 \mathrm{H}), 4.80(\mathrm{~s}, 1 \mathrm{H}), 3.63-3.51(\mathrm{~m}, 1 \mathrm{H}), 3.24(\mathrm{~d}, J=14.1 \mathrm{~Hz}, 1 \mathrm{H}), 3.17-$ $3.04(\mathrm{~m}, 2 \mathrm{H}), 2.91-2.78(\mathrm{~m}, 2 \mathrm{H}), 2.64(\mathrm{dd}, J=17.3,5.7 \mathrm{~Hz}, 1 \mathrm{H}), 2.41(\mathrm{dt}, J=17.3,2.1 \mathrm{~Hz}, 1 \mathrm{H})$, 2.20-2.08 (m, $2 \mathrm{H}), 1.94(\mathrm{dd}, J=7.2,2.3 \mathrm{~Hz}, 3 \mathrm{H}), 1.45$ (s, $3 \mathrm{H}) \mathrm{ppm} ;{ }^{13} \mathrm{C} \mathrm{NMR}\left(101 \mathrm{MHz}, \mathrm{CDCl}_{3}\right)$ : $\delta=212.8,146.4,142.5,122.5,109.6,67.9,61.8,56.4,52.8,45.5,38.0,27.8,21.8,14.9$ ppm; IR (thin 
film): $v_{\max }=2936,2787,1704,1656,1448,1407,1376,1290,1264,1183,1136,1113,1087,1016$, 897, $820 \mathrm{~cm}^{-1}$; HRMS (ESI): Calcd for $\mathrm{C}_{14} \mathrm{H}_{20} \mathrm{NO}[\mathrm{M}+\mathrm{H}]^{+} 218.1539$, found: 218.1542 .

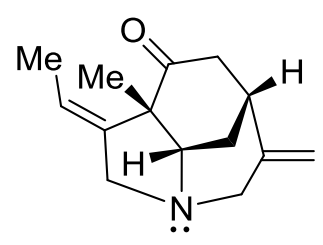

13

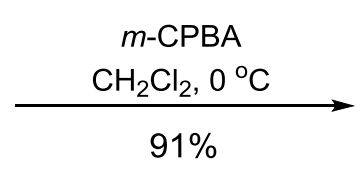

$91 \%$

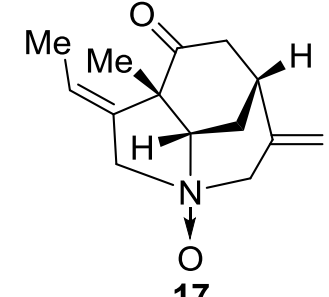

17

The $N$-oxide derivative 17: To a stirred solution of $\mathbf{1 3}(109 \mathrm{mg}, 0.50 \mathrm{mmol})$ in $\mathrm{CH}_{2} \mathrm{Cl}_{2}(10 \mathrm{~mL})$ was added $m$-CPBA (content $70 \%, 123 \mathrm{mg}, 0.50 \mathrm{mmol}$ ). After stirring at $0{ }^{\circ} \mathrm{C}$ for $1 \mathrm{~h}$, the reaction mixture was directly subjected to flash column chromatography using EtOAc/MeOH $(50: 1 \rightarrow 10: 1)$ as eluent to give the $N$-oxide derivative $17(106 \mathrm{mg}, 0.45 \mathrm{mmol}, 91 \%)$ as a light yellow solid. Data for 17: $R_{\mathrm{f}}=$ 0.60 (ethyl acetate:methanol 4:1); m.p. $=107-109{ }^{\circ} \mathrm{C} ;[\mathrm{a}]_{\mathrm{D}}^{33}=-80.5\left(c=0.8\right.$, EtOAc); ${ }^{1} \mathrm{H}$ NMR $(400$ $\left.\mathrm{MHz}, \mathrm{CDCl}_{3}\right): \delta=5.89-5.68(\mathrm{~m}, 1 \mathrm{H}), 5.18(\mathrm{~s}, 1 \mathrm{H}), 5.10(\mathrm{~s}, 1 \mathrm{H}), 4.21-4.07(\mathrm{~m}, 1 \mathrm{H}), 4.02(\mathrm{~d}, J=$ $14.3 \mathrm{~Hz}, 1 \mathrm{H}), 3.75$ (q, $J=14.6 \mathrm{~Hz}, 2 \mathrm{H}), 3.69-3.62$ (m, $1 \mathrm{H}), 3.06-2.98$ (m, $1 \mathrm{H}), 2.86$ (ddd, $J=14.5$, 6.3, 3.2 Hz, $1 \mathrm{H}), 2.75(\mathrm{dd}, J=17.3,5.3 \mathrm{~Hz}, 1 \mathrm{H}), 2.50(\mathrm{dt}, J=17.3,2.5 \mathrm{~Hz}, 1 \mathrm{H}), 2.07(\mathrm{dt}, J=14.5$, $2.7 \mathrm{~Hz}, 1 \mathrm{H}), 2.00(\mathrm{dd}, J=7.3,2.4,3 \mathrm{H}), 1.60$ (s, $3 \mathrm{H}) \mathrm{ppm} ;{ }^{13} \mathrm{C} \mathrm{NMR}\left(101 \mathrm{MHz}, \mathrm{CDCl}_{3}\right): \delta=209.5$, $139.3,131.8,127.1,116.7,80.7,76.6,65.0,56.0,45.3,36.6,23.2,22.4,15.6$ ppm; IR (thin film): $v_{\max }$ $=3420,2943,1708,1680,1655,1444,1101,1042,984,925,794,660 \mathrm{~cm}^{-1}$; HRMS (ESI): Calcd for $\mathrm{C}_{14} \mathrm{H}_{20} \mathrm{NO}_{2}[\mathrm{M}+\mathrm{H}]^{+}$234.1489, found: 234.1493; CCDC 1893589 contains the supplementary crystallographic data for $\mathbf{1 7}$ and is available free of charge from The Cambridge Crystallographic Data Centre via www.ccdc.cam.ac.uk/data_request/cif.

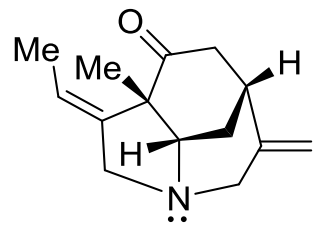

13

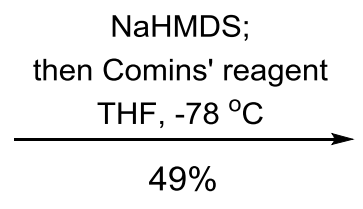

$49 \%$

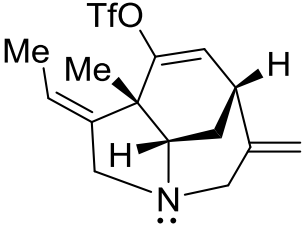

19

Triflate 19: To a stirred solution of $\mathbf{1 3}(217 \mathrm{mg}, 1.0 \mathrm{mmol})$ in THF (20 mL) was slowly added NaHMDS $(0.75 \mathrm{~mL}, 2.0 \mathrm{M}$ in THF, $1.5 \mathrm{mmol})$ at $-78^{\circ} \mathrm{C}$. After stirred at that temperature for $1 \mathrm{~h}$, Comins' reagent $(589 \mathrm{mg}, 1.5 \mathrm{mmol})$ in THF $(5 \mathrm{~mL})$ was added. The resulting mixture was gradually warmed to $0{ }^{\circ} \mathrm{C}$ in $4 \mathrm{~h}$ before it was quenched with brine $(10 \mathrm{~mL})$ and extracted with EtOAc $(3 \times 20$ $\mathrm{mL}$ ). The combined organic phases were dried over anhydrous $\mathrm{Na}_{2} \mathrm{SO}_{4}$, filtered, and concentrated under vacuum. The residue so obtained was purified by flash column chromatography with EtOAc/MeOH $(50: 1 \rightarrow 10: 1)$ to give triflate $19(171 \mathrm{mg}, 0.49 \mathrm{mmol}, 49 \%)$ as a colorless oil. Data for 19: $R_{\mathrm{f}}=0.35$ (ethyl acetate:methanol 10:1); $[\mathrm{a}]_{\mathrm{D}}^{12}=+12.6\left(c=1.0, \mathrm{CHCl}_{3}\right) ;{ }^{1} \mathrm{H} \mathrm{NMR}(400 \mathrm{MHz}$, 
$\left.\mathrm{CDCl}_{3}\right): \delta=5.72(\mathrm{~d}, J=7.1 \mathrm{~Hz}, 1 \mathrm{H}), 5.70-5.61(\mathrm{~m}, 1 \mathrm{H}), 4.89(\mathrm{~s}, 1 \mathrm{H}), 4.88(\mathrm{~s}, 1 \mathrm{H}), 3.70-3.60(\mathrm{~m}$, $1 \mathrm{H}), 3.37$ (d, $J=13.0 \mathrm{~Hz}, 1 \mathrm{H}), 3.29-3.19(\mathrm{~m}, 2 \mathrm{H}), 3.14-3.06(\mathrm{~m}, 2 \mathrm{H}), 2.01-1.94(\mathrm{~m}, 2 \mathrm{H}), 1.84$ (d, $J=6.7 \mathrm{~Hz}, 3 \mathrm{H}), 1.47(\mathrm{~s}, 3 \mathrm{H}) \mathrm{ppm} ;{ }^{13} \mathrm{C} \mathrm{NMR}\left(101 \mathrm{MHz}, \mathrm{CDCl}_{3}\right): \delta=153.4,143.5,142.0,122.7$, $118.5(\mathrm{q}, J=319.7 \mathrm{~Hz}), 118.1,110.0,68.4,62.2,53.1,47.7,37.4,26.7,21.8,14.9 \mathrm{ppm}$; IR (thin film): $v_{\max }=2934,1728,1662,1415,1244,1210,1142,990,894,840,607 \mathrm{~cm}^{-1}$; HRMS (ESI): Calcd for $\mathrm{C}_{15} \mathrm{H}_{19} \mathrm{NO}_{3} \mathrm{~F}_{3} \mathrm{~S}[\mathrm{M}+\mathrm{H}]^{+}$350.1032, found: 350.1036 .

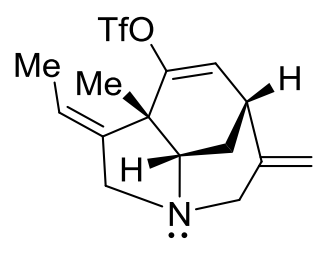

19

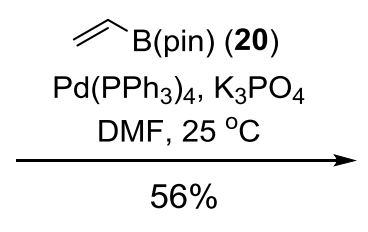

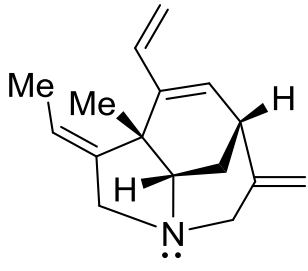

21

Diene 21: To a stirred solution of $19(120 \mathrm{mg}, 0.34 \mathrm{mmol})$ in DMF (3.5 mL) was added pinacol vinylboronate 20 (105 mg, $0.68 \mathrm{mmol}), \mathrm{Pd}\left(\mathrm{PPh}_{3}\right)_{4}(39 \mathrm{mg}, 0.034 \mathrm{mmol})$ and $\mathrm{K}_{3} \mathrm{PO}_{4}(216 \mathrm{mg}, 1.02$ $\mathrm{mmol})$. The resulting mixture was stirred at $25^{\circ} \mathrm{C}$ for $16 \mathrm{~h}$ before it was quenched with brine $(20 \mathrm{~mL})$ and extracted with $t$-BuOMe $(3 \times 10 \mathrm{~mL})$. The combined organic phases were dried over anhydrous $\mathrm{Na}_{2} \mathrm{SO}_{4}$, filtered, and concentrated under vacuum. The residue so obtained was purified by flash column chromatography with EtOAc/MeOH (50:1 $\rightarrow$ 10:1) to give diene 21 (43 mg, 0.19 mmol, 56\%) as a light yellow oil. Data for 21: $R_{\mathrm{f}}=0.50$ (ethyl acetate:methanol 10:1); $[\mathrm{a}]_{\mathrm{D}}^{12}=+17.9(c=1.0$, $\left.\mathrm{CHCl}_{3}\right) ;{ }^{1} \mathrm{H} \mathrm{NMR}\left(400 \mathrm{MHz}, \mathrm{CDCl}_{3}\right): \delta=6.49-6.39(\mathrm{~m}, 1 \mathrm{H}), 5.77(\mathrm{~d}, J=6.4 \mathrm{~Hz}, 1 \mathrm{H}), 5.55-5.47(\mathrm{~m}$, $1 \mathrm{H}), 5.27(\mathrm{dd}, J=17.0,2.0 \mathrm{~Hz}, 1 \mathrm{H}), 5.02-4.91(\mathrm{~m}, 1 \mathrm{H}), 4.78(\mathrm{~s}, 1 \mathrm{H}), 4.76(\mathrm{~s}, 1 \mathrm{H}), 3.50(\mathrm{~d}, J=$ $13.9 \mathrm{~Hz}, 1 \mathrm{H}), 3.40$ (d, $J=13.5 \mathrm{~Hz}, 1 \mathrm{H}), 3.20$ (d, $J=14.1 \mathrm{~Hz}, 1 \mathrm{H}), 3.13-2.99$ (m, $2 \mathrm{H}), 2.96-2.89$ $(\mathrm{m}, 1 \mathrm{H}), 1.98-1.84(\mathrm{~m}, 2 \mathrm{H}), 1.83(\mathrm{~d}, J=7.1 \mathrm{~Hz}, 3 \mathrm{H}), 1.33(\mathrm{~s}, 3 \mathrm{H}) \mathrm{ppm} ;{ }^{13} \mathrm{C} \mathrm{NMR}(101 \mathrm{MHz}$, $\left.\mathrm{CDCl}_{3}\right): \delta=146.5,144.9,143.1,137.6,125.8,118.6,114.4,108.2,65.7,61.8,53.0,47.5,36.8,25.5$, 23.2, $15.2 \mathrm{ppm}$; IR (thin film): $v_{\max }=2932,1777,1727,1654,1444,1379,1251,1164,1068,993$, 909, $801 \mathrm{~cm}^{-1}$; HRMS (ESI): Calcd for $\mathrm{C}_{16} \mathrm{H}_{22} \mathrm{~N}[\mathrm{M}+\mathrm{H}]^{+} 228.1747$, found: 228.1751 .

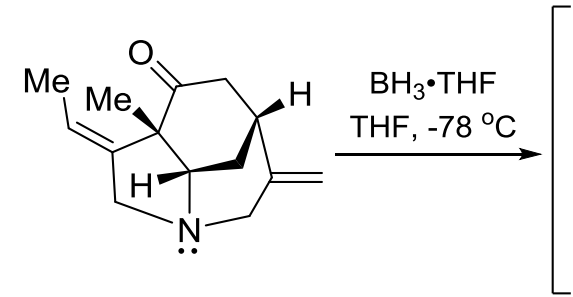

13

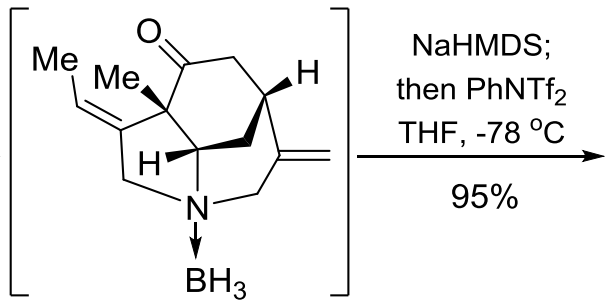

23

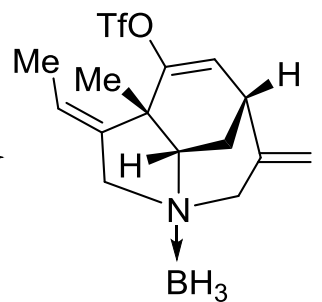

24

$N$-borane complexed ketone 23 and triflate 24: To a stirred solution of $\mathbf{1 3}$ (1.62 g, $7.45 \mathrm{mmol})$ in THF (70 mL) was slowly added $\mathrm{BH}_{3} \bullet \mathrm{THF}(7.5 \mathrm{~mL}, 1.0 \mathrm{M}$ in THF, $7.5 \mathrm{mmol})$ at $-78^{\circ} \mathrm{C}$. After stirred at that temperature for $30 \mathrm{~min}$, NaHMDS (4.5 mL, 2.0 M in THF, $9.0 \mathrm{mmol}$ ) was added. The resulting 
mixture was stirred at $-78{ }^{\circ} \mathrm{C}$ for $40 \mathrm{~min}$ before $\operatorname{PhNTf}_{2}(3.20 \mathrm{~g}, 9.0 \mathrm{mmol})$ in $\mathrm{THF}(15 \mathrm{~mL})$ was slowly added. The resulting mixture was gradually warmed to $0{ }^{\circ} \mathrm{C}$ in $6 \mathrm{~h}$ before it was quenched with brine $(60 \mathrm{~mL})$ and extracted with EtOAc $(3 \times 60 \mathrm{~mL})$. The combined organic phases were dried over anhydrous $\mathrm{Na}_{2} \mathrm{SO}_{4}$, filtered, and concentrated under vacuum. The residue so obtained was purified by flash column chromatography with EtOAc/petroleum ether/ $\mathrm{CH}_{2} \mathrm{Cl}_{2}(1: 40: 1 \rightarrow 1: 10: 1)$ to give $N$ borane complexed triflate $24(2.57 \mathrm{~g}, 7.08 \mathrm{mmol}, 95 \%)$ as a white crystalline solid. Data for $\mathbf{2 4}: R_{\mathrm{f}}=$ 0.40 (EtOAc:petroleum ether 1:16); m.p. $=92-94{ }^{\circ} \mathrm{C} ;[\mathrm{a}]_{\mathrm{D}}^{30}=+16.0\left(c=1.0, \mathrm{CHCl}_{3}\right) ;{ }^{1} \mathrm{H}$ NMR $(400$ $\left.\mathrm{MHz}, \mathrm{CDCl}_{3}\right): \delta=5.81(\mathrm{~d}, J=7.3 \mathrm{~Hz}, 1 \mathrm{H}), 5.79-5.72(\mathrm{~m}, 1 \mathrm{H}), 5.09(\mathrm{~s}, 1 \mathrm{H}), 5.02(\mathrm{~s}, 1 \mathrm{H}), 3.98$ $3.88(\mathrm{~m}, 1 \mathrm{H}), 3.54(\mathrm{~d}, J=15.2 \mathrm{~Hz}, 1 \mathrm{H}), 3.50-3.41(\mathrm{~m}, 3 \mathrm{H}), 3.22-3.16(\mathrm{~m}, 1 \mathrm{H}), 2.51$ (dt, $J=13.5$, $2.8 \mathrm{~Hz}, 1 \mathrm{H}), 1.92-1.87$ (m, $3 \mathrm{H}), 1.86-1.80$ (m, $1 \mathrm{H}), 1.55$ (s, $3 \mathrm{H}) \mathrm{ppm} ;{ }^{13} \mathrm{C} \mathrm{NMR}\left(101 \mathrm{MHz}, \mathrm{CDCl}_{3}\right)$ : $\delta=151.8,138.1,135.1,125.7,118.7,118.4(\mathrm{q}, J=319.8 \mathrm{~Hz}), 114.5,74.0,70.1,57.6,47.6,36.0,22.8$, 22.5, $15.1 \mathrm{ppm} ;{ }^{11} \mathrm{~B}$ NMR (128 MHz, $\left.\mathrm{CDCl}_{3}\right): \delta=-11.95 \mathrm{ppm}$; IR (thin film): $v_{\max }=2933,2369,2324$, 2278, 1444, 1394, 1219, 1173, 1131, 999, 912, 889, 847, 599, $511 \mathrm{~cm}^{-1}$; HRMS (ESI): Calcd for $\mathrm{C}_{15} \mathrm{H}_{19} \mathrm{NO}_{3} \mathrm{~F}_{3} \mathrm{~S}$ [M-BH $\left.3+\mathrm{H}\right]^{+} 350.1032$, found: 350.1035; CCDC 1893591 contains the supplementary crystallographic data for $\mathbf{2 4}$ and is available free of charge from The Cambridge Crystallographic Data Centre via www.ccdc.cam.ac.uk/data_request/cif.

In the above procedure, by removing the volatiles under vacuum before NaHMDS was added, intermediate $\mathbf{2 3}$ could be obtained in quantitative yield as a white crystalline solid. Data for 23: $R_{\mathrm{f}}=$ 0.40 (EtOAc:petroleum ether 1:12); m.p. $=129-130{ }^{\circ} \mathrm{C} ;[\mathrm{a}]_{\mathrm{D}}^{12}=-37.4\left(c=1.0, \mathrm{CHCl}_{3}\right) ;{ }^{1} \mathrm{H} \mathrm{NMR}(400$ $\left.\mathrm{MHz}, \mathrm{CDCl}_{3}\right): \delta=5.74(\mathrm{q}, J=7.2 \mathrm{~Hz}, 1 \mathrm{H}), 5.04(\mathrm{~s}, 1 \mathrm{H}), 4.94(\mathrm{~s}, 1 \mathrm{H}), 3.95-3.79(\mathrm{~m}, 1 \mathrm{H}), 3.54$ 3.40 (m, 2 H), 3.34-3.29 (m, 1 H), 3.11 (d, $J=14.5$ Hz, 1 H), 2.99-2.92 (m, 1 H), 2.77-2.68 (m, 2 H), 2.50-2.40 (m, $1 \mathrm{H}), 2.06-2.01(\mathrm{~m}, 1 \mathrm{H}), 2.00-1.95(\mathrm{~m}, 3 \mathrm{H}), 1.54$ (s, $3 \mathrm{H}) \mathrm{ppm} ;{ }^{13} \mathrm{C}$ NMR (101 MHz, $\left.\mathrm{CDCl}_{3}\right): \delta=210.4,141.2,134.8,125.4,114.3,73.4,69.8,56.9,45.9,36.7,23.4,22.3,15.3 \mathrm{ppm} ;{ }^{11} \mathrm{~B}$ NMR (128 MHz, $\mathrm{CDCl}_{3}$ ): $\delta=-12.39 \mathrm{ppm}$; IR (thin film): $v_{\max }=2934,2369,2324,1705,1448,1174$, 1107, 1026, 974, 910, 832, $666 \mathrm{~cm}^{-1}$; HRMS (ESI): Calcd for $\mathrm{C}_{14} \mathrm{H}_{22} \mathrm{NOBNa}[\mathrm{M}+\mathrm{Na}]^{+} 254.1687$, found: 254.1690; CCDC 1893590 contains the supplementary crystallographic data for $\mathbf{2 3}$ and is available free of charge from The Cambridge Crystallographic Data Centre via www.ccdc.cam.ac.uk/data_request/cif.

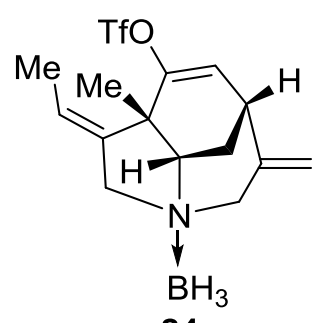

24

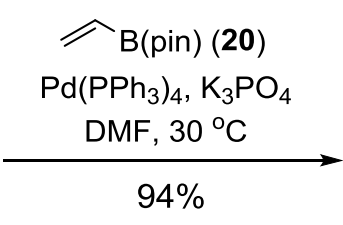

$94 \%$

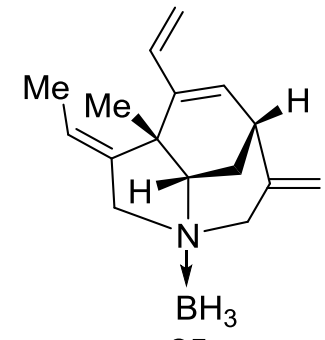

25 
Diene 25: To a stirred solution of $24(380 \mathrm{mg}, 1.05 \mathrm{mmol})$ in DMF (10 mL) was added pinacol vinylboronate 20 (322 mg, $2.10 \mathrm{mmol}), \mathrm{Pd}\left(\mathrm{PPh}_{3}\right)_{4}(121 \mathrm{mg}, 0.105 \mathrm{mmol})$ and $\mathrm{K}_{3} \mathrm{PO}_{4}(667 \mathrm{mg}, 3.14$ $\mathrm{mmol})$. The resulting mixture was stirred at $30^{\circ} \mathrm{C}$ for $36 \mathrm{~h}$ before it was quenched with water $(60 \mathrm{~mL})$ and extracted with $t$-BuOMe $(3 \times 60 \mathrm{~mL})$. The combined organic phases were dried over anhydrous $\mathrm{Na}_{2} \mathrm{SO}_{4}$, filtered, and concentrated under vacuum. The residue so obtained was purified by flash column chromatography with EtOAc/petroleum ether $(1: 40 \rightarrow 1: 20)$ to give diene 25 (237 $\mathrm{mg}, 0.98$ mmol, 94\%) as a white crystalline solid. Data for 25: $R_{\mathrm{f}}=0.60$ (ethyl acetate: petroleum ether 1:16); $[\mathrm{a}]_{\mathrm{D}}^{26}=+32.8\left(c=1.0, \mathrm{CHCl}_{3}\right) ;{ }^{1} \mathrm{H} \mathrm{NMR}\left(400 \mathrm{MHz}, \mathrm{CDCl}_{3}\right): \delta=6.42(\mathrm{dd}, J=17.0,10.8 \mathrm{~Hz}, 1 \mathrm{H})$, $5.80(\mathrm{~d}, J=6.9 \mathrm{~Hz}, 1 \mathrm{H}), 5.57(\mathrm{qt}, J=7.4,1.9 \mathrm{~Hz}, 1 \mathrm{H}), 5.29(\mathrm{dd}, J=17.0,2.0 \mathrm{~Hz}, 1 \mathrm{H}), 5.04(\mathrm{dd}, J$ = 10.6, $2.0 \mathrm{~Hz}, 1 \mathrm{H}), 4.99(\mathrm{~s}, 1 \mathrm{H}), 4.91(\mathrm{~s}, 1 \mathrm{H}), 3.79-3.72(\mathrm{~m}, 1 \mathrm{H}), 3.53-3.37$ (m, 3 H), 3.41-3.32 (m, $1 \mathrm{H}), 3.02-2.96(\mathrm{~m}, 1 \mathrm{H}), 2.37(\mathrm{dt}, J=13.6,2.5 \mathrm{~Hz}, 1 \mathrm{H}), 1.86(\mathrm{dt}, J=7.4,1.8 \mathrm{~Hz}, 3 \mathrm{H}), 1.79$ $1.74(\mathrm{~m}, 1 \mathrm{H}), 1.42$ (s, $3 \mathrm{H}) \mathrm{ppm} ;{ }^{13} \mathrm{C} \mathrm{NMR}\left(101 \mathrm{MHz}, \mathrm{CDCl}_{3}\right): \delta=142.5,141.0,138.4,137.0,126.1$, 121.8, 115.8, 112.6, 72.7, 69.5, 58.6, 47.7, 35.2, 24.0, 22.2, 15.7 ppm; ${ }^{11} \mathrm{~B}$ NMR (128 MHz, $\left.\mathrm{CDCl}_{3}\right)$ : $\delta=-11.43$ ppm; IR (thin film): $v_{\max }=2962,2936,2369,2323,2273,1440,1172,1091,1059,998$, 908, $830 \mathrm{~cm}^{-1}$; HRMS (ESI): Calcd for $\mathrm{C}_{16} \mathrm{H}_{24} \mathrm{BNNa}[\mathrm{M}+\mathrm{Na}]^{+}$264.1894, found: 264.1896; CCDC 1899997 contains the supplementary crystallographic data for $\mathbf{2 5}$ and is available free of charge from The Cambridge Crystallographic Data Centre via www.ccdc.cam.ac.uk/data_request/cif.

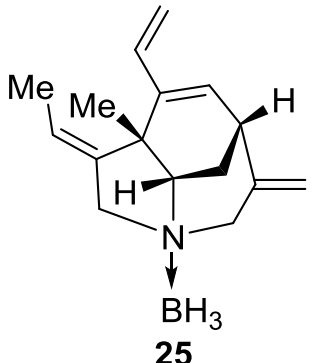

25

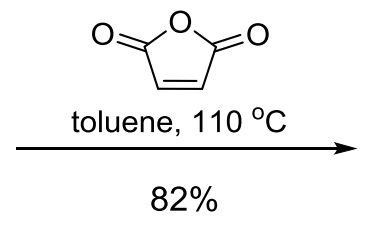

$82 \%$

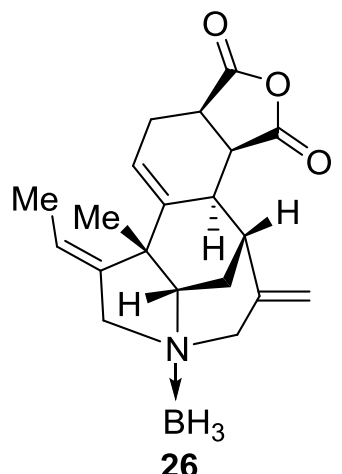

26

Cycloadduct 26: To a stirred solution of 25 (48 $\mathrm{mg}, 0.20 \mathrm{mmol})$ in toluene $(5 \mathrm{~mL})$ was added maleic anhydride $(78 \mathrm{mg}, 0.80 \mathrm{mmol})$. After stirring at $110{ }^{\circ} \mathrm{C}$ for $2 \mathrm{~h}$, the reaction mixture was directly subjected to flash column chromatography using EtOAc/petroleum ether $(1: 10 \rightarrow 1: 5)$ as eluent to give the cycloadduct $26(56 \mathrm{mg}, 0.16 \mathrm{mmol}, 82 \%)$ as a white crystalline solid. Data for $\mathbf{2 6}: R_{\mathrm{f}}=0.40$ (EtOAc:petroleum ether 1:4); m.p. $=164-167{ }^{\circ} \mathrm{C} ;[\mathrm{a}]_{\mathrm{D}}^{30}=-68.3\left(c=1.0, \mathrm{CHCl}_{3}\right) ;{ }^{1} \mathrm{H} \mathrm{NMR}(400 \mathrm{MHz}$, $\left.\mathrm{CDCl}_{3}\right): \delta=6.41-6.29(\mathrm{~m}, 1 \mathrm{H}), 5.55-5.41(\mathrm{~m}, 1 \mathrm{H}), 5.04(\mathrm{~s}, 1 \mathrm{H}), 4.90(\mathrm{~s}, 1 \mathrm{H}), 3.98-3.86(\mathrm{~m}, 1 \mathrm{H})$, 3.53-3.47 (m, $1 \mathrm{H}), 3.46-3.37(\mathrm{~m}, 3 \mathrm{H}), 3.30(\mathrm{~d}, J=13.3 \mathrm{~Hz}, 1 \mathrm{H}), 3.18-3.11(\mathrm{~m}, 1 \mathrm{H}), 3.08-3.02$ (s, $1 \mathrm{H}), 2.94-2.84(\mathrm{~m}, 1 \mathrm{H}), 2.48(\mathrm{dt}, J=14.9,3.1 \mathrm{~Hz}, 1 \mathrm{H}), 2.29-2.22(\mathrm{~m}, 1 \mathrm{H}), 2.18-2.03(\mathrm{~m}, 2 \mathrm{H})$, $1.94(\mathrm{~d}, J=7.5 \mathrm{~Hz}, 3 \mathrm{H}), 1.43(\mathrm{~s}, 3 \mathrm{H}) \mathrm{ppm} ;{ }^{13} \mathrm{C} \mathrm{NMR}\left(101 \mathrm{MHz}, \mathrm{CDCl}_{3}\right): \delta=173.5,172.4,146.1$, 
144.2, 141.6, 126.4, 120.2, 112.3, 72.3, 70.2, 58.0, 47.0, 46.9, 41.1, 41.0, 35.9, 29.4, 25.0, 20.4, 15.5 ppm; ${ }^{11} \mathrm{~B}$ NMR (128 MHz, $\left.\mathrm{CDCl}_{3}\right): \delta=-11.97 \mathrm{ppm}$; IR (thin film): $v_{\max }=2926,2856,2364,1849$, 1775, 1447, 1244, 1176, 1109, 1073, 969, 939, 904, $736 \mathrm{~cm}^{-1}$; HRMS (ESI): Calcd for $\mathrm{C}_{20} \mathrm{H}_{25} \mathrm{BNO}_{3}[\mathrm{M}-\mathrm{H}]^{-}$338.1927, found: 338.1930; CCDC 1893592 contains the supplementary crystallographic data for $\mathbf{2 6}$ and is available free of charge from The Cambridge Crystallographic Data Centre via www.ccdc.cam.ac.uk/data_request/cif.

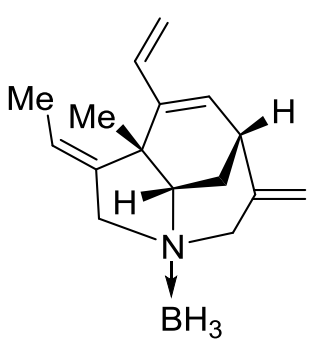

25

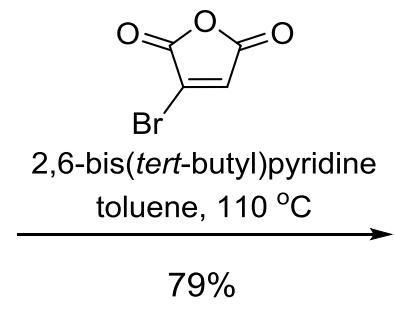

Cycloadduct 27: To a stirred solution of 25 (45 mg, $0.19 \mathrm{mmol})$ in toluene $(5 \mathrm{~mL})$ was added bromomaleic anhydride (132 mg, $0.75 \mathrm{mmol})$ and 2, 6-bis(tert-butyl)pyridine (285 $\mathrm{mg}, 1.49 \mathrm{mmol})$. After stirring at $110{ }^{\circ} \mathrm{C}$ for $2 \mathrm{~h}$, the reaction mixture was directly subjected to flash column chromatography using EtOAc/petroleum ether $(1: 10 \rightarrow 1: 4)$ as eluent to give the cycloadduct 27 (61 $\mathrm{mg}, 0.15 \mathrm{mmol}, 79 \%$ ) as a white crystalline solid. Data for 27: $R_{\mathrm{f}}=0.30$ (EtOAc:petroleum ether 1:4); m.p. $=177-180{ }^{\circ} \mathrm{C} ;[\mathrm{a}]_{\mathrm{D}}^{14}=+71.5\left(c=1.0, \mathrm{CHCl}_{3}\right) ;{ }^{1} \mathrm{H} \mathrm{NMR}\left(400 \mathrm{MHz}, \mathrm{CDCl}_{3}\right): \delta=6.10-5.96(\mathrm{~m}, 1$ H), 5.59-5.49 (m, $1 \mathrm{H}), 5.46(\mathrm{~s}, 1 \mathrm{H}), 5.22(\mathrm{~s}, 1 \mathrm{H}), 4.42-4.23(\mathrm{~m}, 1 \mathrm{H}), 4.12(\mathrm{~d}, J=14.9 \mathrm{~Hz}, 1 \mathrm{H})$, 3.95-3.85 (m, $1 \mathrm{H}), 3.65(\mathrm{~d}, J=14.9 \mathrm{~Hz}, 1 \mathrm{H}), 3.55-3.39(\mathrm{~m}, 2 \mathrm{H}), 3.34-3.17$ (m, $2 \mathrm{H}), 2.87-2.81(\mathrm{~m}$, $1 \mathrm{H}), 2.05-1.95(\mathrm{~m}, 1 \mathrm{H}), 1.62-1.59(\mathrm{~m}, 1 \mathrm{H}), 1.57$ (d, J=7.2 Hz, $3 \mathrm{H}), 1.49$ (s, $3 \mathrm{H}) \mathrm{ppm} ;{ }^{13} \mathrm{C}$ NMR $\left(101 \mathrm{MHz}, \mathrm{CDCl}_{3}\right): \delta=164.0,163.7,144.8,142.2,141.4,138.5,137.4,122.2,119.2,116.2,74.3$, 67.5, 59.1, 49.4, 38.5, 37.6, 24.8, 23.5, 23.4, 12.4 ppm; ${ }^{11} \mathrm{~B}$ NMR (128 MHz, $\left.\mathrm{CDCl}_{3}\right): \delta=-4.25 \mathrm{ppm}$; IR (thin film): $v_{\max }=2968,2431,1847,1772,1720,1618,1266,1179,1102,897,815,722 \mathrm{~cm}^{-1}$; HRMS (ESI): Calcd for $\mathrm{C}_{20} \mathrm{H}_{22} \mathrm{NO}_{3}\left[\mathrm{M}-\mathrm{BH}_{2} \mathrm{Br}+\mathrm{H}\right]^{+}$324.1594, found: 324.1598; CCDC 1893593 contains the supplementary crystallographic data for $\mathbf{2 7}$ and is available free of charge from The Cambridge Crystallographic Data Centre via www.ccdc.cam.ac.uk/data_request/cif.

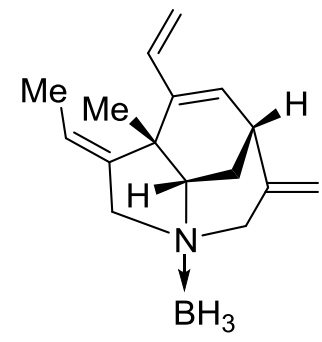

25

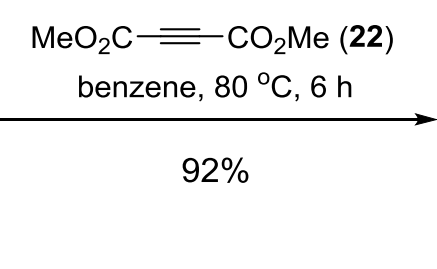

$92 \%$

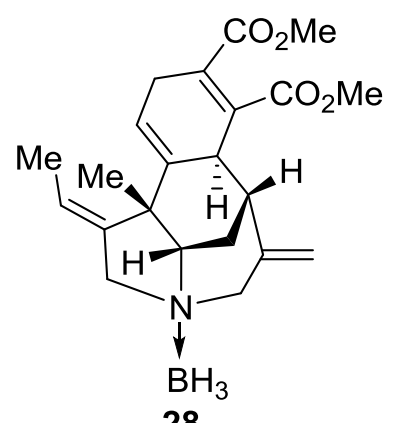

28 
Cycloadduct 28: To a stirred solution of 25 (250 mg, $1.04 \mathrm{mmol})$ in benzene (10 mL) was added dimethyl acetylenedicarboxylate (22) $(295 \mathrm{mg}, 2.07 \mathrm{mmol})$. After stirring at $80{ }^{\circ} \mathrm{C}$ for $6 \mathrm{~h}$, the reaction mixture was directly subjected to flash column chromatography using EtOAc/petroleum ether (1:10 $\rightarrow 1: 5)$ as eluent to give the cycloadduct $\mathbf{2 8}$ (366 $\mathrm{mg}, 0.95 \mathrm{mmol}, 92 \%)$ as a colorless oil. Data for $\mathbf{2 8}$ : $R_{\mathrm{f}}=0.40$ (EtOAc:petroleum ether $\left.1: 4\right) ;[\mathrm{a}]_{\mathrm{D}}^{12}=+79.8\left(c=1.0, \mathrm{CHCl}_{3}\right) ;{ }^{1} \mathrm{H} \mathrm{NMR}\left(400 \mathrm{MHz}, \mathrm{CDCl}_{3}\right)$ : $\delta=5.89-5.84(\mathrm{~m}, 1 \mathrm{H}), 5.67-5.49(\mathrm{~m}, 1 \mathrm{H}), 4.85(\mathrm{~s}, 1 \mathrm{H}), 4.80(\mathrm{~s}, 1 \mathrm{H}), 3.88(\mathrm{~d}, J=13.9 \mathrm{~Hz}, 1 \mathrm{H})$, 3.75 (s, 3 H), 3.74 (s, 3 H), 3.70-3.60 (m, 2 H), 3.44-3.38 (s, 1 H), 3.28 (d, J = 14.0 Hz, 1 H), $3.23-$ $3.11(\mathrm{~m}, 2 \mathrm{H}), 3.06-2.94(\mathrm{~m}, 1 \mathrm{H}), 2.65-2.60(\mathrm{~m}, 1 \mathrm{H}), 2.03-1.96(\mathrm{~m}, 1 \mathrm{H}), 1.60(\mathrm{~d}, J=5.1 \mathrm{~Hz}, 3 \mathrm{H})$, 1.58-1.54 (m, $1 \mathrm{H}), 1.38$ (s, $3 \mathrm{H}) \mathrm{ppm} ;{ }^{13} \mathrm{C} \mathrm{NMR}\left(101 \mathrm{MHz}, \mathrm{CDCl}_{3}\right): \delta=169.0,166.8,143.5,139.8$, $139.4,135.3,126.2,122.9,120.0,111.5,76.4,70.5,58.5,52.4,52.2,49.0,39.8,38.8,27.5,24.7,23.9$, $12.3 \mathrm{ppm} ;{ }^{11} \mathrm{~B}$ NMR (128 MHz, $\mathrm{CDCl}_{3}$ ): $\delta=-10.43 \mathrm{ppm}$; IR (thin film): $v_{\max }=2952,2370,2321$, 1725, 1652, 1435, 1270, 1232, 1171, 1088, 1061, 961, $904 \mathrm{~cm}^{-1}$; HRMS (ESI): Calcd for $\mathrm{C}_{22} \mathrm{H}_{30} \mathrm{NO}_{4} \mathrm{BNa}[\mathrm{M}+\mathrm{Na}]^{+}$406.2160, found: 406.2165.

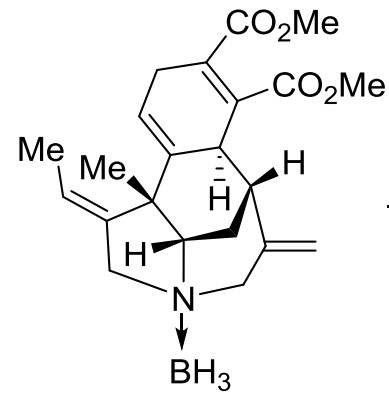

28

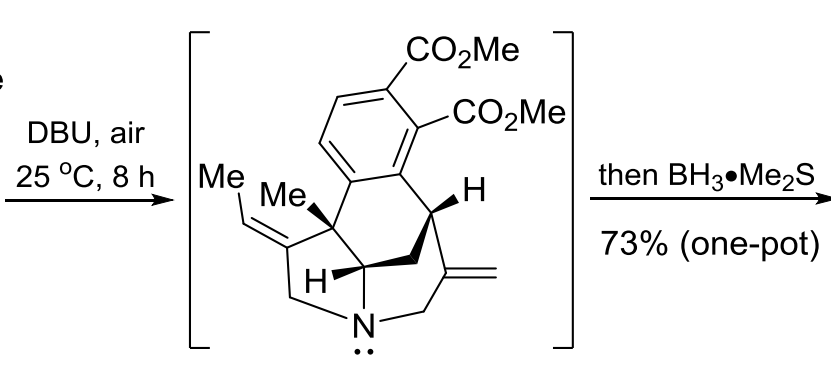

$29(69 \%$ isolated yield $)$

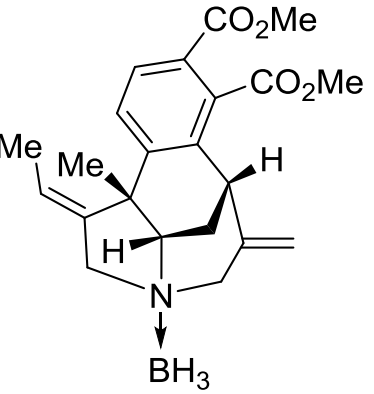

30

Tetracyclic core 29 and 30: A mixture of cyclohexadiene 28 (52 mg, $0.136 \mathrm{mmol})$ and 1,8diazabicyclo[5.4.0]undec-7-ene (DBU) $(310 \mathrm{mg}, 2.04 \mathrm{mmol})$ was stirred under open air at room temperature for $8 \mathrm{~h}$. The reaction system was then charged with argon and diluted with $\mathrm{CH}_{2} \mathrm{Cl}_{2}(10$ $\mathrm{mL}), \mathrm{BH}_{3} \cdot \mathrm{Me}_{2} \mathrm{~S}(0.41 \mathrm{~mL}, 10 \mathrm{M}, 4.1 \mathrm{mmol})$ was slowly added at $-78{ }^{\circ} \mathrm{C}$. After stirred at that temperature for $30 \mathrm{~min}$, the reaction mixture was quenched with $\mathrm{MeOH}(2 \mathrm{~mL})$ and concentrated under vacuum. The residue so obtained was purified by flash column chromatography with EtOAc/petroleum ether $(1: 10 \rightarrow 1: 6)$ to give $N$-borane complexed tetracyclic core 30 (38 mg, 0.010 mmol, 73\%) as a white crystalline solid. Data for 30: $R_{\mathrm{f}}=0.50$ (EtOAc:petroleum ether 1:6); m.p. $=$ $131-132{ }^{\circ} \mathrm{C} ;[\mathrm{a}]_{\mathrm{D}}^{10}=-57.2\left(c=1.0, \mathrm{CHCl}_{3}\right) ;{ }^{1} \mathrm{H} \mathrm{NMR}\left(400 \mathrm{MHz}, \mathrm{CDCl}_{3}\right): \delta=7.90(\mathrm{~d}, J=8.4 \mathrm{~Hz}, 1$ H), $7.80(\mathrm{~d}, J=8.4 \mathrm{~Hz}, 1 \mathrm{H}), 5.67$ (q, $J=7.3 \mathrm{~Hz}, 1 \mathrm{H}), 5.07(\mathrm{~s}, 1 \mathrm{H}), 4.93(\mathrm{~s}, 1 \mathrm{H}), 4.04-3.96(\mathrm{~m}, 1$ H), 3.92 (s, $3 \mathrm{H}), 3.91-3.84(\mathrm{~m}, 1 \mathrm{H}), 3.87$ (s, $3 \mathrm{H}), 3.53$ (d, J=15.3 Hz, $1 \mathrm{H}), 3.44-3.33$ (m, 2 H), $3.07(\mathrm{~d}, J=13.2 \mathrm{~Hz}, 1 \mathrm{H}), 2.70(\mathrm{dt}, J=14.0,3.3 \mathrm{~Hz}, 1 \mathrm{H}), 2.15(\mathrm{~d}, J=7.5 \mathrm{~Hz}, 3 \mathrm{H}), 2.07-2.01(\mathrm{~m}, 1$ $\mathrm{H}), 1.66$ (s, $3 \mathrm{H}) \mathrm{ppm} ;{ }^{13} \mathrm{C} \mathrm{NMR}\left(101 \mathrm{MHz}, \mathrm{CDCl}_{3}\right): \delta=169.0,165.8,147.4,140.6,140.2,134.8$, $134.8,129.7,128.9,126.8,121.7,113.9,72.4,70.3,58.0,52.7,52.6,48.5,36.9,29.1,23.0,15.8$ ppm; 
${ }^{11} \mathrm{~B}$ NMR (128 MHz, $\left.\mathrm{CDCl}_{3}\right): \delta=-12.63 \mathrm{ppm}$; IR (thin film): $v_{\max }=2951,2364,2328,1728,1436$, 1275, 1261, 1153, 912, $750 \mathrm{~cm}^{-1}$; HRMS (ESI): Calcd for $\mathrm{C}_{22} \mathrm{H}_{28} \mathrm{NO}_{4} \mathrm{BNa}[\mathrm{M}+\mathrm{Na}]^{+} 404.2004$, found: 404.2004; CCDC 1899998 contains the supplementary crystallographic data for $\mathbf{3 0}$ and is available free of charge from The Cambridge Crystallographic Data Centre via www.ccdc.cam.ac.uk/data_request/cif.

In the above procedure, by repeated flash column chromatography with EtOAc/MeOH $(10: 1 \rightarrow 5: 1)$ before $\mathrm{BH}_{3} \cdot \mathrm{Me}_{2} \mathrm{~S}$ was added, tetracyclic core 29 could also be separated in $69 \%$ yield as a colorless oil. Data for 29: $R_{\mathrm{f}}=0.40$ (EtOAc: methanol 4:1); $[\mathrm{a}]_{\mathrm{D}}^{10}=-45.8\left(c=1.0, \mathrm{CHCl}_{3}\right) ;{ }^{1} \mathrm{H} \mathrm{NMR}(400 \mathrm{MHz}$, $\left.\mathrm{CDCl}_{3}\right): \delta=7.85(\mathrm{~d}, J=8.4 \mathrm{~Hz}, 1 \mathrm{H}), 7.77(\mathrm{~d}, J=8.4 \mathrm{~Hz}, 1 \mathrm{H}), 5.58(\mathrm{q}, J=7.4 \mathrm{~Hz}, 1 \mathrm{H}), 4.86(\mathrm{~s}, 1$ H), 4.80 (s, 1 H), 3.90 (s, 3 H), 3.86 (s, 3 H), 3.84-3.80 (m, 1 H), 3.76-3.68 (m, 1 H), 3.26-3.17 (m, $2 \mathrm{H}), 3.16(\mathrm{t}, J=2.8 \mathrm{~Hz}, 1 \mathrm{H}), 2.67(\mathrm{~d}, J=12.6 \mathrm{~Hz}, 1 \mathrm{H}), 2.25-2.19(\mathrm{~m}, 2 \mathrm{H}), 2.13$ (d, $J=7.4 \mathrm{~Hz}, 3$ $\mathrm{H}), 1.58$ (s, $3 \mathrm{H}) \mathrm{ppm} ;{ }^{13} \mathrm{C} \mathrm{NMR}\left(101 \mathrm{MHz}, \mathrm{CDCl}_{3}\right): \delta=169.3,166.0,150.2,147.9,145.7,135.1$, 134.8, 129.8, 128.3 , 125.8, 118.8, 108.6, 65.9, 62.5, 53.6, 52.4, 52.3, 48.5, 38.5, 28.3, 27.0, 15.5 ppm; IR (thin film): $v_{\max }=2948,1727,1434,1293,1271,1240,1195,1151,977,894,738 \mathrm{~cm}^{-1} ; \mathrm{HRMS}$ (ESI): Calcd for $\mathrm{C}_{22} \mathrm{H}_{26} \mathrm{NO}_{4}[\mathrm{M}+\mathrm{H}]^{+}$368.1856, found: 368.1860 . 


\section{II $\quad{ }^{1} \mathrm{H}$ and ${ }^{13} \mathrm{C}$ NMR Spectra of Compounds}

${ }^{1} \mathrm{H}$ NMR Spectra Comparison of $\mathbf{1 4}$ and $\mathbf{1 4 a}\left(400 \mathrm{MHz}, \mathrm{CDCl}_{3}\right)$

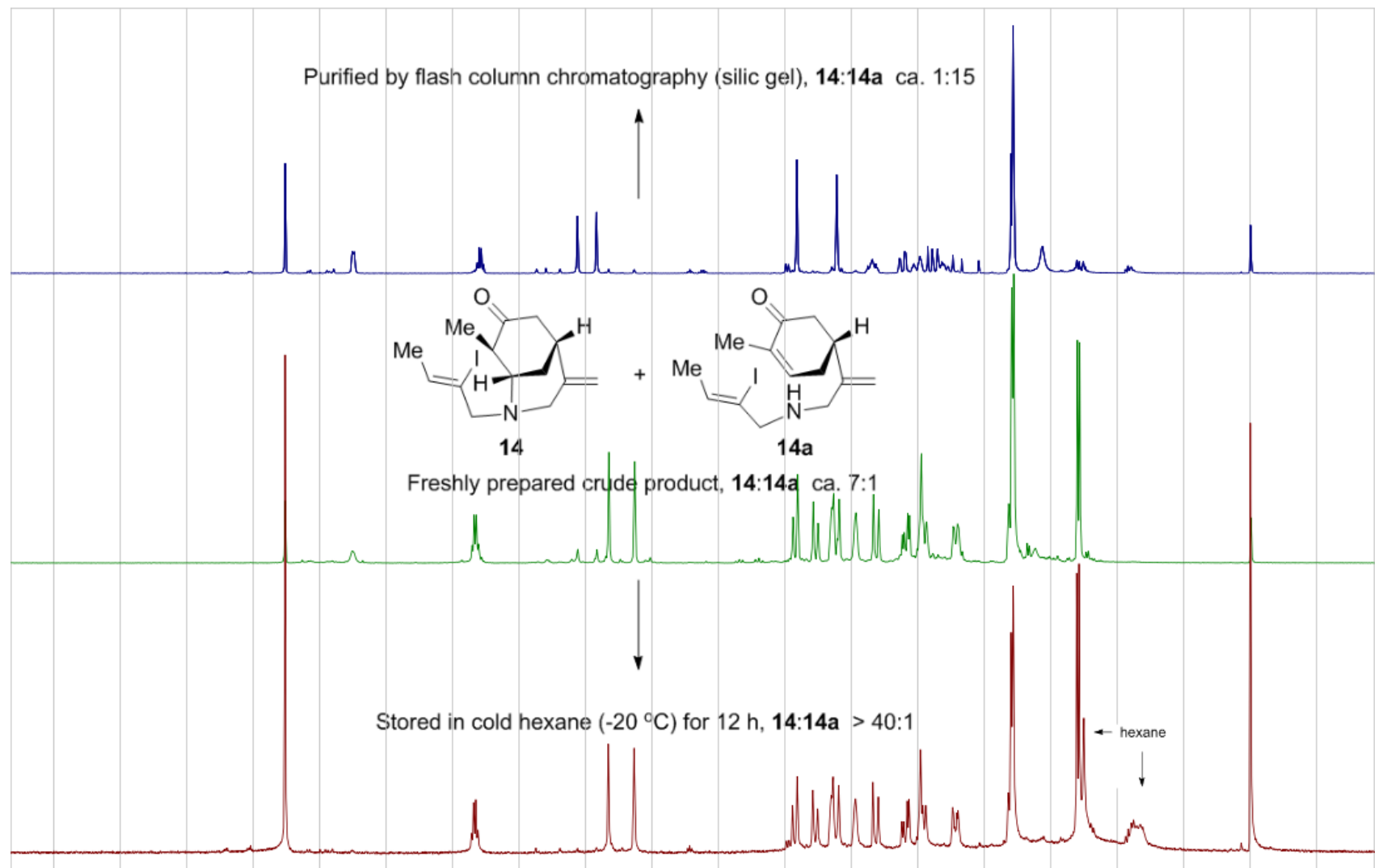

$\begin{array}{llllllllllllllllllllll}9.0 & 0.5 & 0.0 & 7.5 & 7.0 & 6.5 & 6.0 & 5.5 & 5.0 & 4.5 & 4.0 & 3.5 & 3.0 & 2.5 & 2.0 & 1.5 & 1.0 & 0.5 & 0.0 & -0.5\end{array}$

${ }^{13} \mathrm{C}$ NMR Spectra of $\mathbf{1 4 a}\left(101 \mathrm{MHz}, \mathrm{CDCl}_{3}\right)$

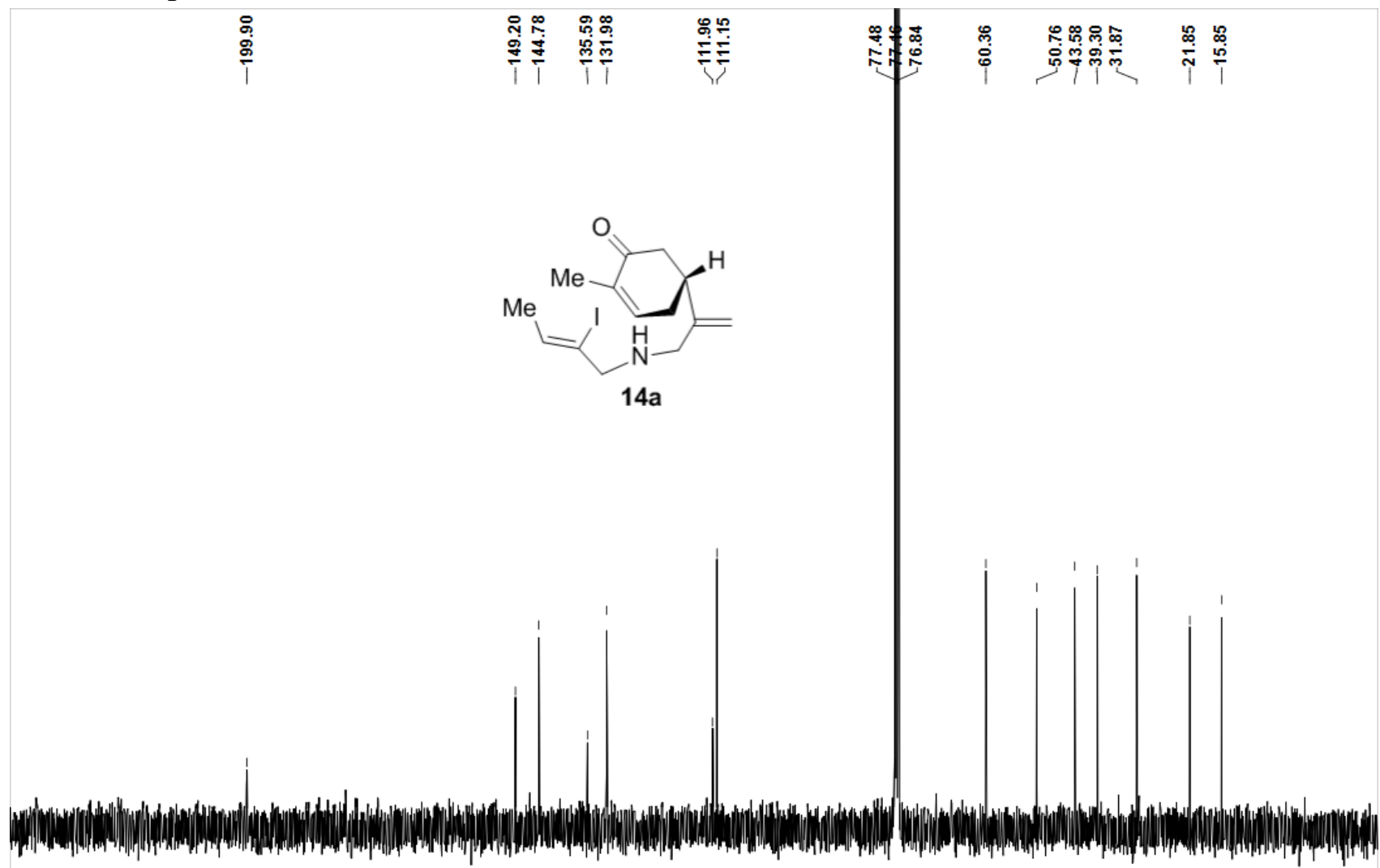


${ }^{1} \mathrm{H}$ NMR Spectra of $14\left(400 \mathrm{MHz}, \mathrm{CDCl}_{3}\right)$

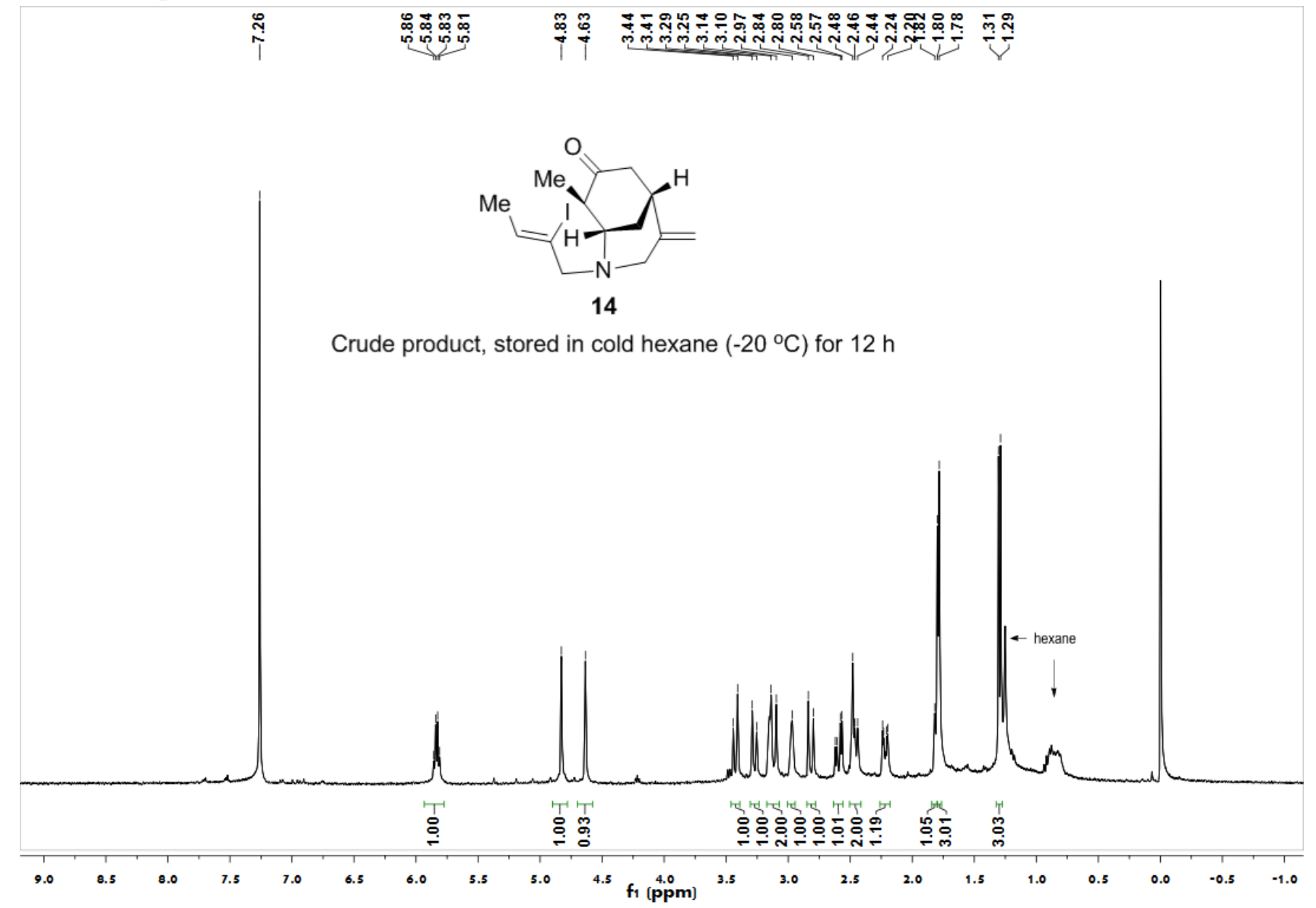

${ }^{13} \mathrm{C}$ NMR Spectra of $14\left(101 \mathrm{MHz}, \mathrm{CDCl}_{3}\right)$

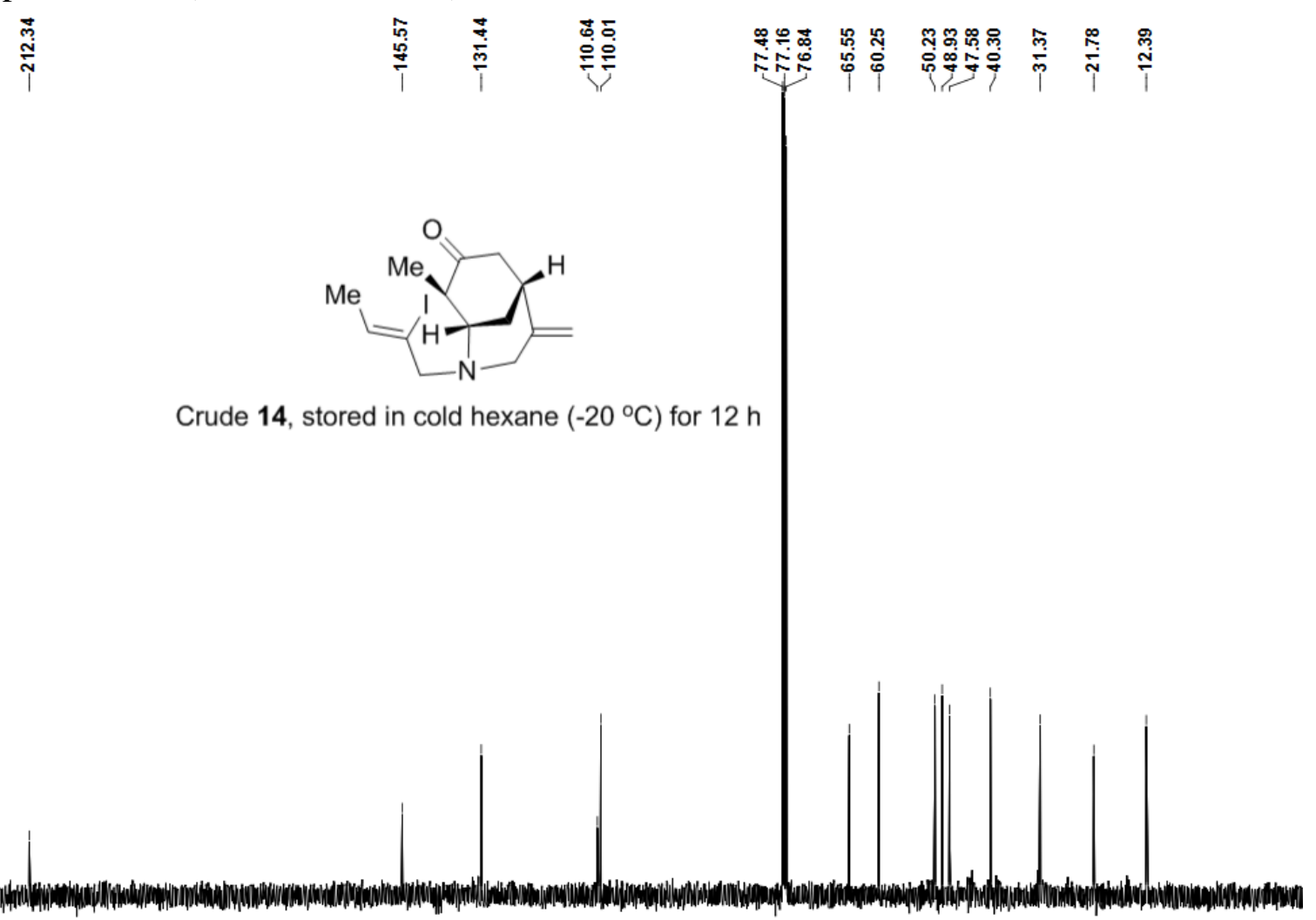

$\begin{array}{llllllllllllllllllllllllllll}240 & 230 & 220 & 210 & 200 & 190 & 180 & 170 & 160 & 150 & 140 & 130 & 120 & 110 & 100 & 90 & 80 & 70 & 60 & 50 & 40 & 30 & 20 & 10 & 0 & -10\end{array}$ 
${ }^{1} \mathrm{H}_{-}{ }^{13} \mathrm{C}$ HSQC of $14\left(400 \mathrm{MHz}, \mathrm{CDCl}_{3}\right)$

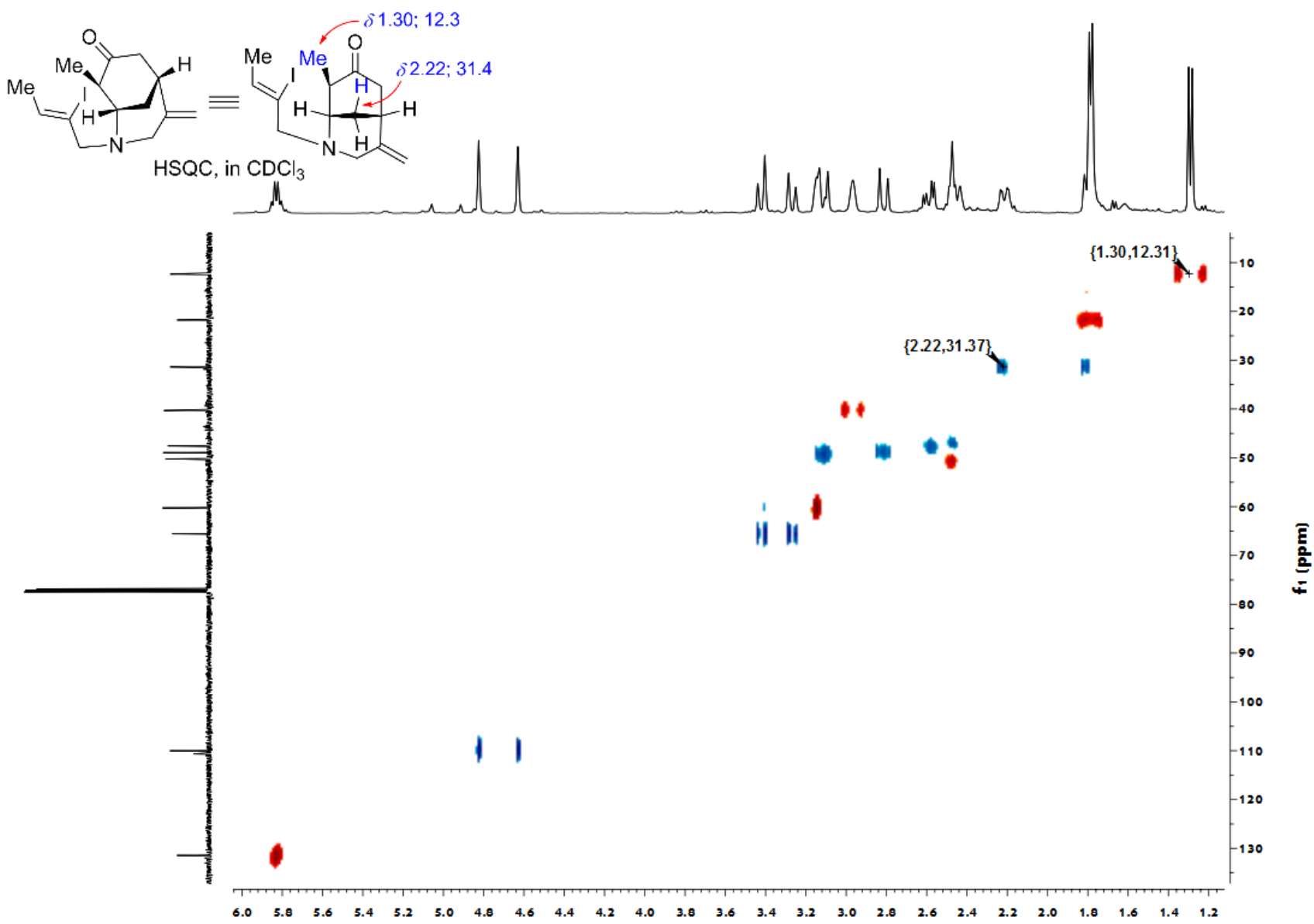

${ }^{1} \mathrm{H}-{ }^{1} \mathrm{H}$ NOESY of $14\left(400 \mathrm{MHz}, \mathrm{CDCl}_{3}\right)$

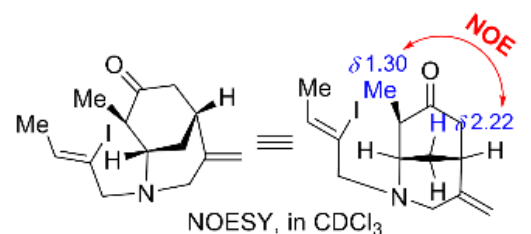

NOESY, in $\mathrm{CDCl}_{3}$

$\mathrm{M}^{3}$

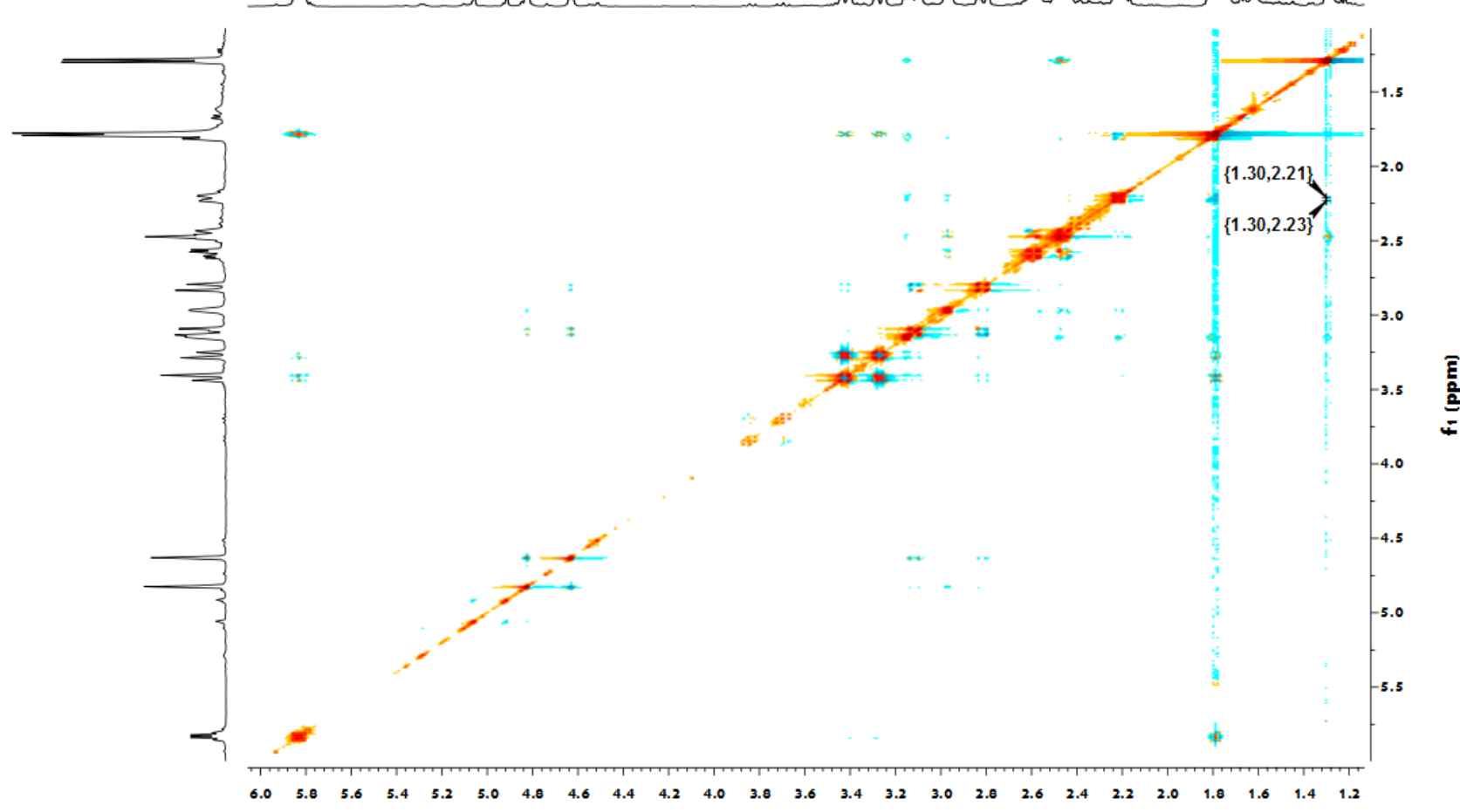


${ }^{1} \mathrm{H}$ NMR Spectra of $13\left(400 \mathrm{MHz}, \mathrm{CDCl}_{3}\right)$

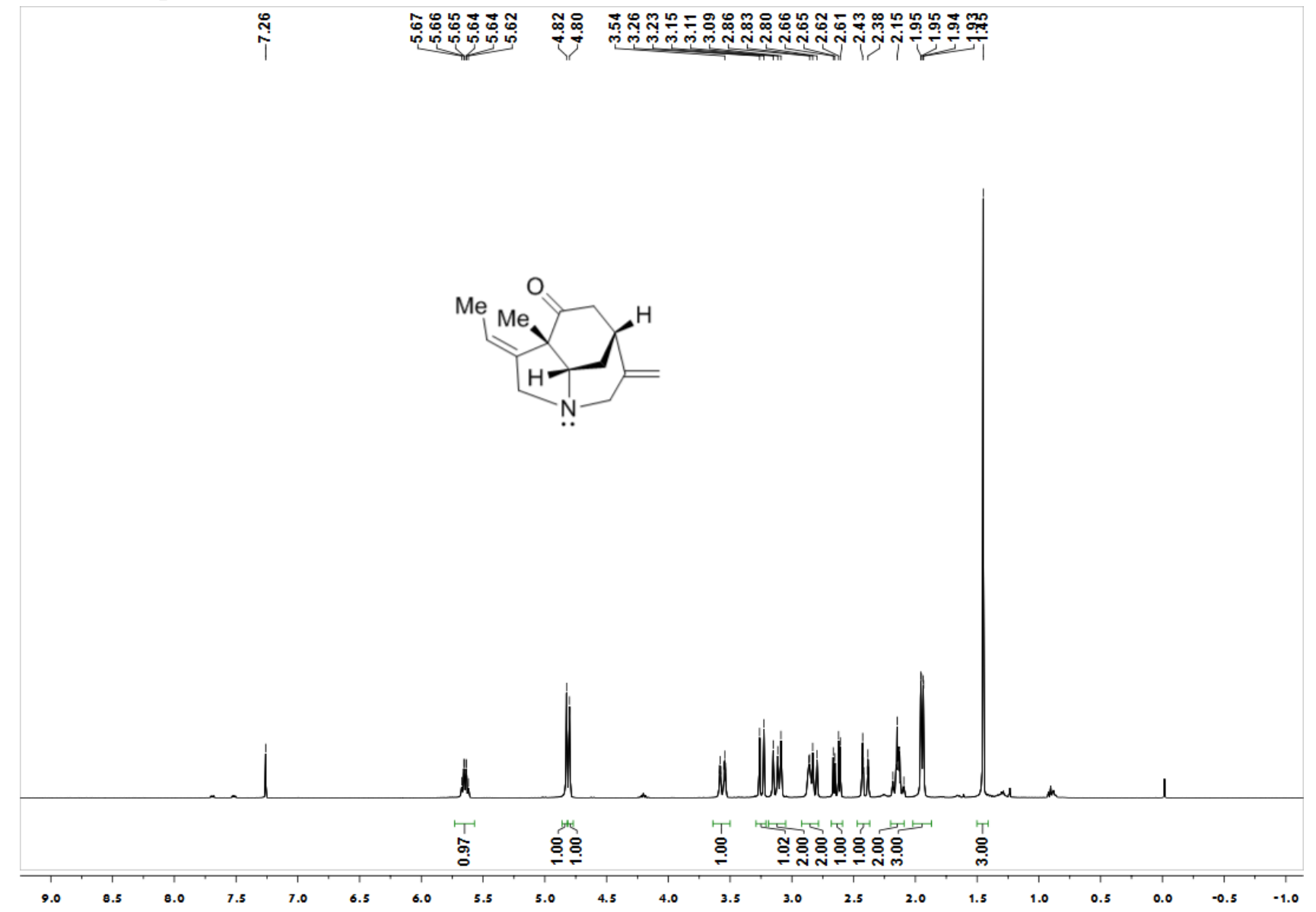

${ }^{13} \mathrm{C}$ NMR Spectra of $13\left(101 \mathrm{MHz}, \mathrm{CDCl}_{3}\right)$

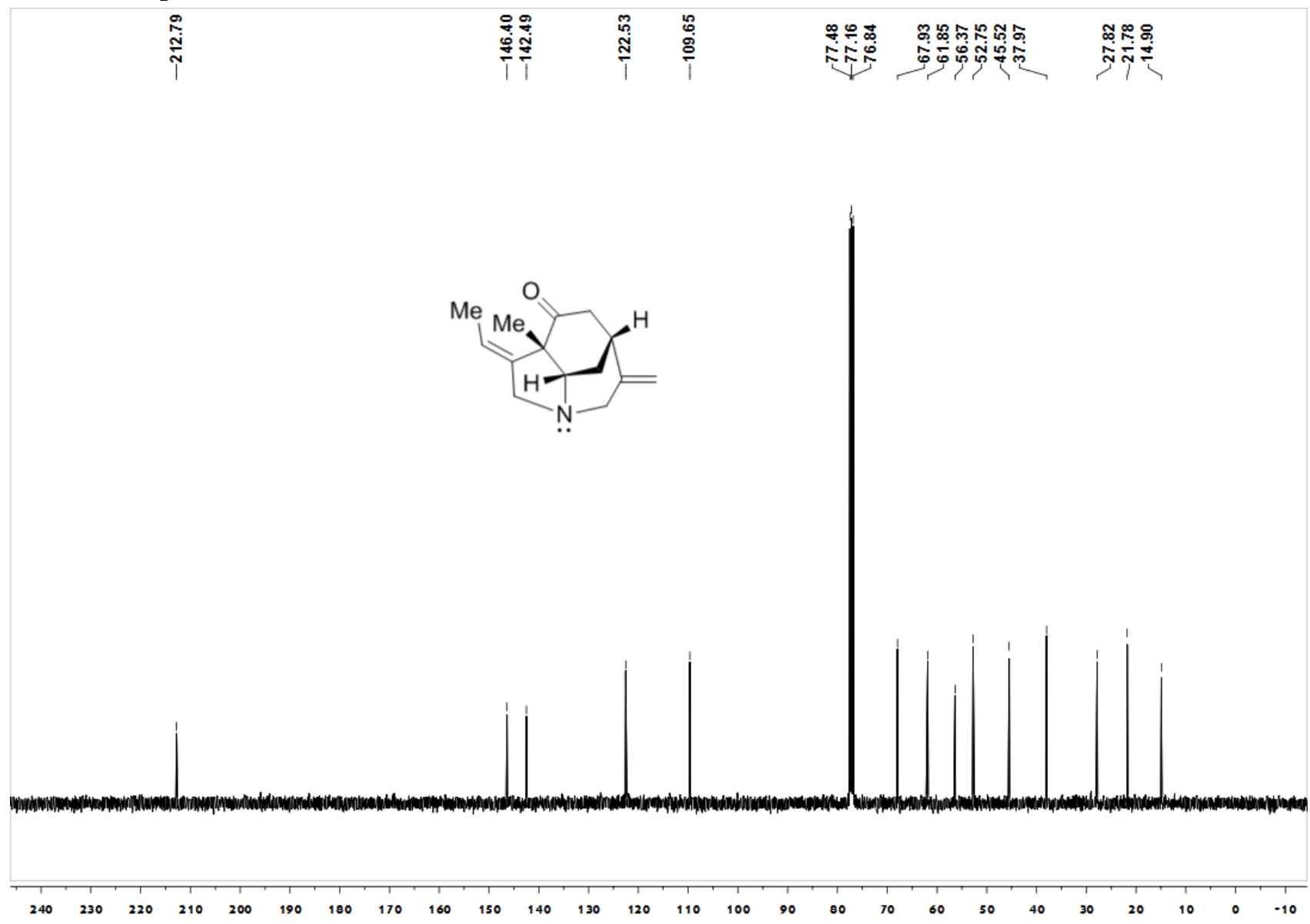


${ }^{1} \mathrm{H}$ NMR Spectra of $17\left(400 \mathrm{MHz}, \mathrm{CDCl}_{3}\right)$

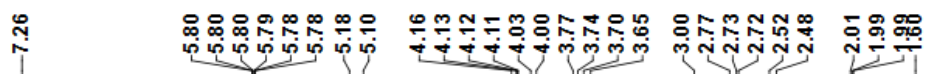
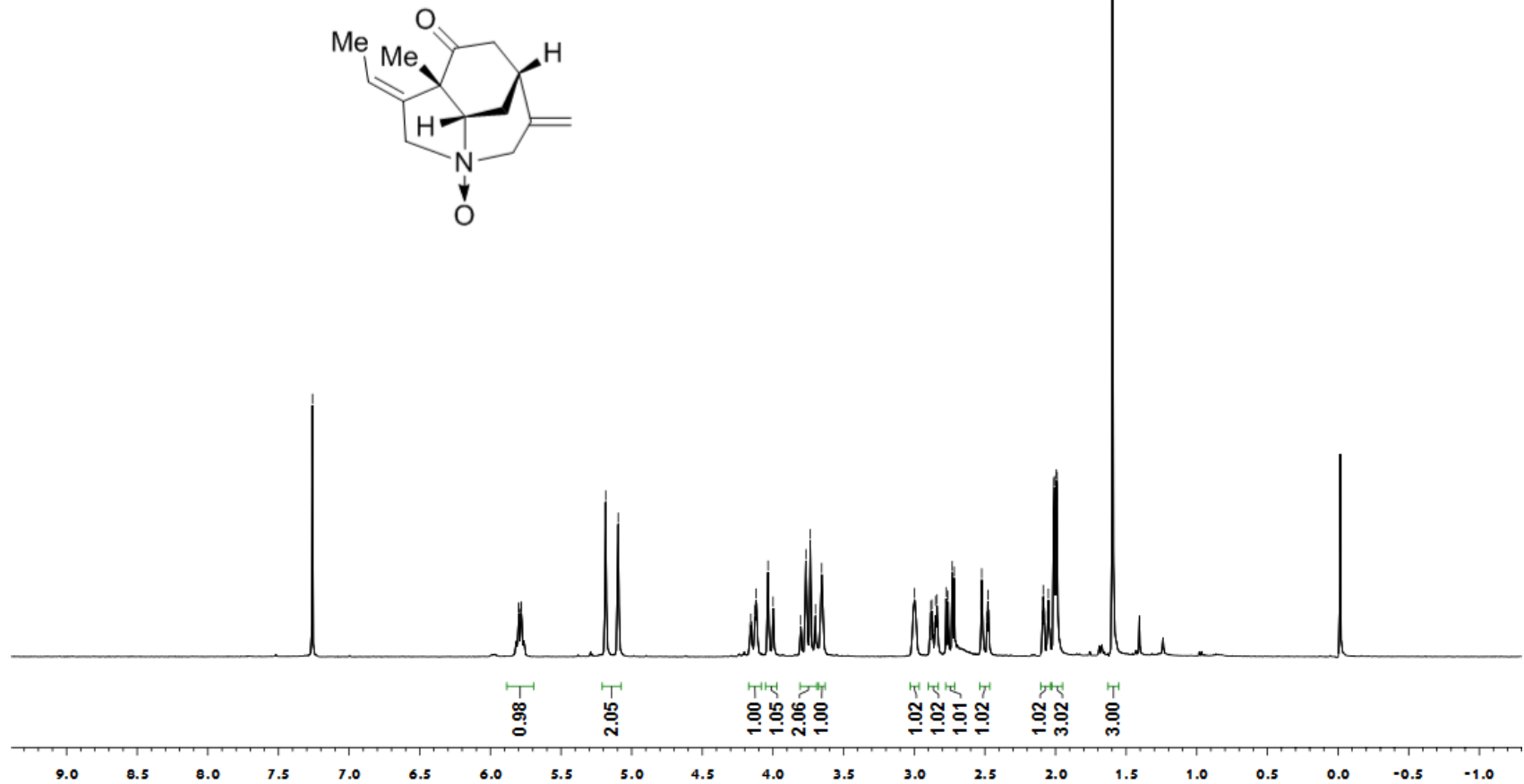

${ }^{13} \mathrm{C}$ NMR Spectra of $17\left(101 \mathrm{MHz}, \mathrm{CDCl}_{3}\right)$

恶1<smiles>C=C1CN(O)CC2(C)CC(=O)CC1CC2=O</smiles>

$\begin{array}{llllllllllllllllllllllllll}240 & 230 & 220 & 210 & 200 & 190 & 180 & 170 & 160 & 150 & 140 & 130 & 120 & 110 & 100 & 90 & 80 & 70 & 60 & 50 & 40 & 30 & 20 & 10 & 0 & -10\end{array}$ 
${ }^{1} \mathrm{H}$ NMR Spectra of $19\left(400 \mathrm{MHz}, \mathrm{CDCl}_{3}\right)$
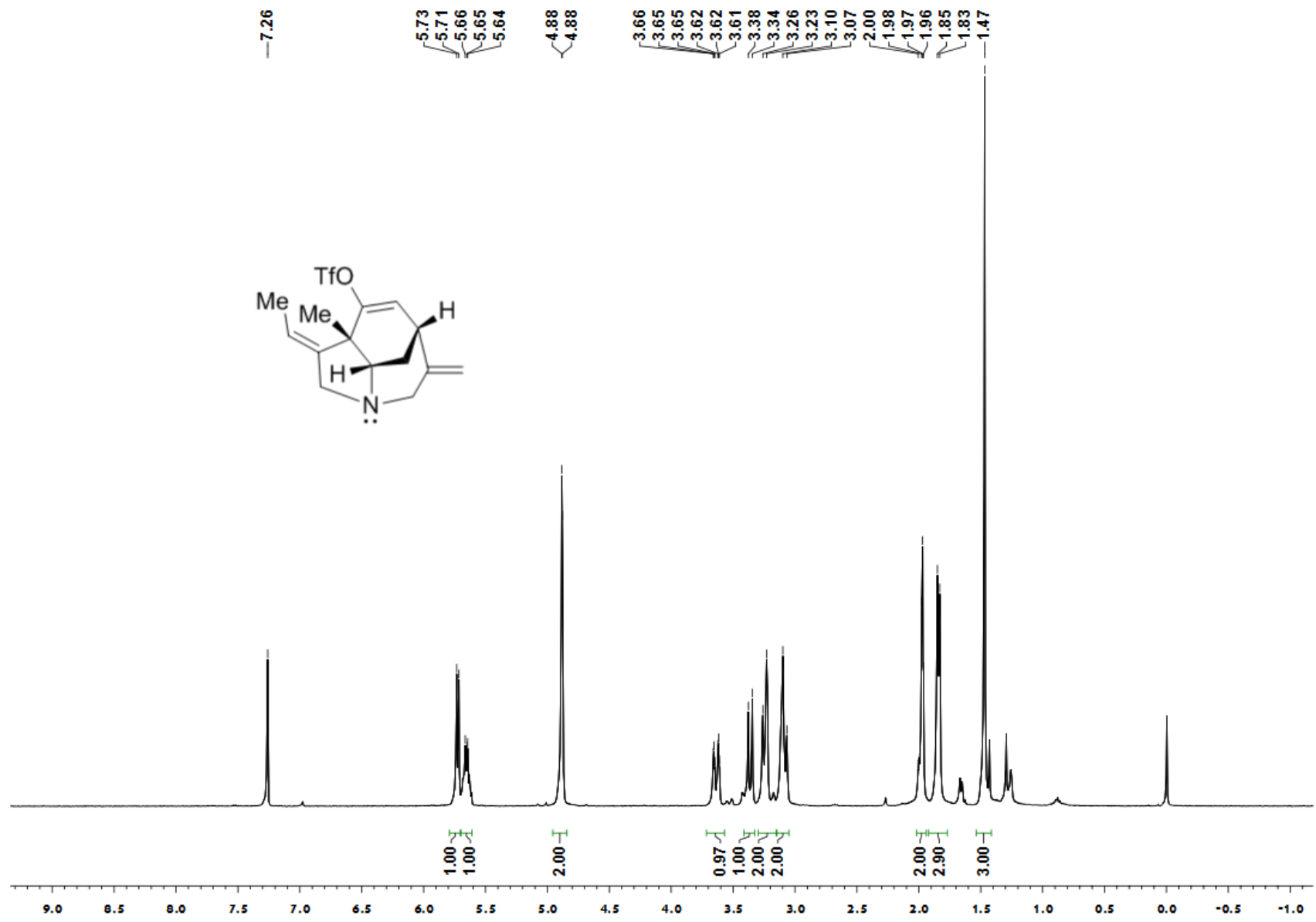

${ }^{13} \mathrm{C}$ NMR Spectra of 19 (101 MHz, $\left.\mathrm{CDCl}_{3}\right)$
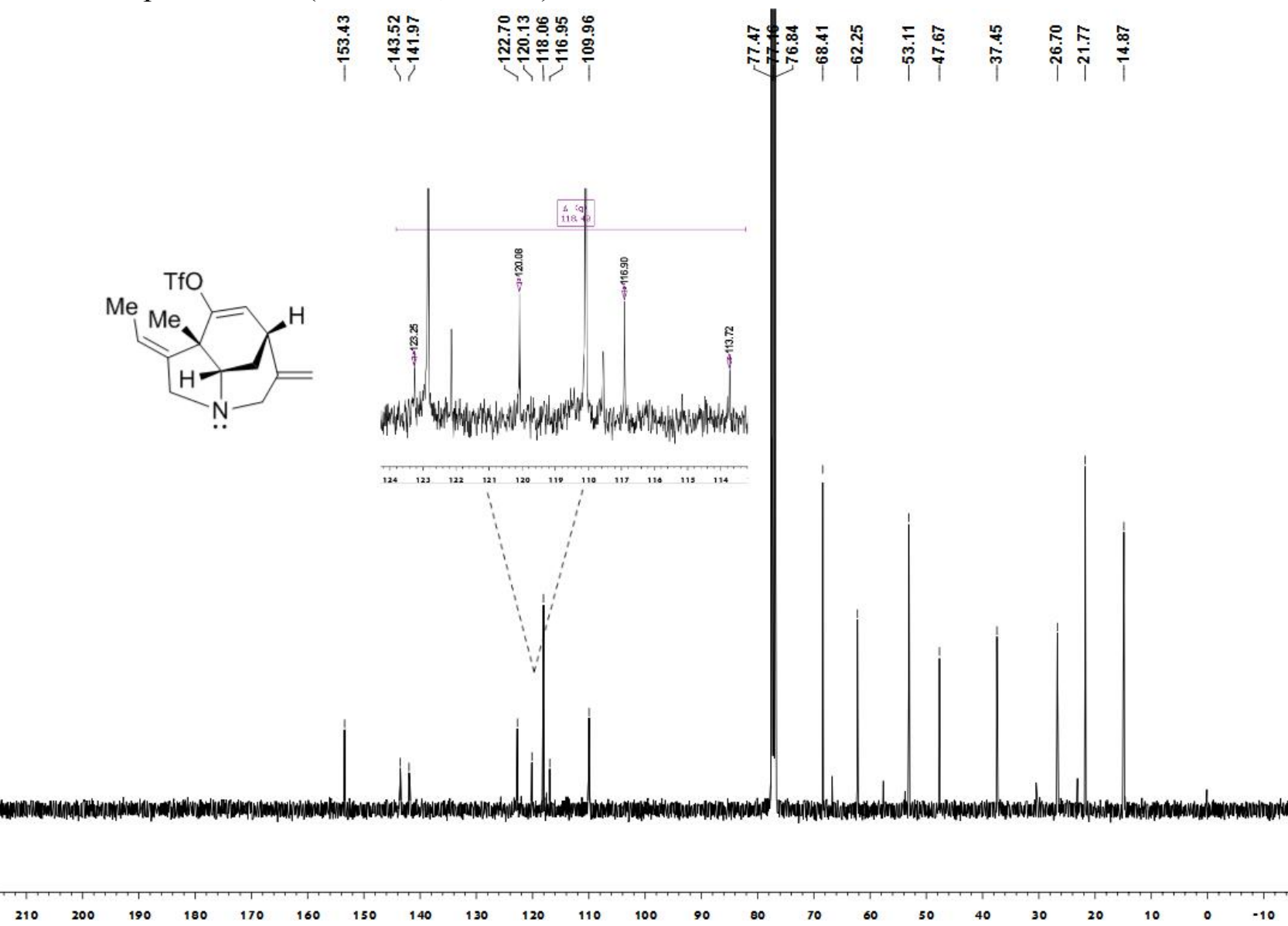
${ }^{1} \mathrm{H}$ NMR Spectra of $21\left(400 \mathrm{MHz}, \mathrm{CDCl}_{3}\right)$

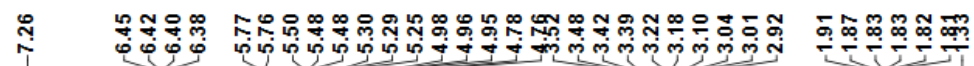
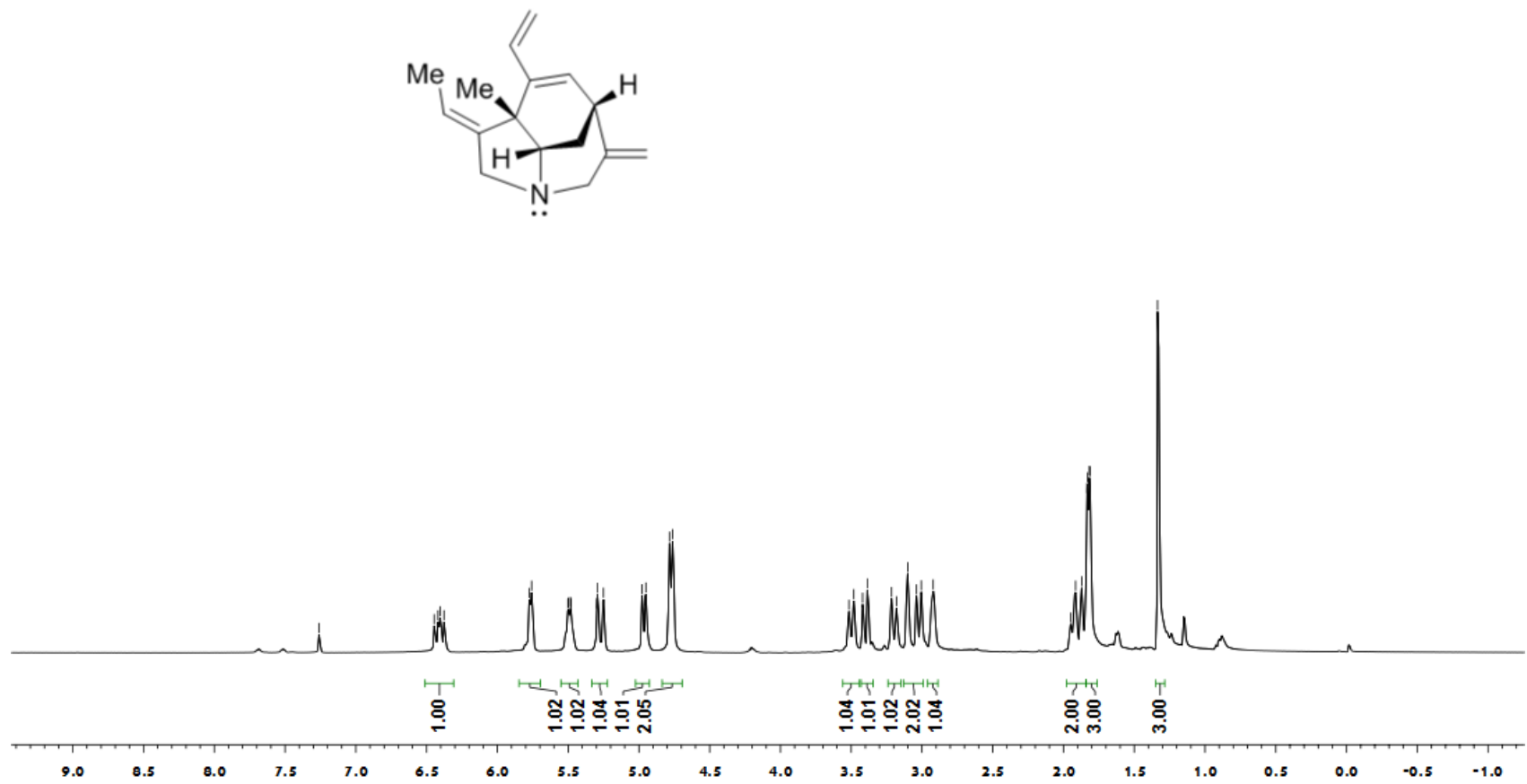

${ }^{13} \mathrm{C}$ NMR Spectra of $21\left(101 \mathrm{MHz}, \mathrm{CDCl}_{3}\right)$

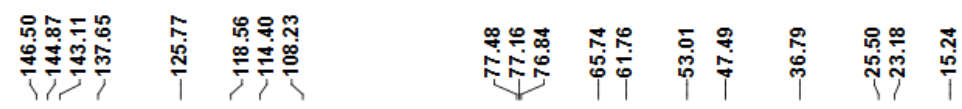
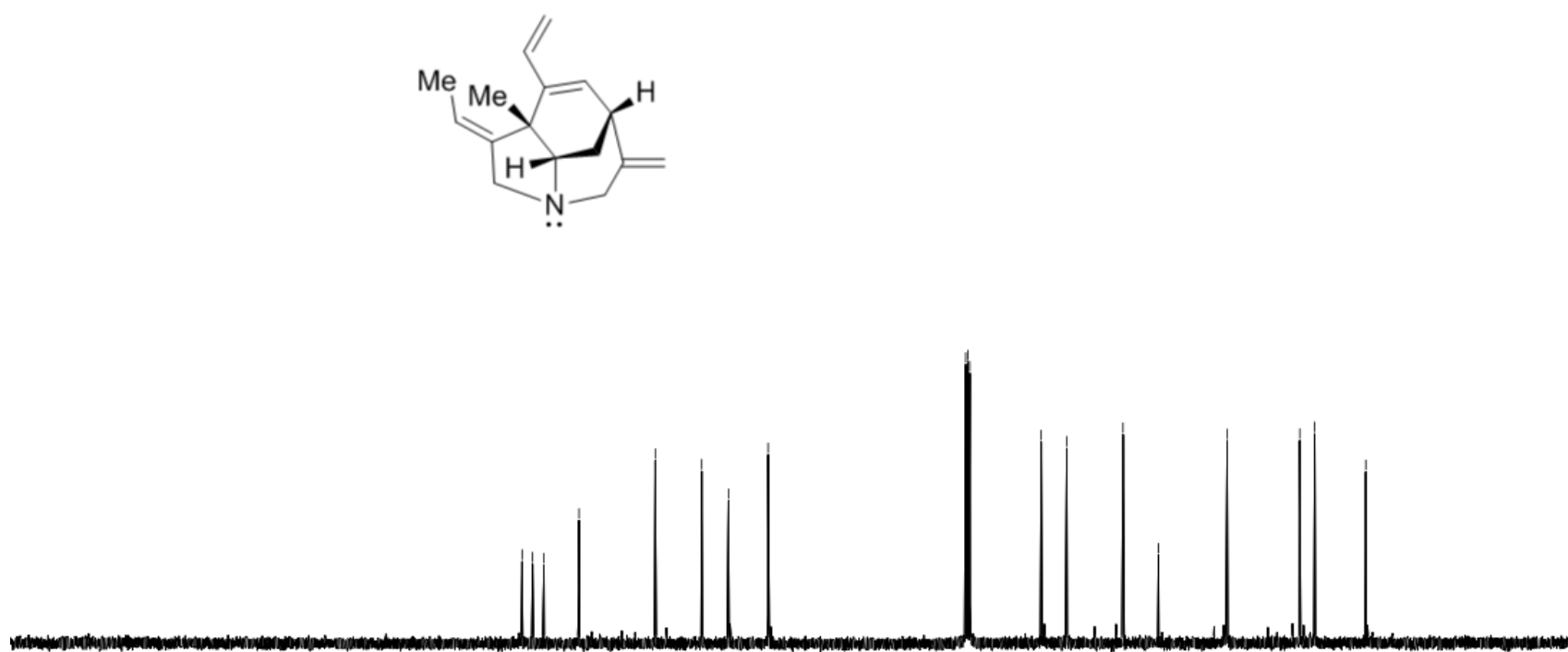

$\begin{array}{llllllllllllllllllllllllll}220 & 210 & 200 & 190 & 180 & 170 & 160 & 150 & 140 & 130 & 120 & 110 & 100 & 90 & 80 & 70 & 60 & 50 & 40 & 30 & 20 & 10 & 0 & -10\end{array}$ 
${ }^{1} \mathrm{H}$ NMR Spectra of $23\left(400 \mathrm{MHz}, \mathrm{CDCl}_{3}\right)$
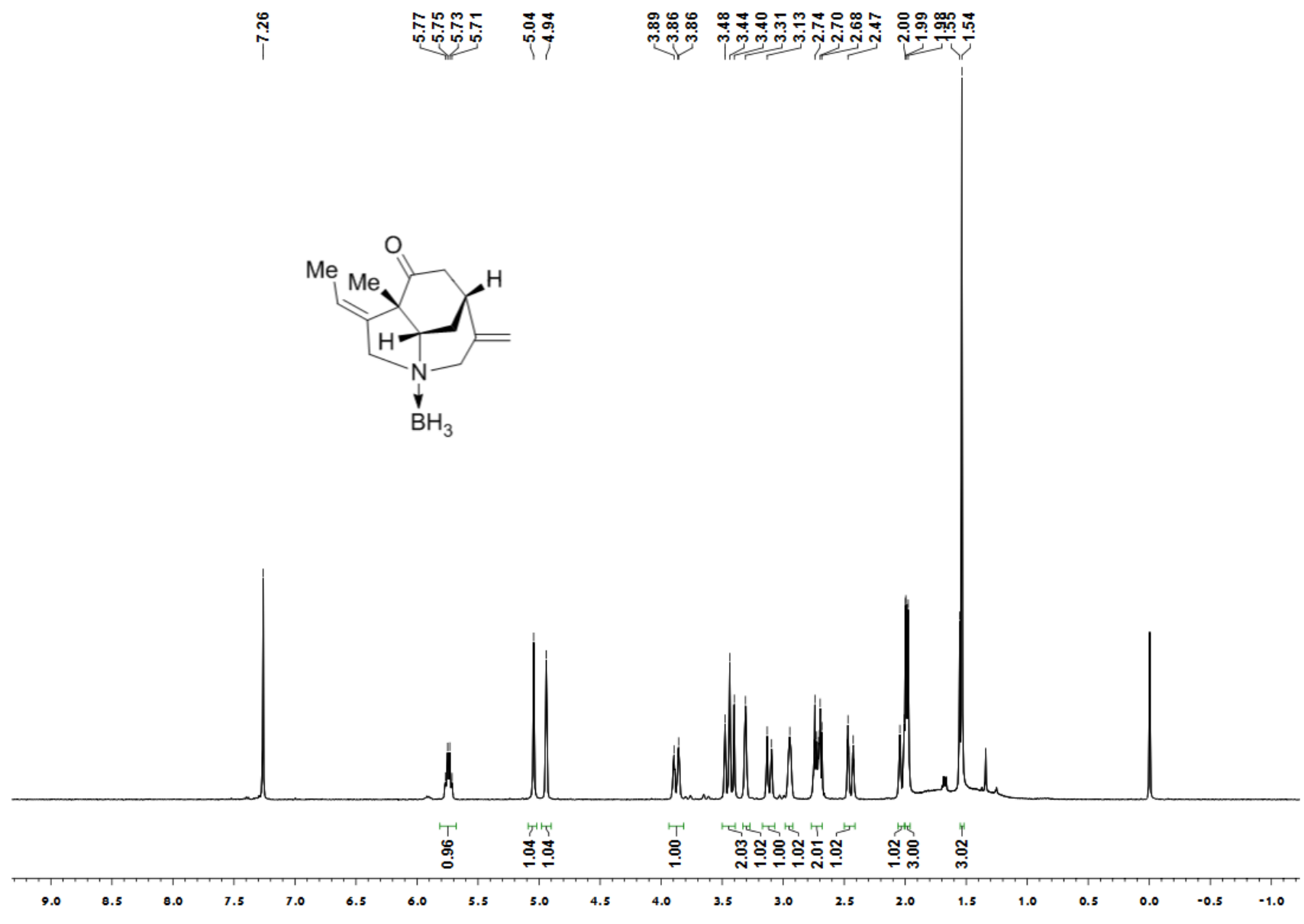

${ }^{13} \mathrm{C}$ NMR Spectra of $23\left(101 \mathrm{MHz}, \mathrm{CDCl}_{3}\right)$

\begin{tabular}{|c|c|c|c|c|c|c|c|}
\hline 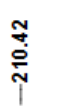 & 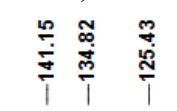 & $\stackrel{\bar{p}}{\stackrel{\bar{p}}{i}}$ & 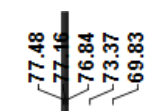 & 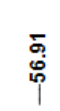 & 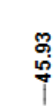 & & $\begin{array}{l}\text { : } \\
\stackrel{0}{0}\end{array}$ \\
\hline
\end{tabular}

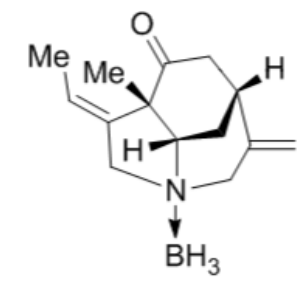

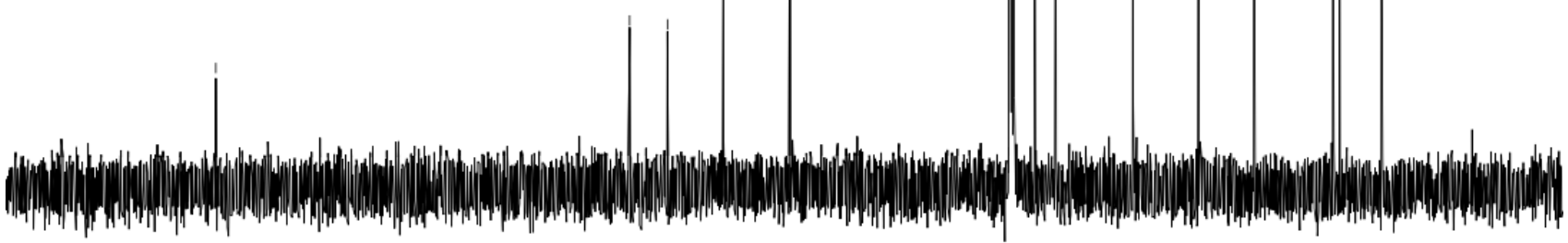

$\begin{array}{llllllllllllllllllllllllll}240 & 230 & 220 & 210 & 200 & 190 & 180 & 170 & 160 & 150 & 140 & 130 & 120 & 110 & 100 & 90 & 80 & 70 & 60 & 50 & 40 & 30 & 20 & 10 & 0 & -10\end{array}$ 
${ }^{1} \mathrm{H}$ NMR Spectra of $24\left(400 \mathrm{MHz}, \mathrm{CDCl}_{3}\right)$

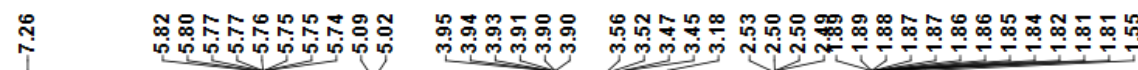

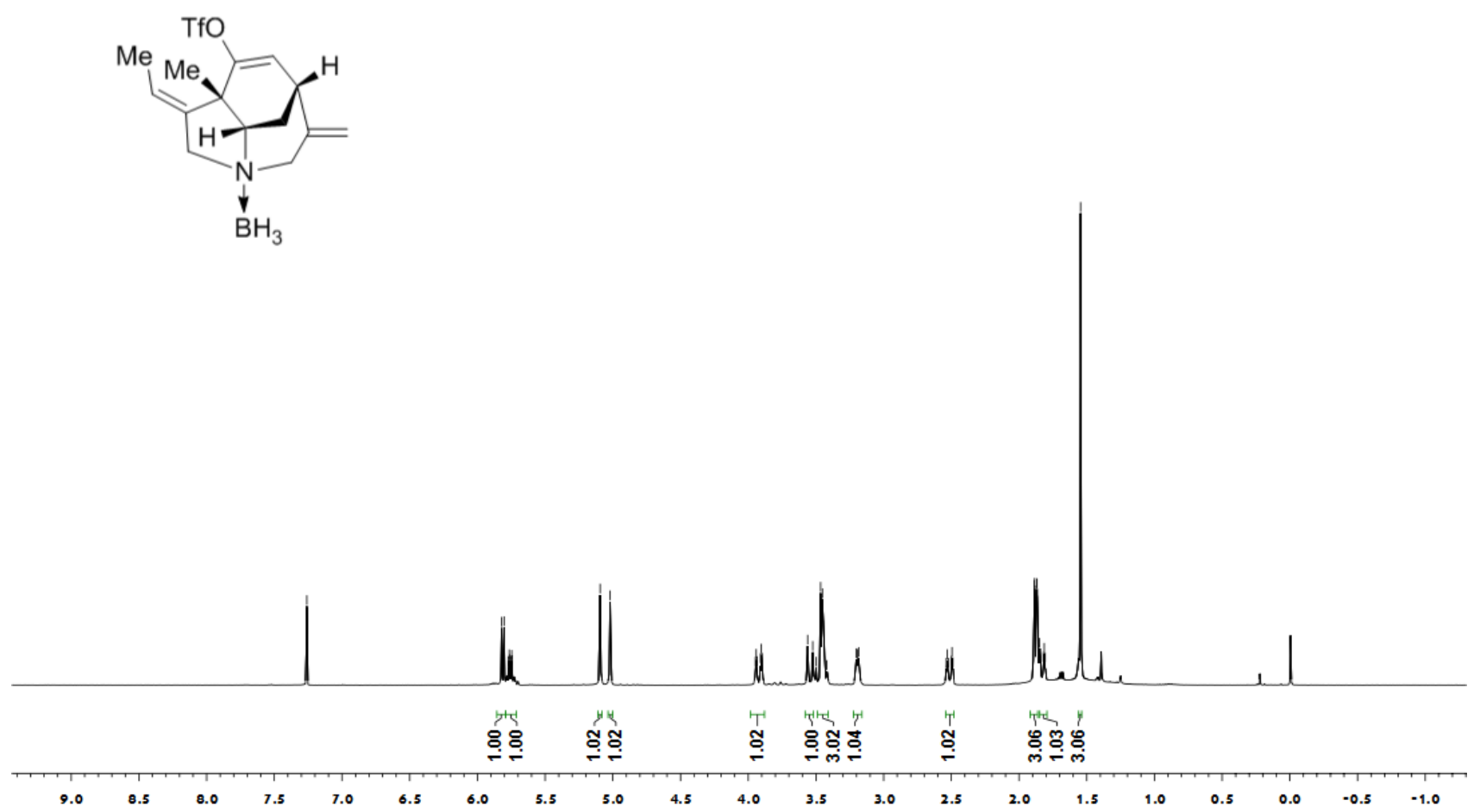

${ }^{13} \mathrm{C}$ NMR Spectra of $24\left(101 \mathrm{MHz}, \mathrm{CDCl}_{3}\right)$

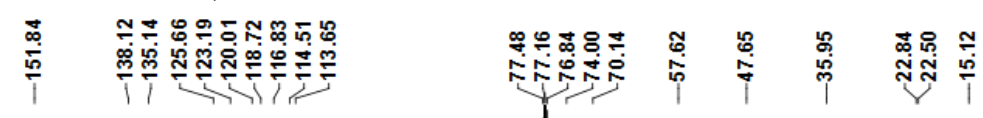
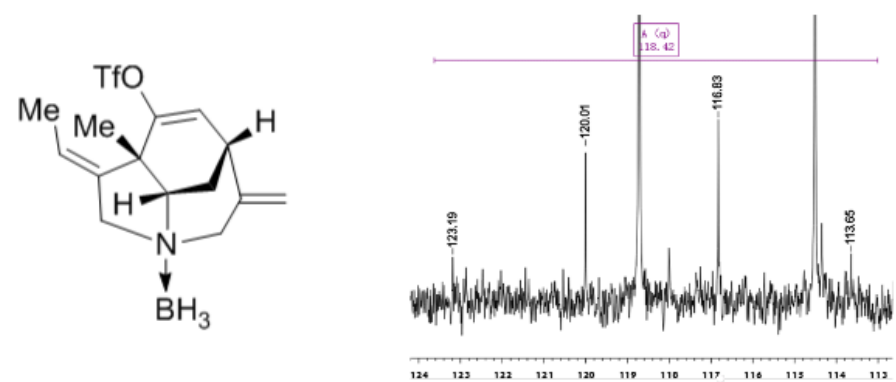

$\begin{array}{llllllllllllllllllllllll}220 & 210 & 200 & 190 & 180 & 170 & 160 & 150 & 140 & 130 & 120 & 110 & 100 & 90 & 80 & 70 & 60 & 50 & 40 & 30 & 20 & 10 & 0 & -10\end{array}$ 
${ }^{1} \mathrm{H}$ NMR Spectra of $25\left(400 \mathrm{MHz}, \mathrm{CDCl}_{3}\right)$

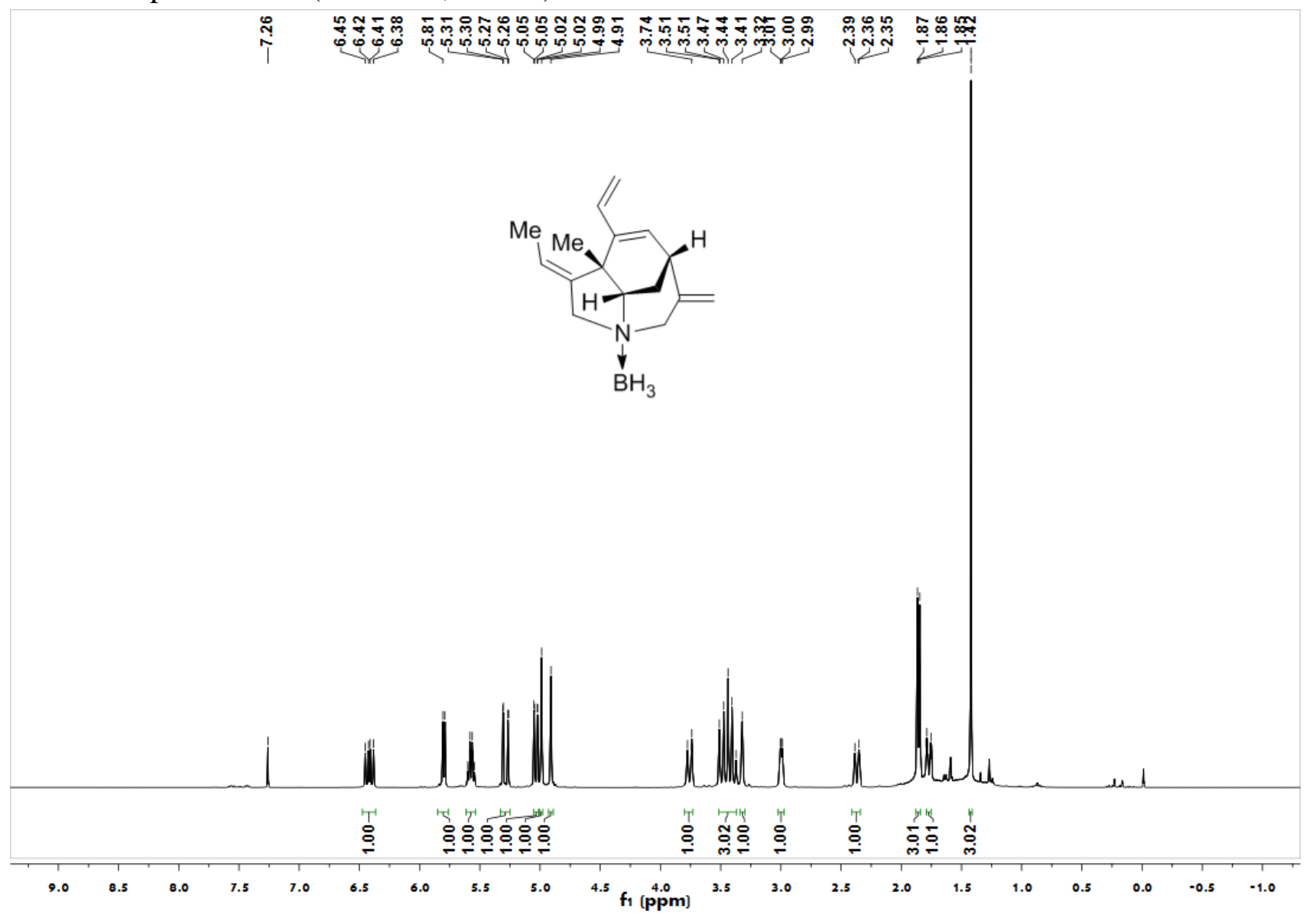

${ }^{13} \mathrm{C}$ NMR Spectra of 25 (101 MHz, $\left.\mathrm{CDCl}_{3}\right)$

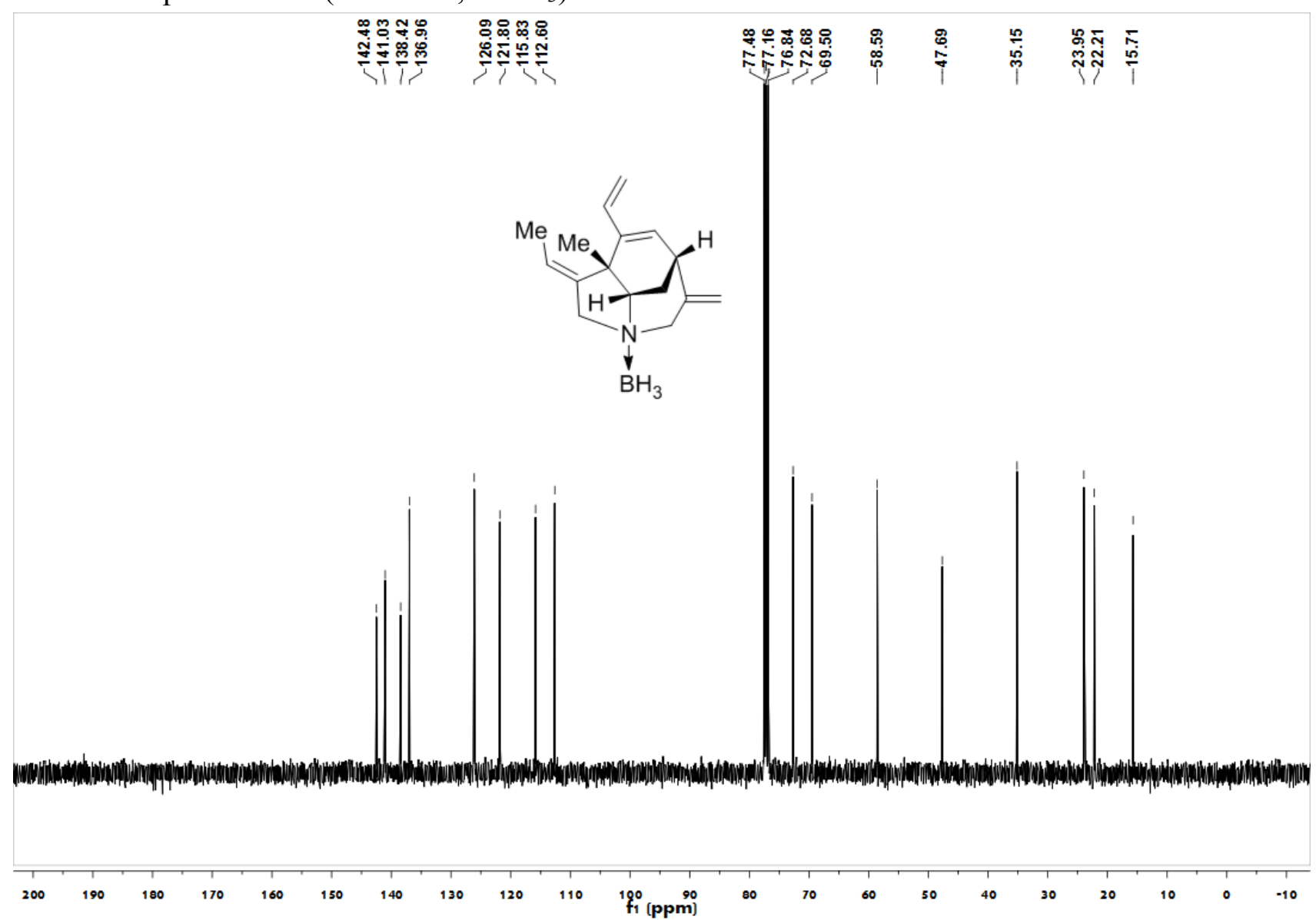


${ }^{1} \mathrm{H}$ NMR Spectra of $26\left(400 \mathrm{MHz}, \mathrm{CDCl}_{3}\right)$

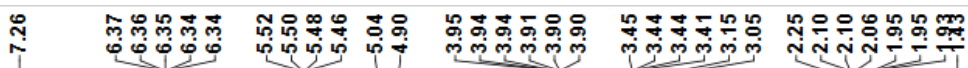

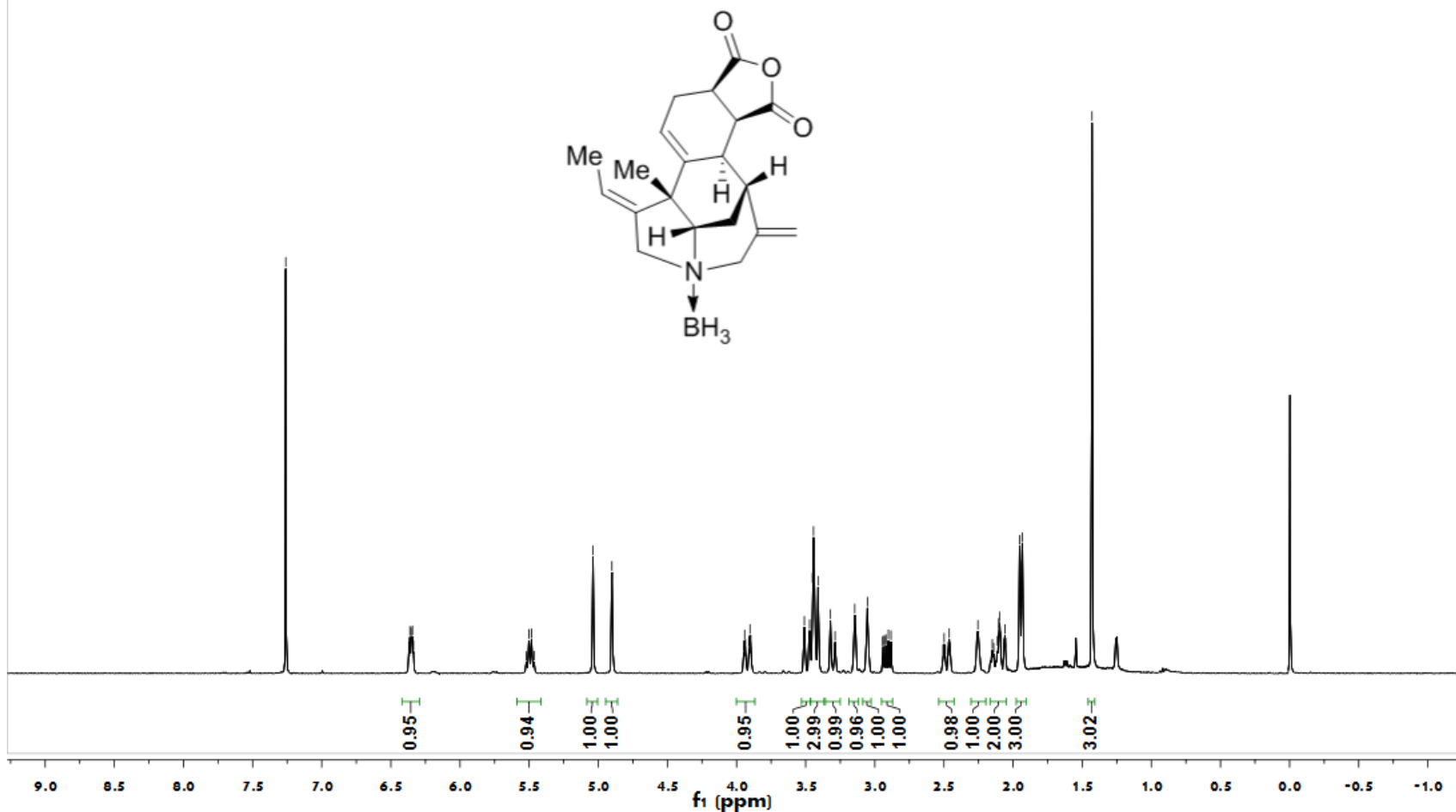

${ }^{13} \mathrm{C}$ NMR Spectra of $26\left(101 \mathrm{MHz}, \mathrm{CDCl}_{3}\right)$

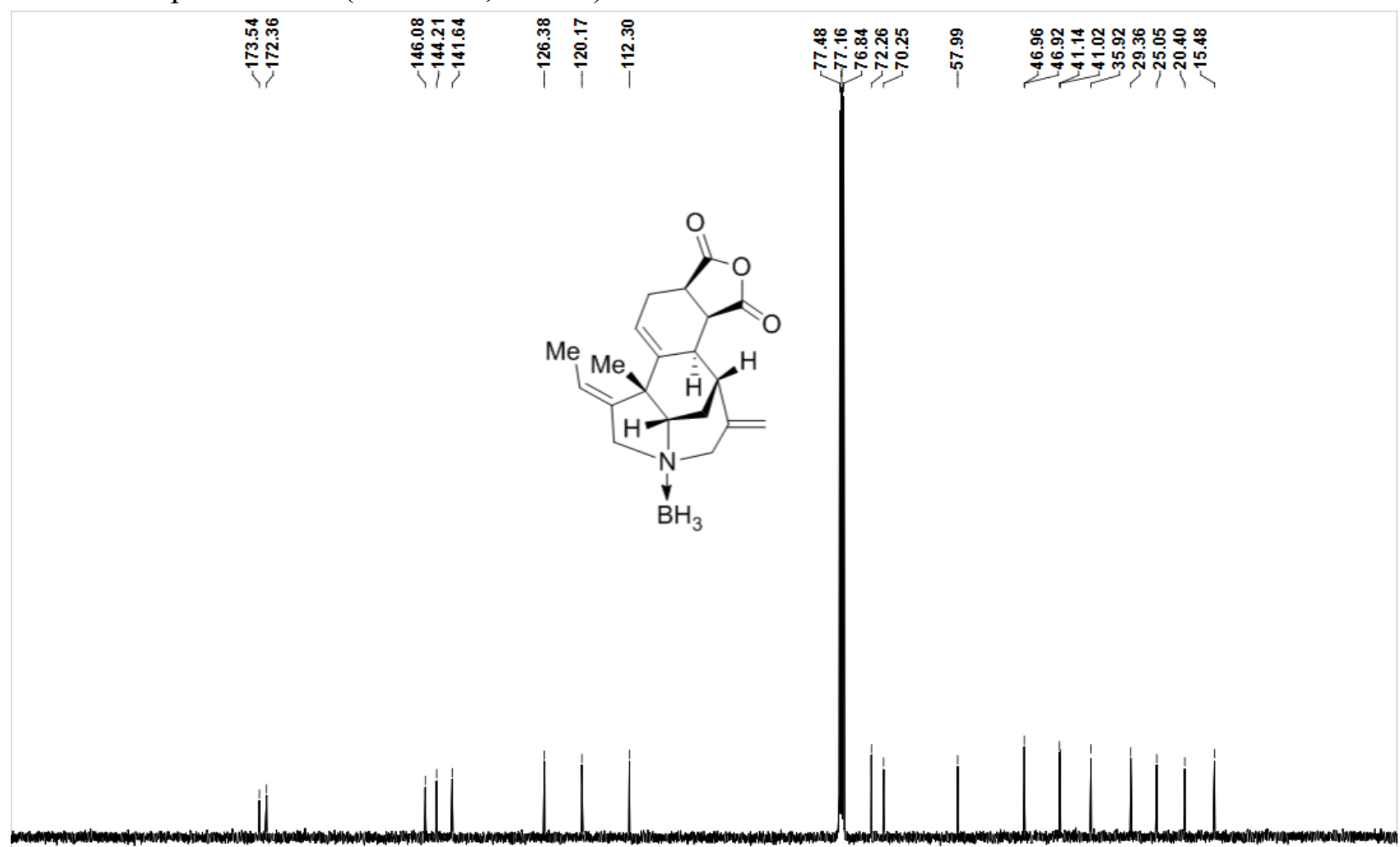

$\begin{array}{llllllllllllllllllllllll}210 & 200 & 190 & 180 & 170 & 160 & 150 & 140 & 130 & 120 & 110 & \mathrm{f}_{1}\left(\begin{array}{ll}100 \\ \text { (ppm) }\end{array}\right. & 90 & 80 & 70 & 60 & 50 & 40 & 30 & 20 & 10 & 0 & -10\end{array}$ 
${ }^{1} \mathrm{H}$ NMR Spectra of $27\left(400 \mathrm{MHz}, \mathrm{CDCl}_{3}\right)$

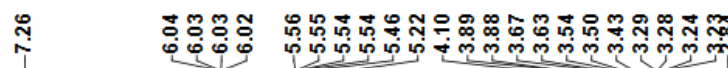

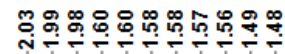
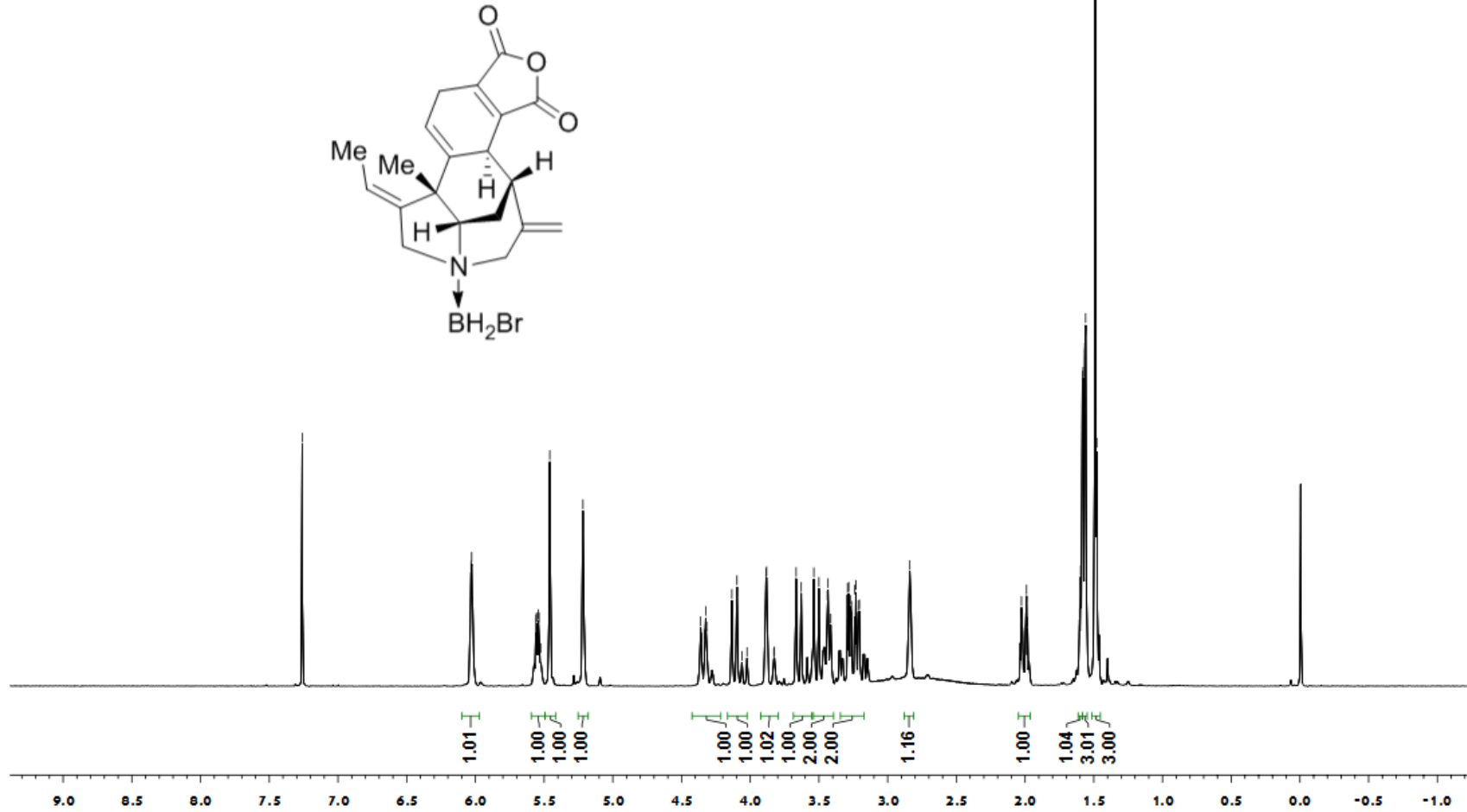

${ }^{13} \mathrm{C}$ NMR Spectra of $27\left(101 \mathrm{MHz}, \mathrm{CDCl}_{3}\right)$

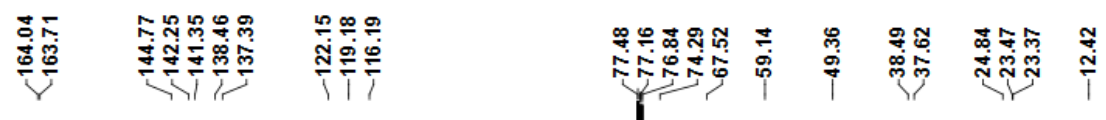

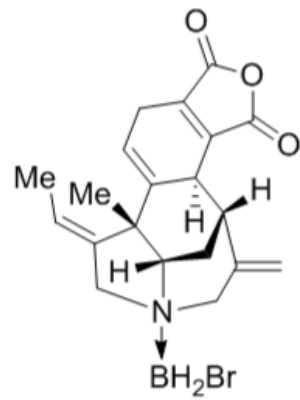

$\begin{array}{lllllllllllllll}220 & 210 & 200 & 190 & 180 & 170 & 160 & 150 & 140 & 130 & 120 & 110 & 100 & 90 & 80\end{array}$

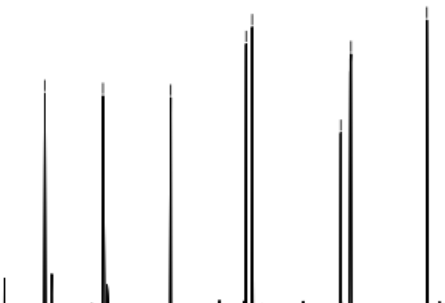


${ }^{1} \mathrm{H}$ NMR Spectra of $28\left(400 \mathrm{MHz}, \mathrm{CDCl}_{3}\right)$

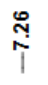

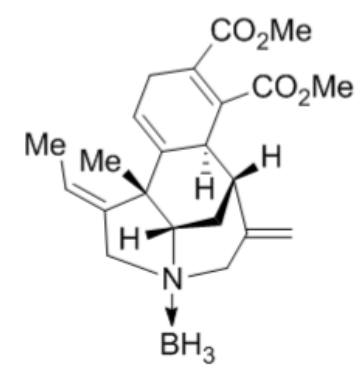

${ }^{13} \mathrm{C}$ NMR Spectra of $28\left(101 \mathrm{MHz}, \mathrm{CDCl}_{3}\right)$

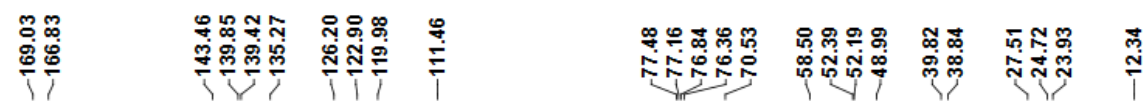
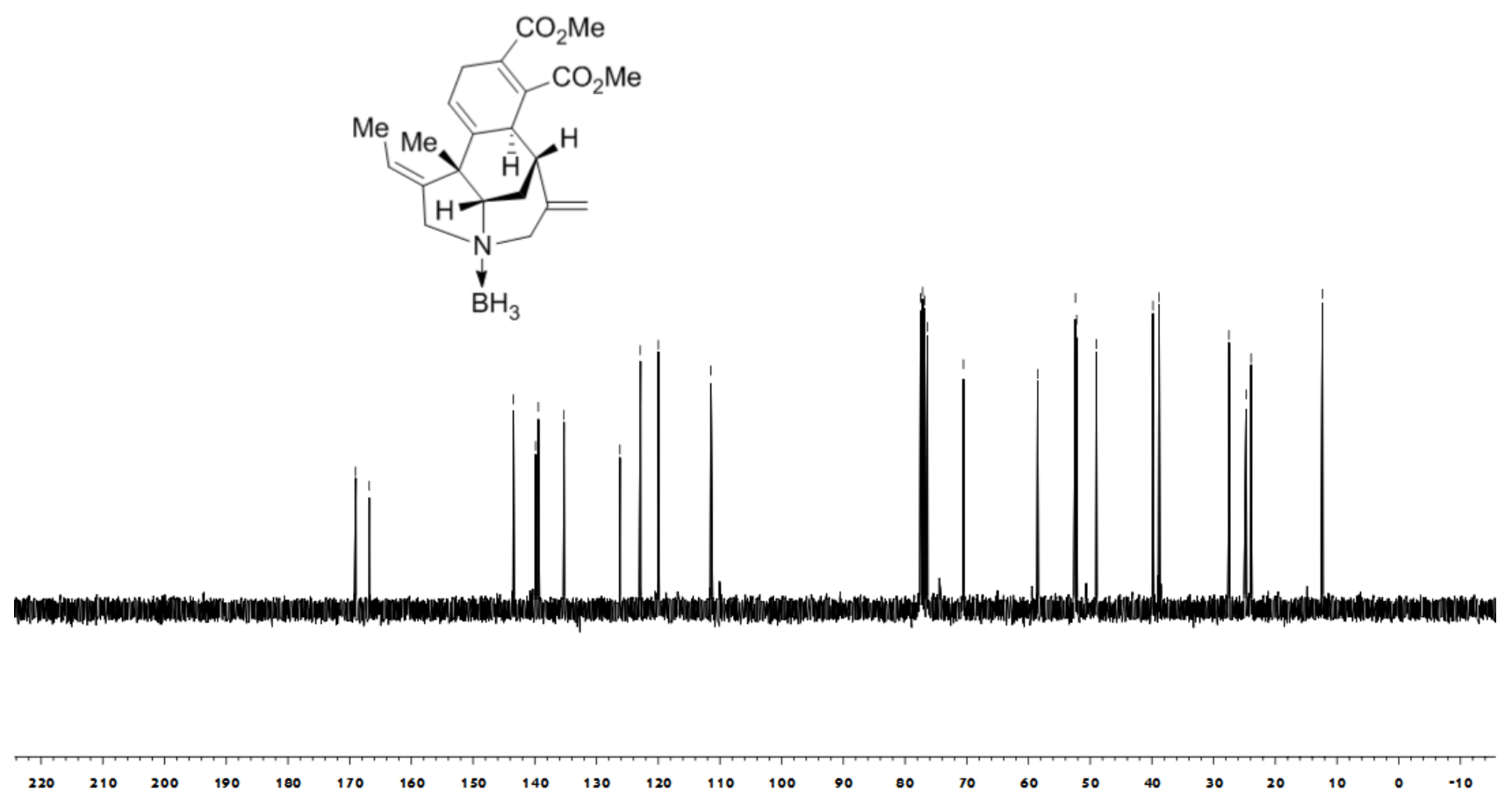
${ }^{1} \mathrm{H}$ NMR Spectra of $29\left(400 \mathrm{MHz}, \mathrm{CDCl}_{3}\right)$

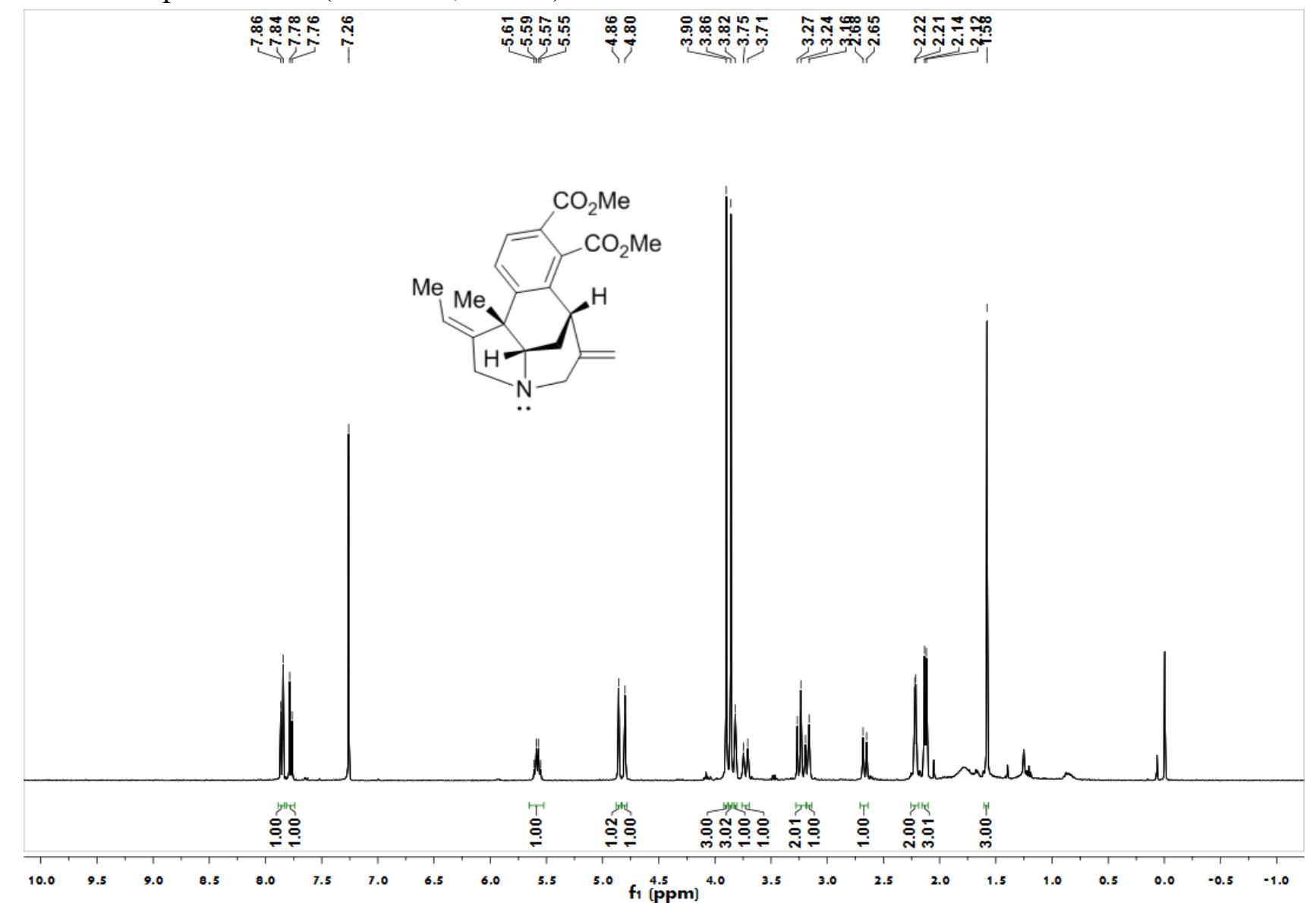

${ }^{13} \mathrm{C}$ NMR Spectra of $29\left(101 \mathrm{MHz}, \mathrm{CDCl}_{3}\right)$

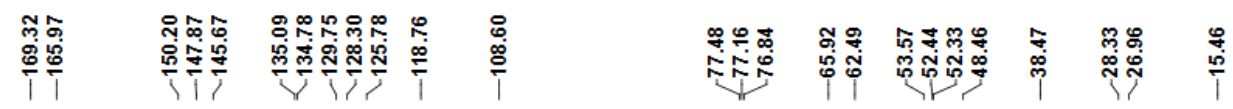
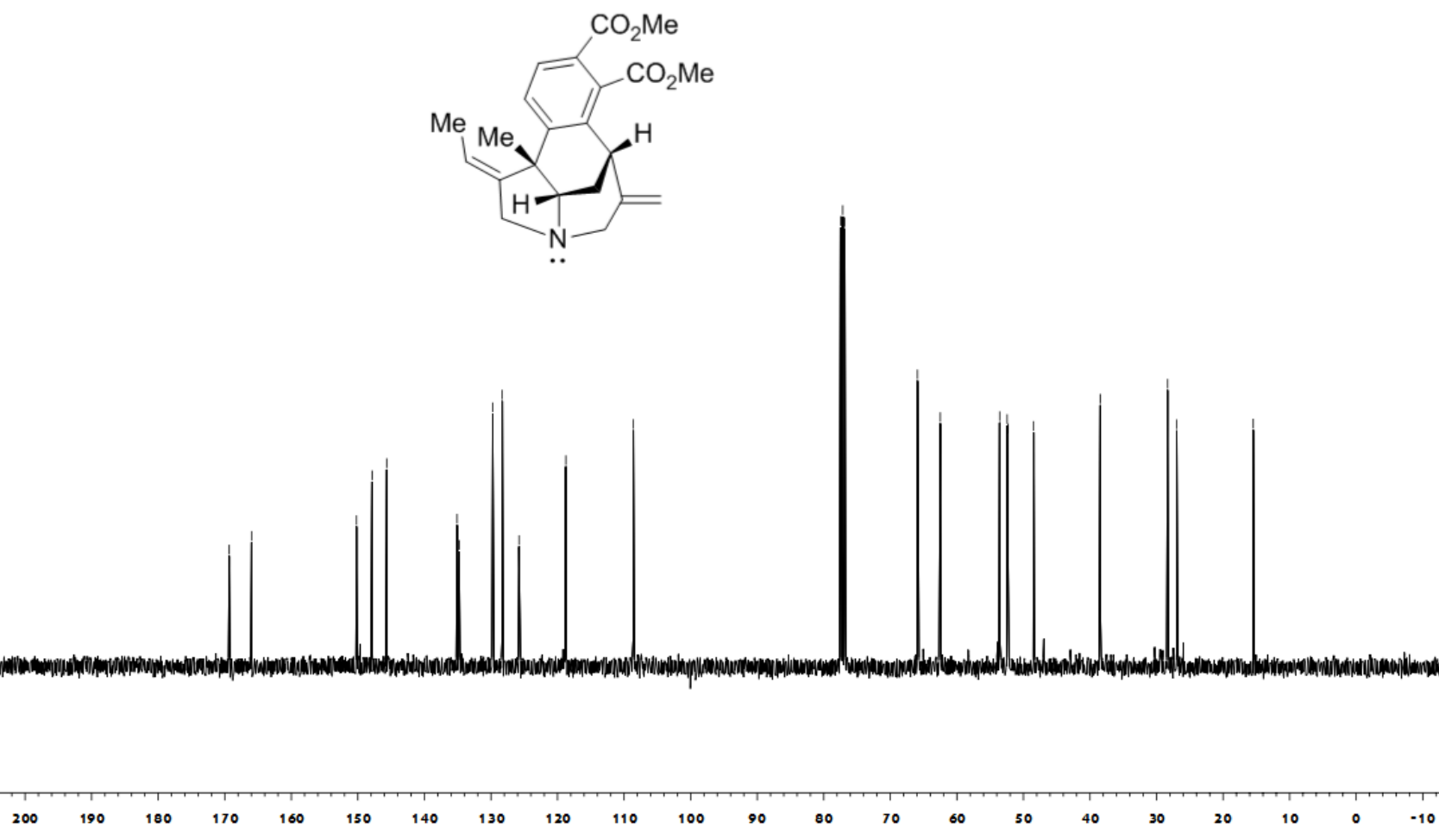
${ }^{1} \mathrm{H}$ NMR Spectra of $\mathbf{3 0}\left(400 \mathrm{MHz}, \mathrm{CDCl}_{3}\right)$

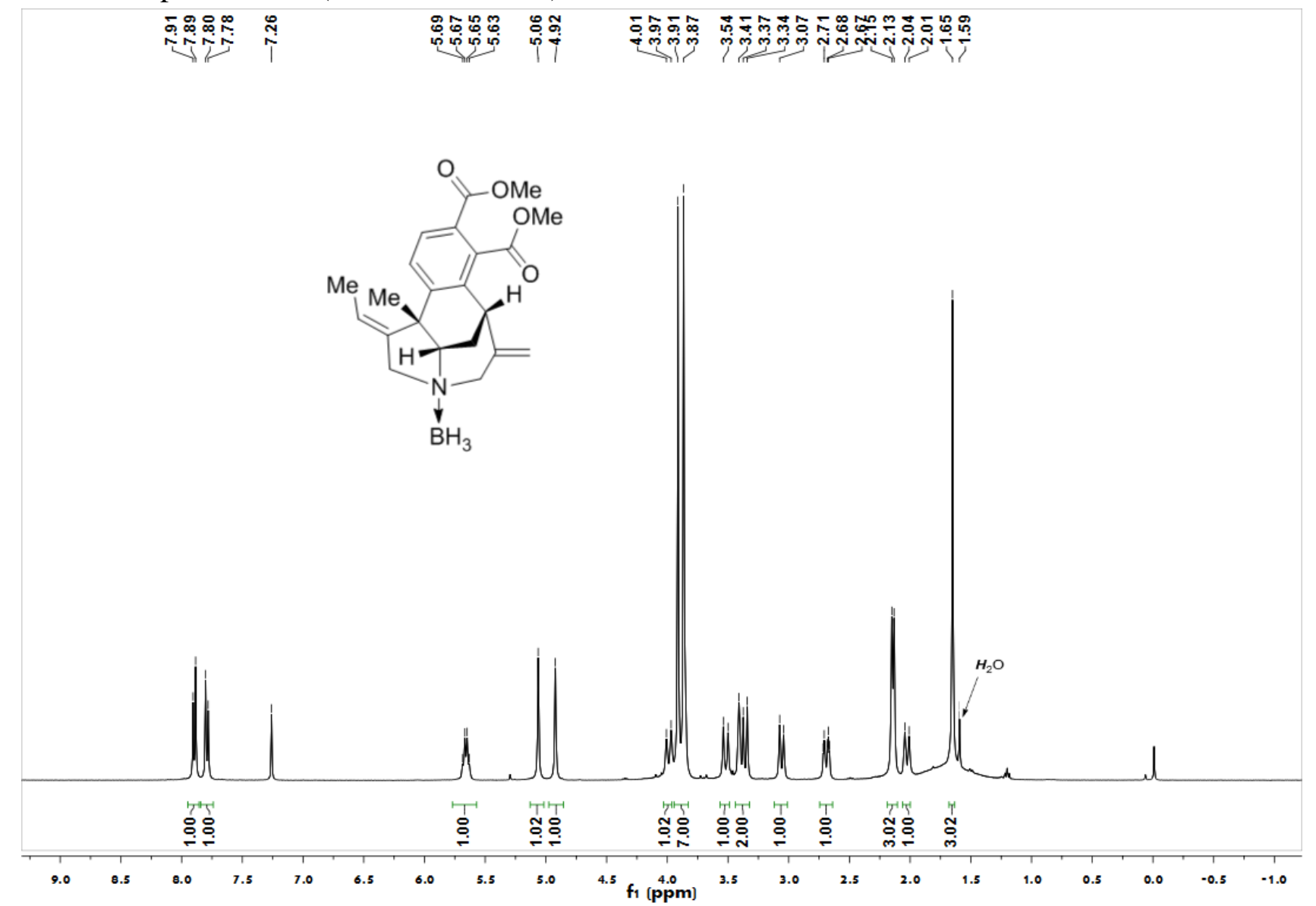

${ }^{13} \mathrm{C}$ NMR Spectra of $\mathbf{3 0}\left(101 \mathrm{MHz}, \mathrm{CDCl}_{3}\right)$

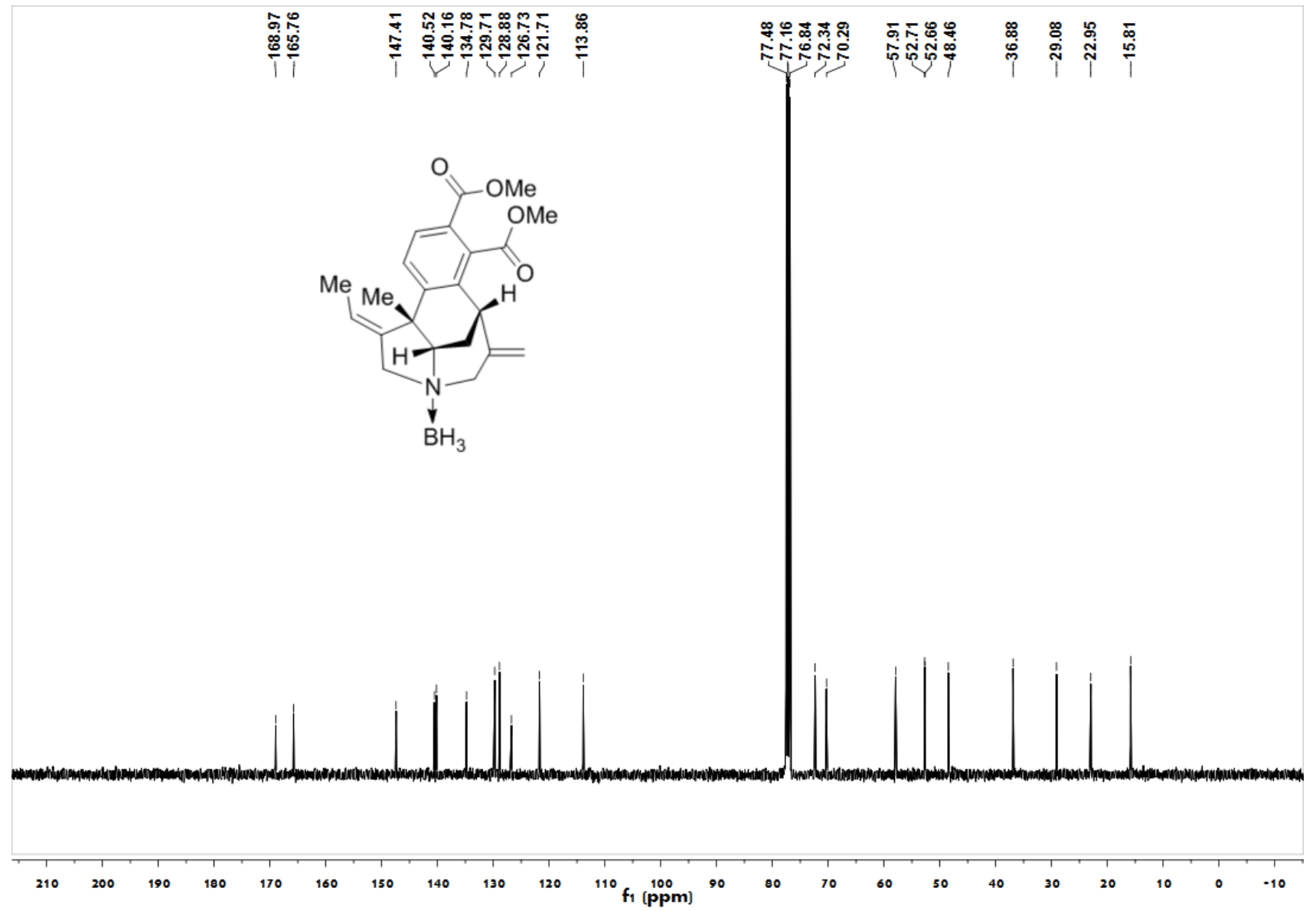




\section{III ${ }^{11} B$ NMR Spectra of Compounds}

${ }^{11} \mathrm{~B}$ NMR Spectra of $23\left(128 \mathrm{MHz}, \mathrm{CDCl}_{3}\right)$
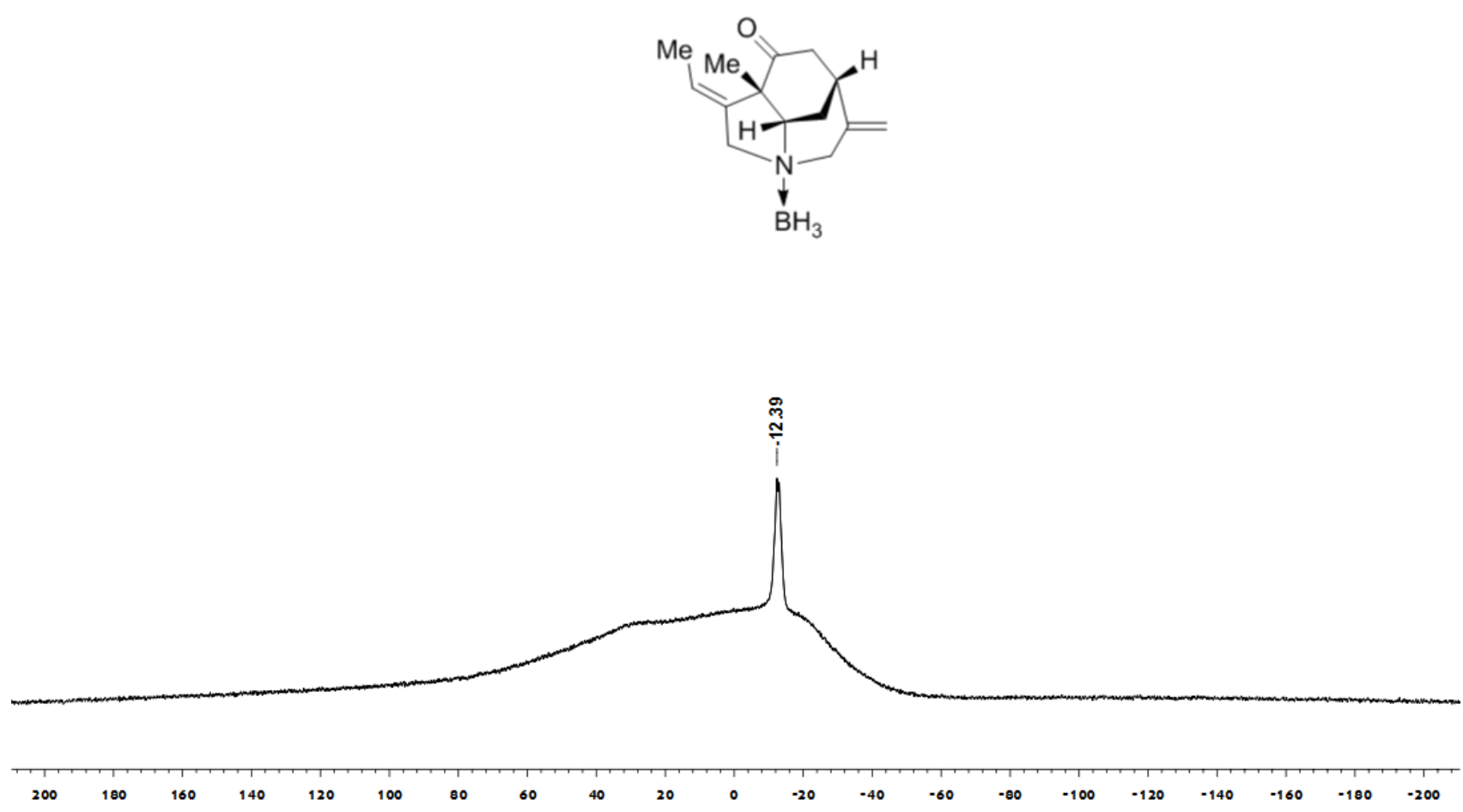

${ }^{11} \mathrm{~B}$ NMR Spectra of $24\left(128 \mathrm{MHz}, \mathrm{CDCl}_{3}\right)$

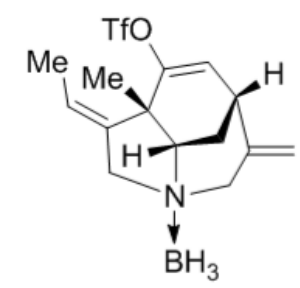


${ }^{11} \mathrm{~B}$ NMR Spectra of $25\left(128 \mathrm{MHz}, \mathrm{CDCl}_{3}\right)$
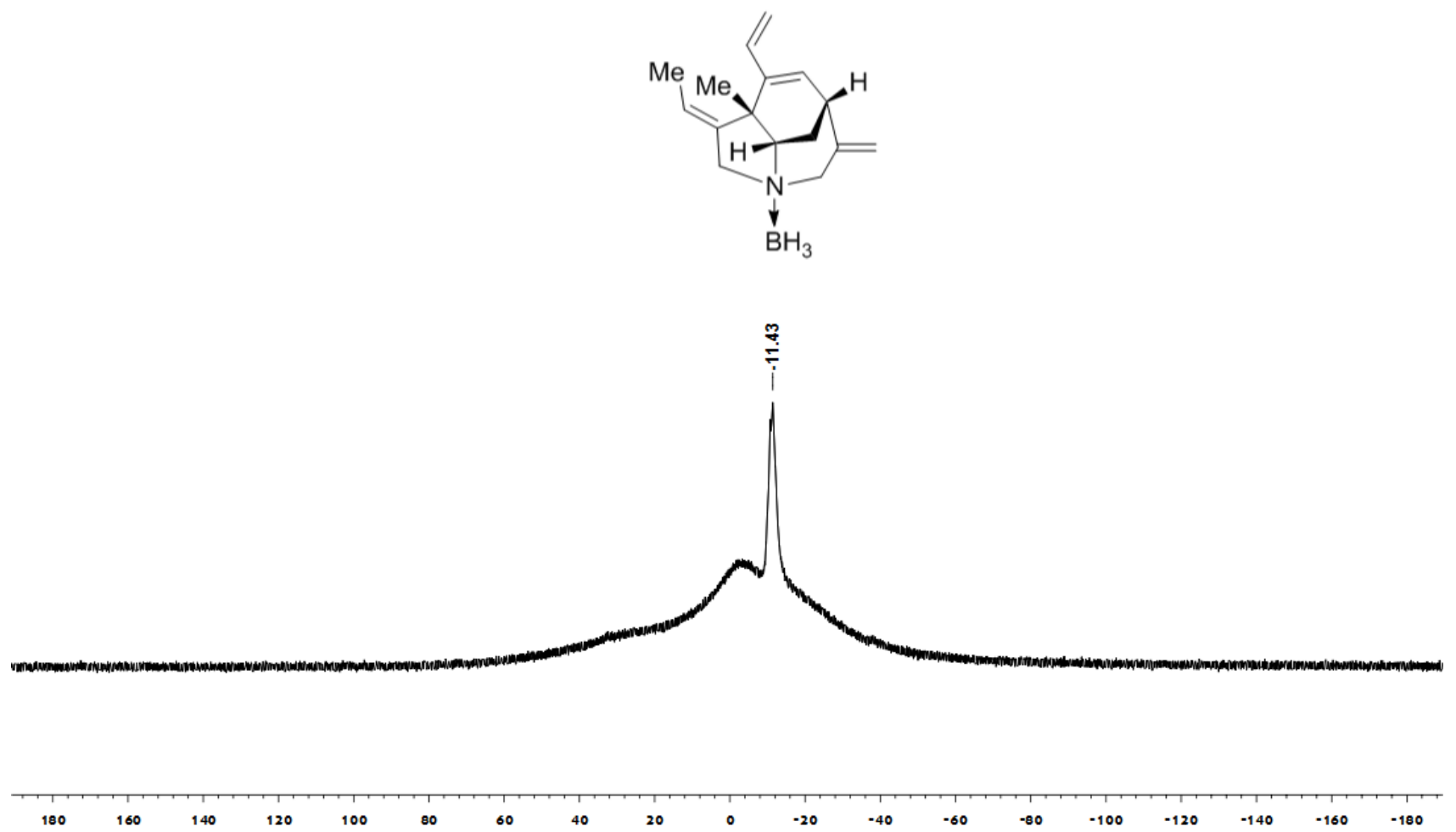

${ }^{11} \mathrm{~B}$ NMR Spectra of $26\left(128 \mathrm{MHz}, \mathrm{CDCl}_{3}\right)$
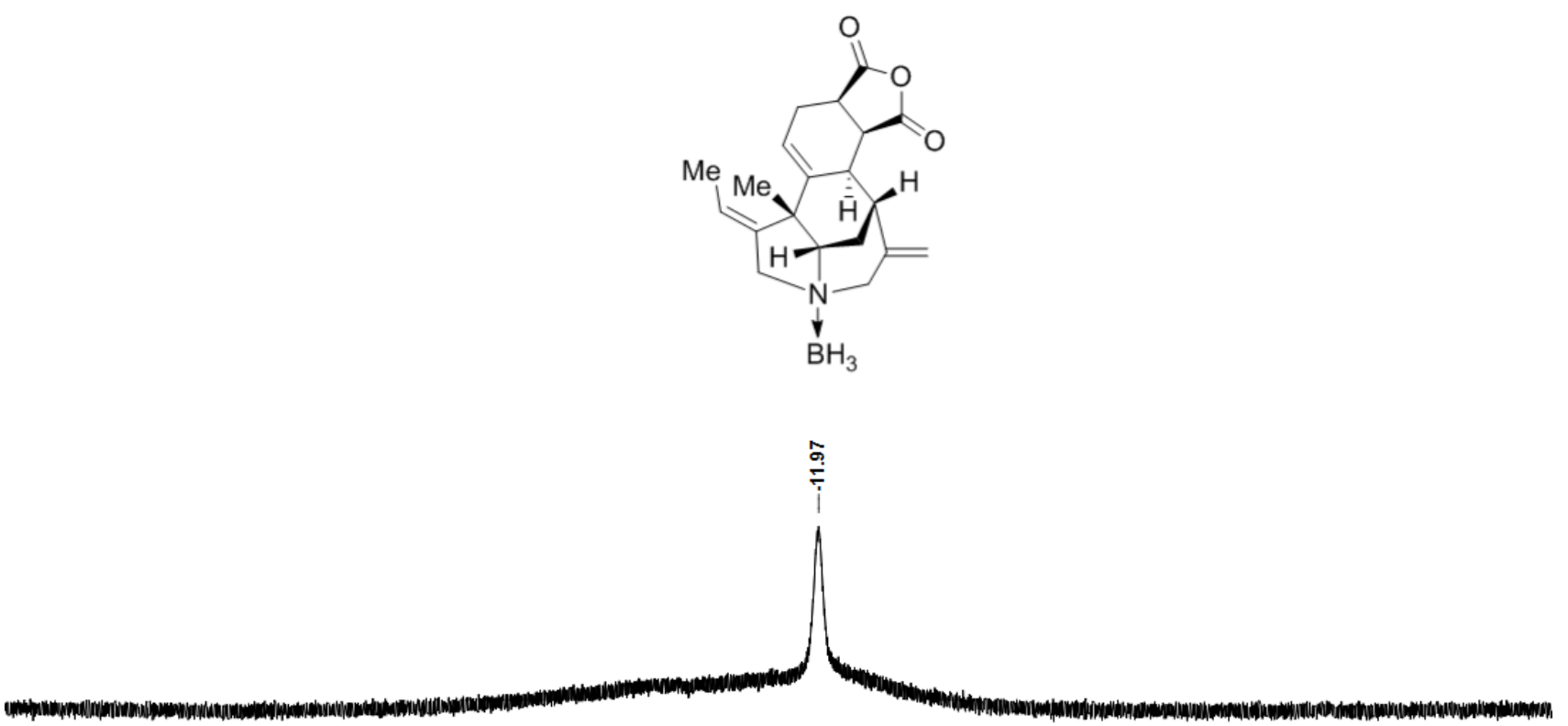
${ }^{11} \mathrm{~B}$ NMR Spectra of $27\left(128 \mathrm{MHz}, \mathrm{CDCl}_{3}\right)$
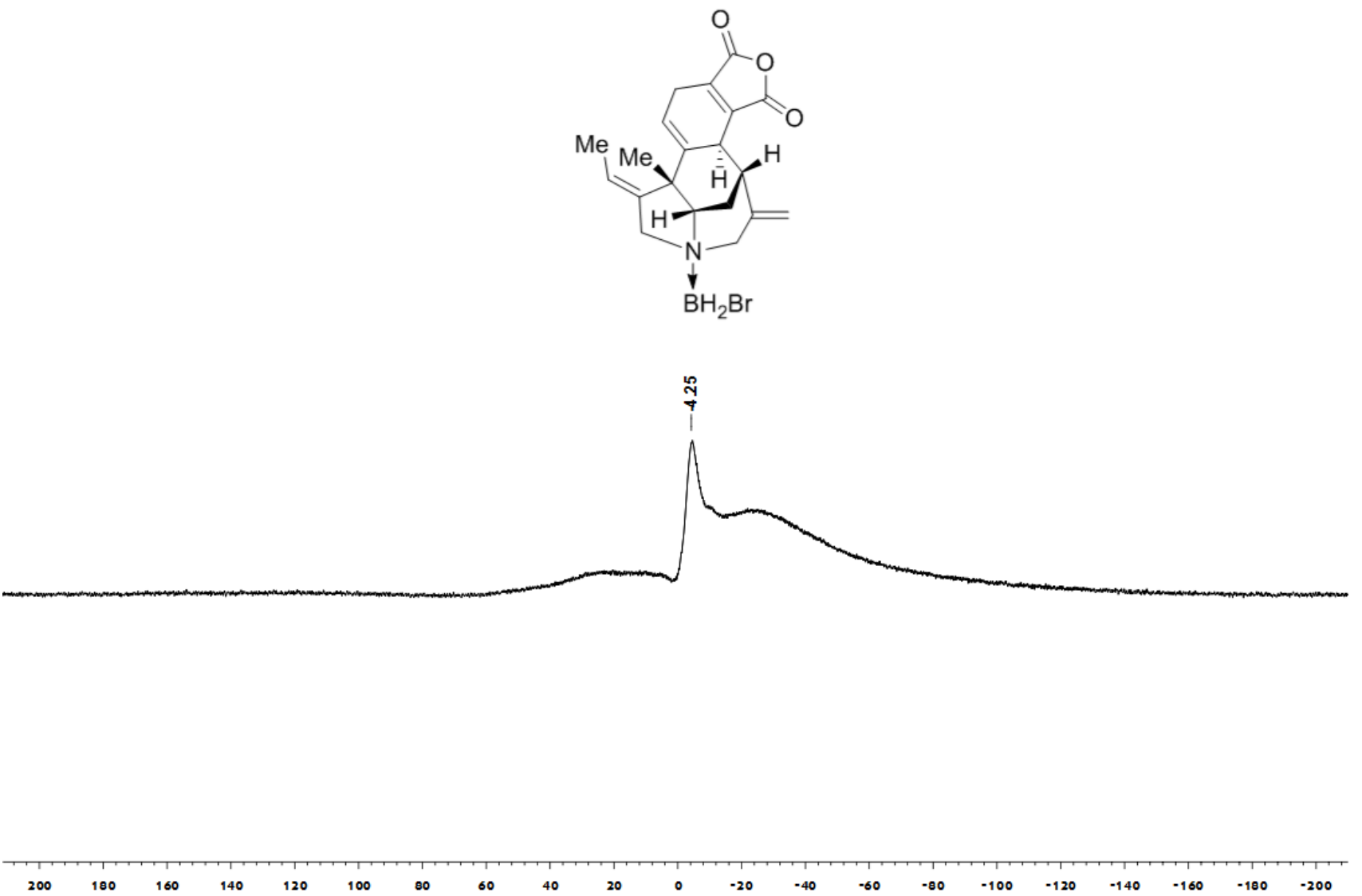

${ }^{11} \mathrm{~B}$ NMR Spectra of $28\left(128 \mathrm{MHz}, \mathrm{CDCl}_{3}\right)$
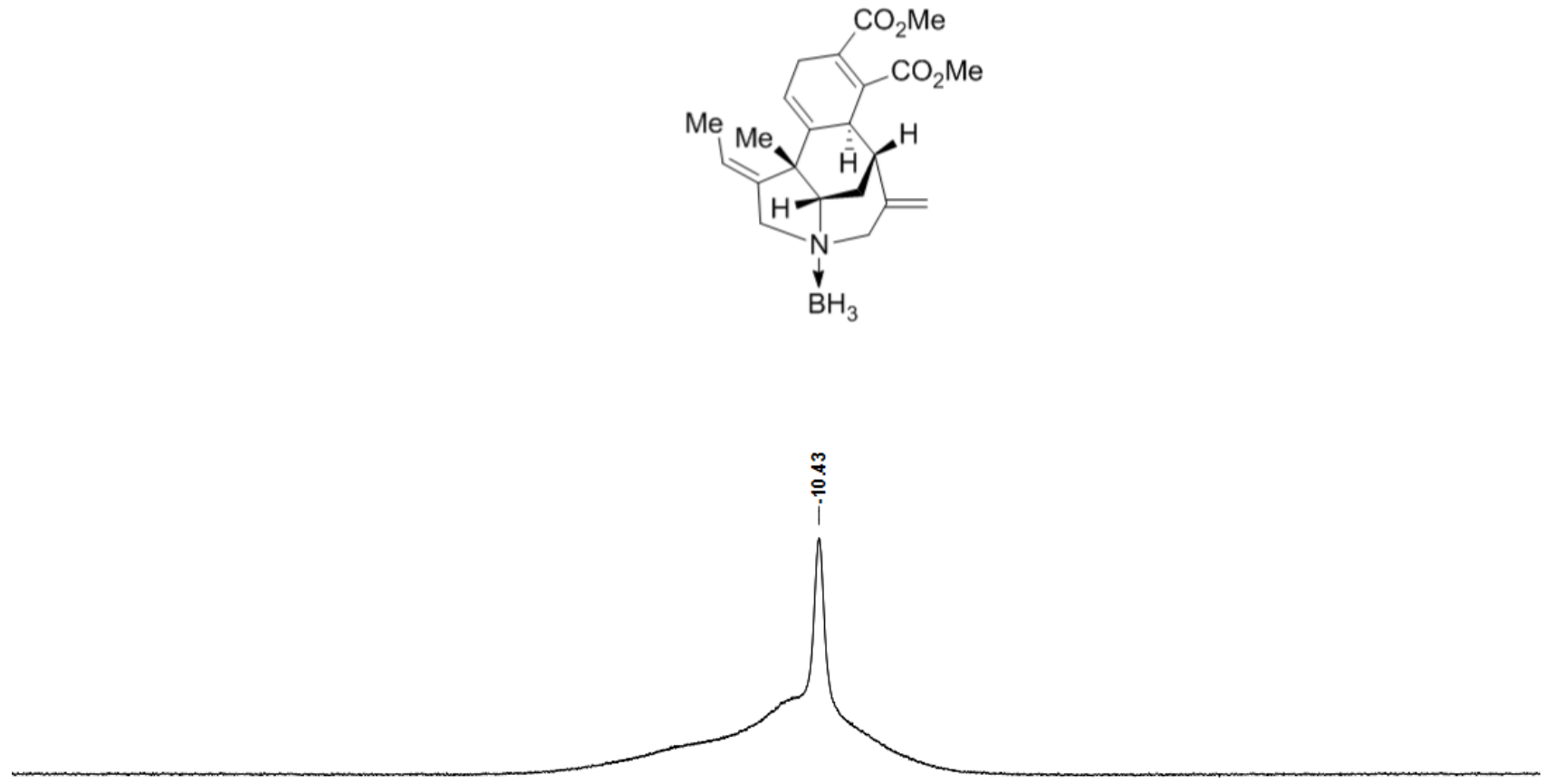

$\begin{array}{lllllllllllllllllllll}200 & 180 & 160 & 140 & 120 & 100 & 80 & 60 & 40 & 20 & 0 & -20 & -40 & -60 & -80 & -100 & -120 & -140 & -160 & -180 & -200\end{array}$ 
${ }^{11} \mathrm{~B}$ NMR Spectra of $\mathbf{3 0}\left(128 \mathrm{MHz}, \mathrm{CDCl}_{3}\right)$
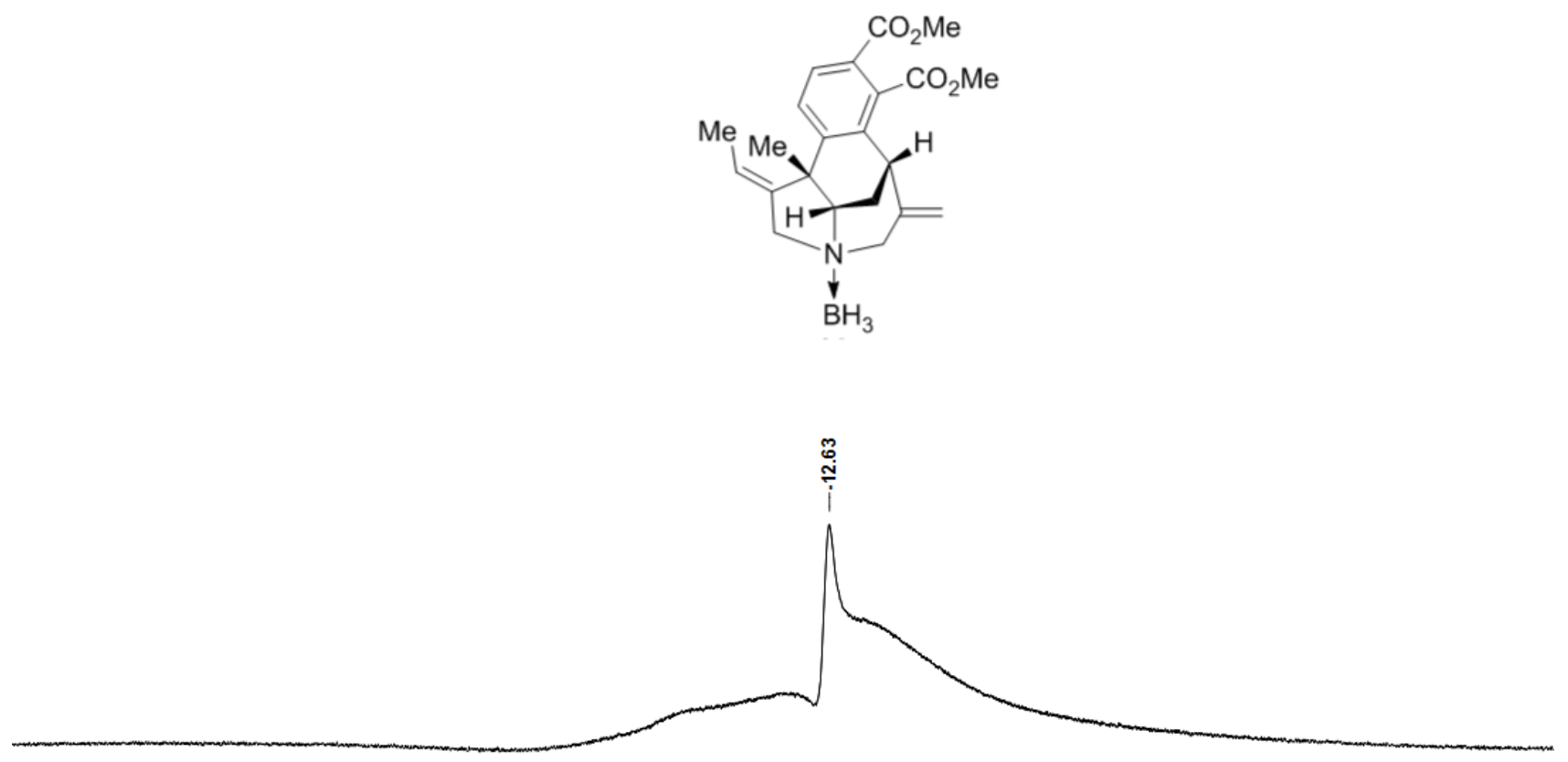

$\begin{array}{lllllllllllllllllllll}200 & 180 & 160 & 140 & 120 & 100 & 80 & 60 & 40 & 20 & 0 & -20 & -40 & -60 & -80 & -100 & -120 & -140 & -160 & -180 & -200\end{array}$ 


\section{ORTEP Drawings and Crystallographic Data of Compounds}

ORTEP Drawing and Crystallographic Data of Compound 17 (CCDC 1893589)

io
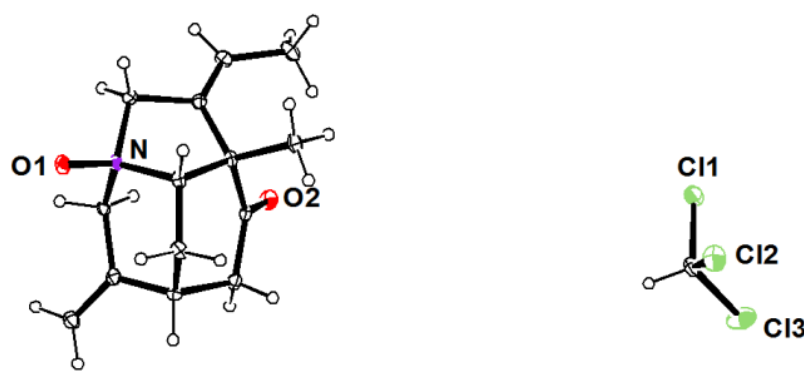

\begin{tabular}{|c|c|}
\hline Identification code & tertamineoxide \\
\hline Empirical formula & $\mathrm{C}_{15} \mathrm{H}_{22} \mathrm{NO}_{3} \mathrm{Cl}_{3}$ \\
\hline Formula weight & 370.70 \\
\hline Temperature/K & 113.15 \\
\hline Crystal system & monoclinic \\
\hline Space group & $\mathrm{I} 2$ \\
\hline $\mathrm{a} / \AA$ & $12.1610(2)$ \\
\hline $\mathrm{b} / \AA$ & $5.97494(11)$ \\
\hline $\mathrm{c} / \AA$ & $23.8401(4)$ \\
\hline$\alpha /^{\circ}$ & 90 \\
\hline$\beta /{ }^{\circ}$ & $97.7205(15)$ \\
\hline$\gamma /{ }^{\circ}$ & 90 \\
\hline Volume $/ \AA^{3}$ & $1716.55(5)$ \\
\hline $\mathrm{Z}$ & 2 \\
\hline$\rho_{\text {calc }} \mathrm{g} / \mathrm{cm}^{3}$ & 1.398 \\
\hline$\mu / \mathrm{mm}^{-1}$ & 0.541 \\
\hline $\mathrm{F}(000)$ & 754.0 \\
\hline Crystal size $/ \mathrm{mm}^{3}$ & $0.2 \times 0.18 \times 0.12$ \\
\hline Radiation & $\operatorname{MoK} \alpha(\lambda=0.71073)$ \\
\hline $2 \Theta$ range for data collection $/{ }^{\circ}$ & 3.448 to 54.954 \\
\hline Index ranges & $-15 \leq \mathrm{h} \leq 15,-7 \leq \mathrm{k} \leq 7,-30 \leq 1 \leq 30$ \\
\hline Reflections collected & 10393 \\
\hline Independent reflections & $3888\left[\mathrm{R}_{\mathrm{int}}=0.0123, \mathrm{R}_{\text {sigma }}=0.0112\right]$ \\
\hline Data/restraints/parameters & $3888 / 1 / 200$ \\
\hline Goodness-of-fit on $\mathrm{F}^{2}$ & 1.069 \\
\hline Final $\mathrm{R}$ indexes $[\mathrm{I}>=2 \sigma(\mathrm{I})]$ & $\mathrm{R}_{1}=0.0235, \mathrm{wR}_{2}=0.0600$ \\
\hline Final $\mathrm{R}$ indexes [all data] & $\mathrm{R}_{1}=0.0240, \mathrm{w} \mathrm{R}_{2}=0.0602$ \\
\hline Largest diff. peak/hole / e $\AA^{-3}$ & $0.29 /-0.23$ \\
\hline Flack parameter & $0.009(11)$ \\
\hline
\end{tabular}


ORTEP Drawing and Crystallographic Data of Compound 23 (CCDC 1893590)

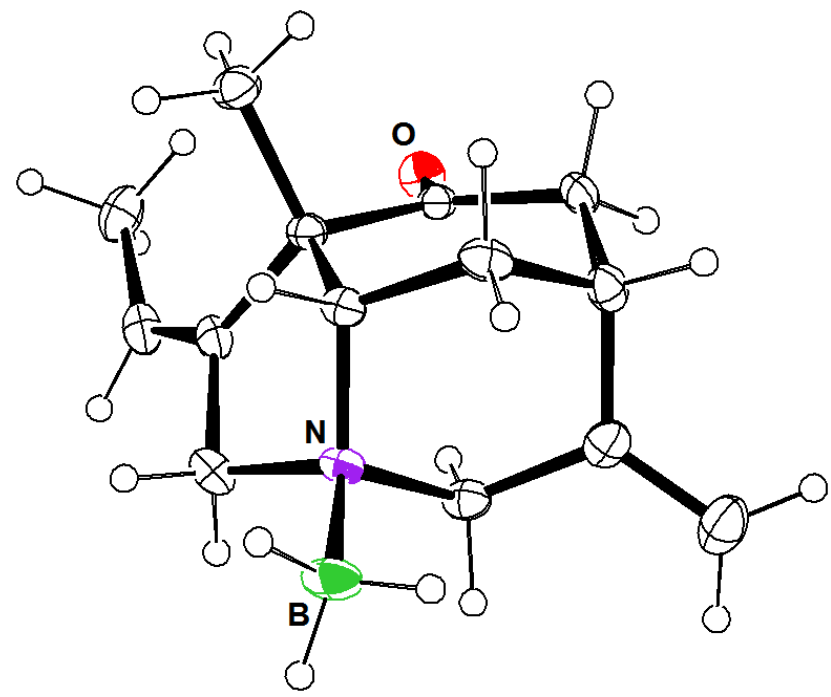

\begin{tabular}{|c|c|}
\hline Identification code & tertamineborane \\
\hline Empirical formula & $\mathrm{C}_{14} \mathrm{H}_{22} \mathrm{BNO}$ \\
\hline Formula weight & 231.13 \\
\hline Temperature/K & 113.15 \\
\hline Crystal system & monoclinic \\
\hline Space group & $\mathrm{P} 21$ \\
\hline $\mathrm{a} / \AA$ & $8.9980(18)$ \\
\hline $\mathrm{b} / \AA$ & $7.2087(14)$ \\
\hline $\mathrm{c} / \AA$ & $11.219(2)$ \\
\hline$\alpha /^{\circ}$ & 90 \\
\hline$\beta /{ }^{\circ}$ & $111.51(3)$ \\
\hline$\gamma /{ }^{\circ}$ & 90 \\
\hline Volume/Å3 & $677.0(3)$ \\
\hline $\mathrm{Z}$ & 2 \\
\hline$\rho c a l c g / \mathrm{cm} 3$ & 1.134 \\
\hline$\mu / \mathrm{mm}-1$ & 0.069 \\
\hline $\mathrm{F}(000)$ & 252.0 \\
\hline Crystal size/mm3 & $0.2 \times 0.18 \times 0.12$ \\
\hline Radiation & $\operatorname{MoK} \alpha(\lambda=0.71073)$ \\
\hline $2 \Theta$ range for data collection $/^{\circ}$ & 7.272 to 55.622 \\
\hline Index ranges & $-11 \leq \mathrm{h} \leq 11,-9 \leq \mathrm{k} \leq 9,-14 \leq 1 \leq 14$ \\
\hline Reflections collected & 8031 \\
\hline Independent reflections & $3149[$ Rint $=0.0505$, Rsigma $=0.0774]$ \\
\hline Data/restraints/parameters & $3149 / 1 / 157$ \\
\hline Goodness-of-fit on F2 & 1.032 \\
\hline Final $R$ indexes $[\mathrm{I}>=2 \sigma(\mathrm{I})]$ & $\mathrm{R} 1=0.0570, \mathrm{wR} 2=0.1362$ \\
\hline Final $\mathrm{R}$ indexes [all data] & $\mathrm{R} 1=0.0670, \mathrm{wR} 2=0.1400$ \\
\hline Largest diff. peak/hole / e $\AA-3$ & $0.38 /-0.19$ \\
\hline Flack parameter & 0.5 \\
\hline
\end{tabular}




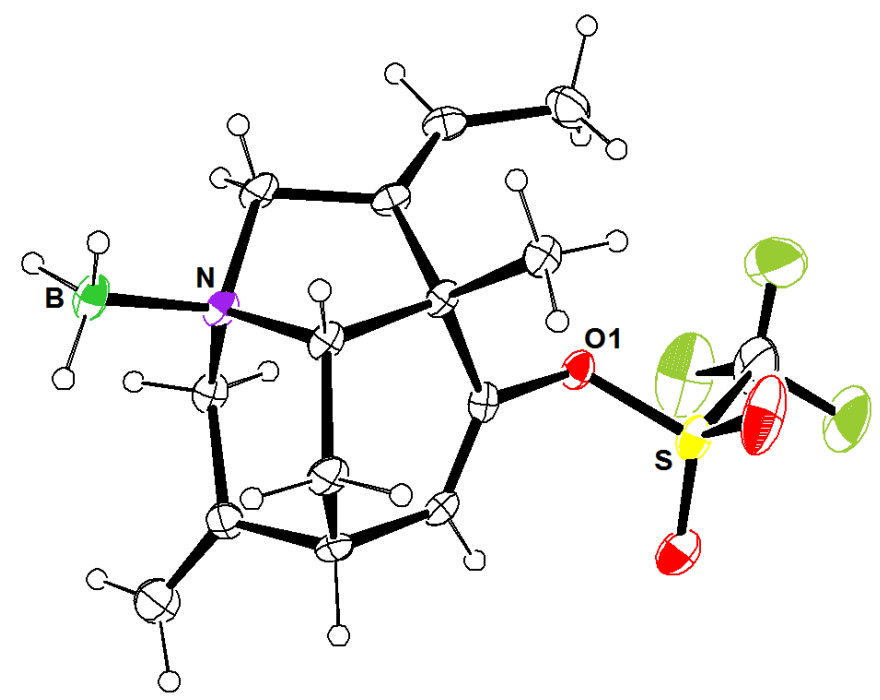

\begin{tabular}{|c|c|}
\hline Identification code & NBtriflate \\
\hline Empirical formula & $\mathrm{C}_{15} \mathrm{H}_{21} \mathrm{BF}_{3} \mathrm{NO}_{3} \mathrm{~S}$ \\
\hline Formula weight & 363.20 \\
\hline Temperature/K & 113.15 \\
\hline Crystal system & monoclinic \\
\hline Space group & $\mathrm{P} 21_{1}$ \\
\hline $\mathrm{a} / \AA$ & $8.2637(17)$ \\
\hline $\mathrm{b} / \AA$ & $10.027(2)$ \\
\hline $\mathrm{c} / \AA$ & $11.003(2)$ \\
\hline$\alpha /^{\circ}$ & 90 \\
\hline$\beta /{ }^{\circ}$ & $105.16(3)$ \\
\hline$\gamma /{ }^{\circ}$ & 90 \\
\hline Volume $/ \AA^{3}$ & $880.1(3)$ \\
\hline $\mathrm{Z}$ & 2 \\
\hline$\rho_{\text {calcg }} / \mathrm{cm}^{3}$ & 1.371 \\
\hline$\mu / \mathrm{mm}^{-1}$ & 0.226 \\
\hline $\mathrm{F}(000)$ & 380.0 \\
\hline Crystal size $/ \mathrm{mm}^{3}$ & $0.2 \times 0.18 \times 0.12$ \\
\hline Radiation & $\operatorname{MoK} \alpha(\lambda=0.71073)$ \\
\hline $2 \Theta$ range for data collection $/{ }^{\circ}$ & 5.108 to 55.96 \\
\hline Index ranges & $-10 \leq \mathrm{h} \leq 10,-13 \leq \mathrm{k} \leq 13,-14 \leq 1 \leq 14$ \\
\hline Reflections collected & 10022 \\
\hline Independent reflections & $4136\left[R_{\text {int }}=0.0765, R_{\text {sigma }}=0.1298\right]$ \\
\hline Data/restraints/parameters & $4136 / 25 / 220$ \\
\hline Goodness-of-fit on $\mathrm{F}^{2}$ & 0.883 \\
\hline Final $\mathrm{R}$ indexes $[\mathrm{I}>=2 \sigma(\mathrm{I})]$ & $\mathrm{R}_{1}=0.0603, \mathrm{wR}_{2}=0.1334$ \\
\hline Final $\mathrm{R}$ indexes [all data] & $\mathrm{R}_{1}=0.0834, \mathrm{wR}_{2}=0.1418$ \\
\hline Largest diff. peak/hole / e $\AA^{-3}$ & $0.41 /-0.40$ \\
\hline Flack parameter & 0.5 \\
\hline
\end{tabular}


ORTEP Drawing and Crystallographic Data of Compound 25 (CCDC 1899997)

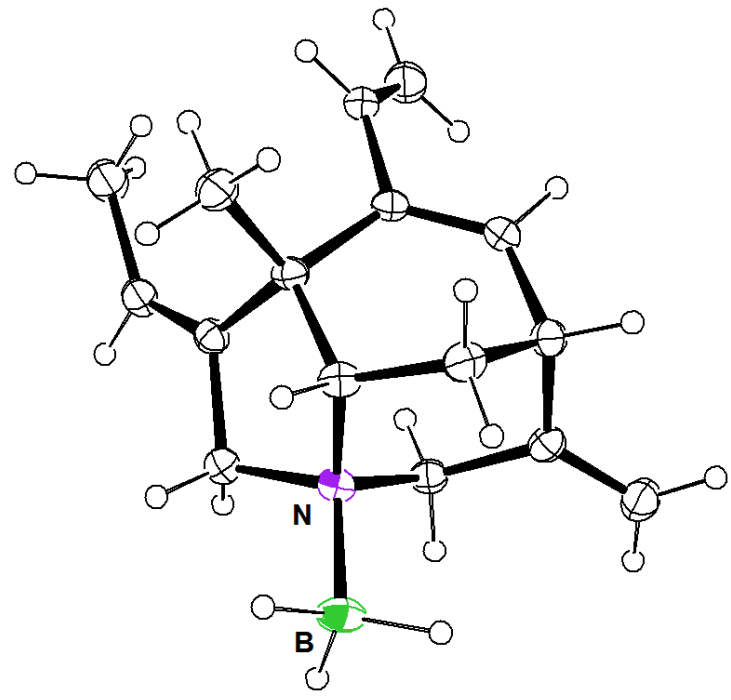

\begin{tabular}{|c|c|}
\hline Identification code & vinylcoupling \\
\hline Empirical formula & $\mathrm{C}_{16} \mathrm{H}_{24} \mathrm{BN}$ \\
\hline Formula weight & 241.17 \\
\hline Temperature/K & 113.15 \\
\hline Crystal system & orthorhombic \\
\hline Space group & $\mathrm{P} 2{ }_{1} 2_{1} 2_{1}$ \\
\hline $\mathrm{a} / \AA$ & $6.8133(14)$ \\
\hline $\mathrm{b} / \AA$ & $12.260(3)$ \\
\hline $\mathrm{c} / \AA$ & $17.431(4)$ \\
\hline$\alpha /^{\circ}$ & 90 \\
\hline$\beta /{ }^{\circ}$ & 90 \\
\hline$\gamma /{ }^{\circ}$ & 90 \\
\hline Volume $/ \AA^{3}$ & $1456.0(5)$ \\
\hline Z & 4 \\
\hline$\rho_{\text {calc }} \mathrm{g} / \mathrm{cm}^{3}$ & 1.100 \\
\hline$\mu / \mathrm{mm}^{-1}$ & 0.062 \\
\hline $\mathrm{F}(000)$ & 528.0 \\
\hline Crystal size $/ \mathrm{mm}^{3}$ & $0.2 \times 0.18 \times 0.12$ \\
\hline Radiation & $\operatorname{MoK} \alpha(\lambda=0.71073)$ \\
\hline $2 \Theta$ range for data collection $/{ }^{\circ}$ & 4.062 to 55.72 \\
\hline Index ranges & $-8 \leq \mathrm{h} \leq 8,-16 \leq \mathrm{k} \leq 16,-22 \leq 1 \leq 22$ \\
\hline Reflections collected & 17618 \\
\hline Independent reflections & $3460\left[\mathrm{R}_{\mathrm{int}}=0.0522, \mathrm{R}_{\text {sigma }}=0.0413\right]$ \\
\hline Data/restraints/parameters & $3460 / 3 / 174$ \\
\hline Goodness-of-fit on $\mathrm{F}^{2}$ & 1.059 \\
\hline Final $\mathrm{R}$ indexes $[\mathrm{I}>=2 \sigma(\mathrm{I})]$ & $\mathrm{R}_{1}=0.0480, \mathrm{wR}_{2}=0.1075$ \\
\hline Final $\mathrm{R}$ indexes [all data] & $\mathrm{R}_{1}=0.0536, \mathrm{wR}_{2}=0.1115$ \\
\hline Largest diff. peak/hole / e $\AA^{-3}$ & $0.14 /-0.19$ \\
\hline Flack parameter & 0.5 \\
\hline
\end{tabular}


ORTEP Drawing and Crystallographic Data of Compound 26 (CCDC 1893592)

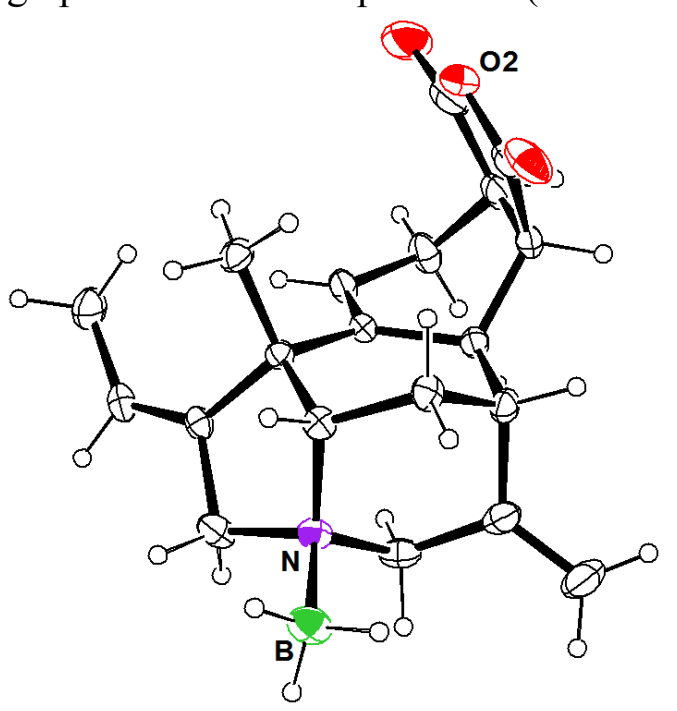

\begin{tabular}{|c|c|}
\hline Identification code & DielsAlderNBH3 \\
\hline Empirical formula & $\mathrm{C}_{20} \mathrm{H}_{26} \mathrm{BNO}_{3}$ \\
\hline Formula weight & 339.23 \\
\hline Temperature/K & 113.15 \\
\hline Crystal system & orthorhombic \\
\hline Space group & $\mathrm{P} 2{ }_{1} 2{ }_{1}{ }_{1}$ \\
\hline $\mathrm{a} / \AA ̊$ & $9.3851(19)$ \\
\hline $\mathrm{b} / \AA$ & $11.151(2)$ \\
\hline $\mathrm{c} / \AA ̊$ & $17.097(3)$ \\
\hline$\alpha /^{\circ}$ & 90 \\
\hline$\beta /{ }^{\circ}$ & 90 \\
\hline$\gamma /{ }^{\circ}$ & 90 \\
\hline Volume $/ \AA^{3}$ & $1789.3(6)$ \\
\hline $\mathrm{Z}$ & 4 \\
\hline$\rho_{\text {calc }} \mathrm{g} / \mathrm{cm}^{3}$ & 1.259 \\
\hline$\mu / \mathrm{mm}^{-1}$ & 0.083 \\
\hline $\mathrm{F}(000)$ & 728.0 \\
\hline Crystal size $/ \mathrm{mm}^{3}$ & $0.2 \times 0.18 \times 0.12$ \\
\hline Radiation & $\operatorname{MoK} \alpha(\lambda=0.71073)$ \\
\hline $2 \Theta$ range for data collection $/{ }^{\circ}$ & 4.36 to 55.754 \\
\hline Index ranges & $-12 \leq \mathrm{h} \leq 11,-14 \leq \mathrm{k} \leq 14,-22 \leq 1 \leq 22$ \\
\hline Reflections collected & 21669 \\
\hline Independent reflections & $4277\left[\mathrm{R}_{\text {int }}=0.0522, \mathrm{R}_{\text {sigma }}=0.0414\right]$ \\
\hline Data/restraints/parameters & $4277 / 0 / 230$ \\
\hline Goodness-of-fit on $\mathrm{F}^{2}$ & 1.077 \\
\hline Final $\mathrm{R}$ indexes $[\mathrm{I}>=2 \sigma(\mathrm{I})]$ & $\mathrm{R}_{1}=0.0545, \mathrm{wR}_{2}=0.1375$ \\
\hline Final R indexes [all data] & $\mathrm{R}_{1}=0.0602, \mathrm{wR}_{2}=0.1423$ \\
\hline Largest diff. peak/hole / e $\AA^{-3}$ & $0.24 /-0.23$ \\
\hline Flack parameter & 0.5 \\
\hline
\end{tabular}


ORTEP Drawing and Crystallographic Data of Compound 27 (CCDC 1893593)

\begin{tabular}{|c|c|}
\hline Identification code & DielsAlderNBH2Br \\
\hline Empirical formula & $\mathrm{C}_{20} \mathrm{H}_{23} \mathrm{BBrNO}_{3}$ \\
\hline Formula weight & 416.11 \\
\hline Temperature/K & $179.98(18)$ \\
\hline Crystal system & monoclinic \\
\hline Space group & $\mathrm{P} 2{ }_{1}$ \\
\hline $\mathrm{a} / \AA$ & $10.0780(2)$ \\
\hline $\mathrm{b} / \AA$ & $12.7337(3)$ \\
\hline $\mathrm{c} / \AA$ & $14.7167(4)$ \\
\hline$\alpha /^{\circ}$ & 90 \\
\hline$\beta /{ }^{\circ}$ & $91.616(2)$ \\
\hline$\gamma /{ }^{\circ}$ & 90 \\
\hline Volume $/ \AA^{3}$ & $1887.85(8)$ \\
\hline $\mathrm{Z}$ & 4 \\
\hline$\rho_{\text {calc }} \mathrm{g} / \mathrm{cm}^{3}$ & 1.464 \\
\hline$\mu / \mathrm{mm}^{-1}$ & 2.197 \\
\hline $\mathrm{F}(000)$ & 856.0 \\
\hline Crystal size $/ \mathrm{mm}^{3}$ & $0.1 \times 0.1 \times 0.1$ \\
\hline Radiation & $\operatorname{MoK} \alpha(\lambda=0.71073)$ \\
\hline $2 \Theta$ range for data collection $/{ }^{\circ}$ & 4.044 to 50.05 \\
\hline Index ranges & $-10 \leq \mathrm{h} \leq 11,-15 \leq \mathrm{k} \leq 15,-17 \leq 1 \leq 17$ \\
\hline Reflections collected & 20929 \\
\hline Independent reflections & $6560\left[\mathrm{R}_{\text {int }}=0.0225, \mathrm{R}_{\text {sigma }}=0.0242\right]$ \\
\hline Data/restraints/parameters & $6560 / 1 / 473$ \\
\hline Goodness-of-fit on $\mathrm{F}^{2}$ & 1.031 \\
\hline Final $\mathrm{R}$ indexes $[\mathrm{I}>=2 \sigma(\mathrm{I})]$ & $\mathrm{R}_{1}=0.0403, \mathrm{wR}_{2}=0.1123$ \\
\hline Final $\mathrm{R}$ indexes [all data] & $\mathrm{R}_{1}=0.0440, \mathrm{wR}_{2}=0.1153$ \\
\hline Largest diff. peak/hole / e $\AA^{-3}$ & $0.86 /-0.94$ \\
\hline Flack parameter & $0.027(3)$ \\
\hline
\end{tabular}


ORTEP Drawing and Crystallographic Data of Compound 30 (CCDC 1899998)

\begin{tabular}{|c|c|}
\hline Identification code & AreneE \\
\hline Empirical formula & $\mathrm{C}_{22} \mathrm{H}_{28} \mathrm{BNO}_{4}$ \\
\hline Formula weight & 381.26 \\
\hline Temperature/K & 113.15 \\
\hline Crystal system & triclinic \\
\hline Space group & $\mathrm{P} 1$ \\
\hline $\mathrm{a} / \AA$ & $5.9667(12)$ \\
\hline $\mathrm{b} / \AA$ & $8.5345(17)$ \\
\hline $\mathrm{c} / \AA \AA$ & $10.417(2)$ \\
\hline$\alpha /{ }^{\circ}$ & $87.38(3)$ \\
\hline$\beta /{ }^{\circ}$ & $87.11(3)$ \\
\hline$\gamma /{ }^{\circ}$ & $80.44(3)$ \\
\hline Volume $/ \AA^{3}$ & $522.08(19)$ \\
\hline $\mathrm{Z}$ & 1 \\
\hline$\rho_{\text {calc }} \mathrm{g} / \mathrm{cm}^{3}$ & 1.213 \\
\hline$\mu / \mathrm{mm}^{-1}$ & 0.082 \\
\hline $\mathrm{F}(000)$ & 204.0 \\
\hline Crystal size $/ \mathrm{mm}^{3}$ & $0.2 \times 0.18 \times 0.12$ \\
\hline Radiation & $\operatorname{MoK} \alpha(\lambda=0.71073)$ \\
\hline $2 \Theta$ range for data collection $/{ }^{\circ}$ & 6.114 to 50.042 \\
\hline Index ranges & $-7 \leq \mathrm{h} \leq 7,-10 \leq \mathrm{k} \leq 10,-12 \leq 1 \leq 12$ \\
\hline Reflections collected & 4861 \\
\hline Independent reflections & $3369\left[\mathrm{R}_{\mathrm{int}}=0.0605, \mathrm{R}_{\text {sigma }}=0.1412\right]$ \\
\hline Data/restraints/parameters & $3369 / 6 / 267$ \\
\hline Goodness-of-fit on $\mathrm{F}^{2}$ & 0.841 \\
\hline Final $R$ indexes $[\mathrm{I}>=2 \sigma(\mathrm{I})]$ & $\mathrm{R}_{1}=0.0511, \mathrm{wR}_{2}=0.1226$ \\
\hline Final $\mathrm{R}$ indexes [all data] & $\mathrm{R}_{1}=0.0682, \mathrm{wR}_{2}=0.1548$ \\
\hline Largest diff. peak/hole / e $\AA^{-3}$ & $0.33 /-0.33$ \\
\hline Flack parameter & 0.5 \\
\hline
\end{tabular}

